\title{
On the Relativistic Canonical Quantum Mechanics and Field Theory of Arbitrary Spin
}

\author{
V.M. Simulik \\ Institute of Electron Physics, National Academy of Sciences, Uzhgorod, 88000, Ukraine
}

Copyright (c) 2017 by authors, all rights reserved. Authors agree that this article remains permanently open access under the terms of the Creative Commons Attribution License 4.0 International License

\begin{abstract}
The new relativistic equations of motion for the particles with arbitrary spin and nonzero mass, suggested by author in years 2014-2016, are under consideration. The complete version of brief paper in J. Phys: Conf. Ser., 670 (2016) 012047(1-16) is given. The axiomatic level description of the relativistic canonical quantum mechanics of an arbitrary mass and spin has been given. The 64-dimensional $\mathrm{Cl}^{\mathrm{R}}(0,6)$ algebra in terms of Dirac gamma matrices has been suggested. The interpretation of the 28-dimensional gamma matrix representation of $\mathrm{SO}(8)$ algebra over the field of real numbers is given. The link between the relativistic canonical quantum mechanics of the arbitrary spin and the covariant local field theory in the form of extended Foldy-Wouthuysen transformation has been found. Different methods of the Dirac equation derivation have been reviewed. The manifestly covariant field equation for an arbitrary spin, that follows from the corresponding equation of relativistic canonical quantum mechanics, has been considered. The found equations are without redundant components. The partial examples for spin $s=3 / 2$ and $s=2$ are presented. The covariant local field theory equations for spin $\mathrm{s}=(3 / 2,3 / 2)$ particle-antiparticle doublet and spin $\mathrm{s}=(2,2)$ particle-antiparticle doublet have been introduced. The Maxwell and slightly generalized Maxwell-like equations containing mass member have been considered as well.
\end{abstract}

Keywords Relativistic Quantum Mechanics, Quantum Field Theory, Relativistic Wave Equations, Higher Spin, Arbitrary Spin, Foldy-Wouthuysen Representation, Applied Clifford Algebras

\section{Introduction}

Recently in [1] the interesting results in the area of relativistic quantum mechanics and quantum field theory have been presented. Here in this article this investigation has been continued by the general forms of the partial cases visualized in [1]. The brief version was given already in [2, 3]. Consider the more complete version.

Thus, here the general forms of quantum-mechanical and covariant equations for arbitrary spin together with the general description of the arbitrary spin field formalism are presented. The corresponding relativistic quantum mechanics of arbitrary spin is given as the system of axioms. The partial cases of the spin $\mathrm{s}=(0,0)$ and spin $s=(3 / 2,3 / 2)$ particle-antiparticle doublets are considered in explicit forms. The review of the different investigations in the area of relativistic canonical quantum mechanics (RCQM) is given and the brief analysis of the existing approaches to the field theory of arbitrary spin is initiated.

Note that in the Dirac model $[4,5]$ the quantummechanical interpretation is not evident. It has been demonstrated in $[1,6,7]$ that the quantum-mechanical interpretation is much more clear in the Foldy-Wouthuysen (FW) model $[6,7]$. Nevertheless, the complete quantummechanical picture is possible only in the framework of RCQM. This assertion is one of the main conclusions proved in [1].

The relativistic quantum mechanics under consideration is called canonical due to three main reasons. (i) The model under consideration has direct link with nonrelativistic quantum mechanics based on nonrelativistic Schrödinger equation. The principles of heredity and correspondence with other models of physical reality leads directly to nonrelativistic Schrödinger quantum mechanics. (ii) The FW model is already called by many authors as the canonical representation of the Dirac equation or a canonical field model, see, e. g., the paper [7]. And the difference between the field model given by FW and the RCQM is minimal in corresponding equations it is only the presence and absence of beta matrix. (iii) The list of relativistic quantummechanical models is long. The Dirac model and the FW model are called by the "old" physicists as the relativistic 
quantum mechanics as well (one of my tasks in this paper is to show in visual and demonstrative way that these models have only weak quantum-mechanical interpretation). Further, the fractional relativistic quantum mechanics and the proper-time relativistic quantum mechanics can be listed (recall matrix formulation by W. Heisenberg, Feynman's sum over path's quantum theory, many-worlds interpretation by H. Everett), etc. Therefore, in order to avoid a confusion the model under consideration must have its proper name. Due to the reasons (i)-(iii) the best name for it is RCQM.

The general and fundamental goals here are as follows: (i) visual and demonstrative generalization of existing RCQM for the case of arbitrary spin, (ii) more complete formulation of this model on axiomatic level (on the test example of spin $s=(1 / 2,1 / 2)$ particle-antiparticle doublet), (iii) vertical and horizontal links between the three different models of physical reality: relativistic quantum mechanics of arbitrary spin in canonical form, canonical (FW type) field theory of any spin, locally covariant (Dirac and Maxwell type) field theory of arbitrary spin.

The concepts, definitions and notations here are the same as in [1]. For example, in the Minkowski space-time

$$
\begin{gathered}
\mathrm{M}(1,3)=\left\{x \equiv\left(x^{\mu}\right)=\left(x^{0}=t, \vec{x} \equiv\left(x^{j}\right)\right)\right\} ; \\
\mu=\overline{0,3}, j=1,2,3,
\end{gathered}
$$

$x^{\mu}$ denotes the Cartesian (covariant) coordinates of the points of the physical space-time in the arbitrary-fixed inertial reference frame (IRF). We use the system of units with $\hbar=c=1$. The metric tensor is given by

$$
\begin{gathered}
g^{\mu \nu}=g_{\mu \nu}=g_{\nu}^{\mu},\left(g_{\nu}^{\mu}\right)=\operatorname{diag}(1,-1,-1,-1) ; \\
x_{\mu}=g_{\mu \nu} x^{\mu},
\end{gathered}
$$

and summation over the twice repeated indices is implied.

\section{Dirac's comment}

Note that the square-root operator equation, which is the main equation of RCQM, has been rejected by Dirac. In his consideration in [5] (chapter 11, section 67) of the main steps of [4] Dirac discussed this equation. His comment was as follows.

Let us consider first the case of the motion of an electron in the absence of an electromagnetic field, so that the problem is simply that of the free particle, as dealt with in $\S 30$, with the possible addition of internal degrees of freedom. The relativistic Hamiltonian provided by classical mechanics for this system is given by equation 23 of $\S 30$, and leads to the wave equation

$$
\left\{p_{0}-\left(m^{2} c^{2}+p_{1}^{2}+p_{2}^{2}+p_{3}^{2}\right)^{\frac{1}{2}}\right\} \psi=0,
$$

where the p's are interpreted as operators in accordance with (4). Equation (5), although it takes into account the relation between energy and momentum required by relativity, is yet unsatisfactory from the point of view of relativistic theory, because it is very unsymmetrical between $p_{0}$ and the other p's, so much so that one cannot generalize it in a relativistic way to the case when there is a field present. We must therefore look for a new wave equation.

Today another reason of Dirac's rejection of the squareroot operator equation is evident as well. The operations with the Dirac's Hamiltonian are too much easier than the operations with the pseudo-differential Hamiltonian of the equation

$$
i \partial_{t} f(x)=\sqrt{m^{2}-\Delta} f(x)
$$

for the $\mathrm{N}$-component wave function

$$
f \equiv \operatorname{column}\left(f^{1}, f^{2}, \ldots, f^{\mathrm{N}}\right), \quad \mathrm{N}=2 s+1, \quad f \in \mathrm{H}^{3, \mathrm{~N}},
$$

in the case of particle singlet and for the M-component wave function $(\mathrm{M}=2 \mathrm{~N}=2(2 s+1))$ in the case of particle-antiparticle doublet. Here $\mathrm{H}^{3, \mathrm{~N}}$ is the Hilbert space of N-component functions. Note that namely (3) is the equation of motion in the RCQM of arbitrary spin, see [1] for the details.

Nevertheless, today, contrary to the year 1928, the definition of the pseudo-differential (non-local) operator

$$
\begin{gathered}
\widehat{\omega} \equiv \sqrt{\widehat{\vec{p}}^{2}+m^{2}}=\sqrt{-\Delta+m^{2}} \geq m>0, \\
\widehat{\vec{p}} \equiv\left(\widehat{p}^{j}\right)=-i \nabla, \quad \nabla \equiv\left(\partial_{\ell}\right),
\end{gathered}
$$

is well known. The action of the operator (5) in the coordinate representation (see, e. g. [8]) is given by

$$
\widehat{\omega} f(t, \vec{x})=\int d^{3} y K(\vec{x}-\vec{y}) f(t, \vec{y}),
$$

where the function $K(\vec{x}-\vec{y})$ has the form $K(\vec{x}-$ $\vec{y})=-\frac{2 m^{2} K_{2}(m|\vec{x}-\vec{y}|)}{(2 \pi)^{2}|\vec{x}-\vec{y}|^{2}}$ and $K_{\nu}(z)$ is the modified Bessel function (Macdonald function), $|\vec{a}|$ designates the norm of the vector $\vec{a}$.

Further, the following integral form

$$
\begin{gathered}
(\widehat{\omega} f)(t, \vec{x})=\frac{1}{(2 \pi)^{\frac{3}{2}}} \int d^{3} k e^{i \vec{k} \vec{x}} \widetilde{\omega} \widetilde{f}(t, \vec{k}), \\
\widetilde{\omega} \equiv \sqrt{\vec{k}^{2}+m^{2}}, \quad \widetilde{f} \in \widetilde{\mathrm{H}}^{3, \mathrm{~N}},
\end{gathered}
$$

of the operator $\widehat{\omega}$ is used often, see, e. g., [7, 9], where $f$ and $\widetilde{f}$ are linked by the 3 -dimensional Fourier transformations

$$
\begin{aligned}
& f(t, \vec{x})=\frac{1}{(2 \pi)^{\frac{3}{2}}} \int d^{3} k e^{i \vec{k} \vec{x}} \tilde{f}(t, \vec{k}) \Leftrightarrow \\
& \tilde{f}(t, \vec{k})=\frac{1}{(2 \pi)^{\frac{3}{2}}} \int d^{3} k e^{-i \vec{k} \vec{x}} \tilde{f}(t, \vec{x}),
\end{aligned}
$$

(in (8) $\vec{k}$ belongs to the spectrum $\mathrm{R}_{\vec{k}}^{3}$ of the operator $\widehat{\vec{p}}$, and the parameter $t \in(-\infty, \infty) \subset \mathrm{M}(1,3))$. 
Note that the space of states $\mathrm{H}^{3, \mathrm{~N}}$ is invariant with respect to the Fourier transformation (8). Therefore, both $\vec{x}$ realization $\mathrm{H}^{3, \mathrm{~N}}$ and $\vec{k}$-realization $\widetilde{\mathrm{H}}^{3, \mathrm{~N}}$ of the space of states are suitable for the purposes of our consideration. In the $\vec{k}$-realization the Schrödinger-Foldy equation has the algebraic-differential form

$i \partial_{t} \tilde{f}(t, \vec{k})=\sqrt{\vec{k}^{2}+m^{2}} \widetilde{f}(t, \vec{k}) ; \quad \vec{k} \in \mathrm{R}_{\vec{k}}^{3}, \quad \tilde{f} \in \widetilde{\mathrm{H}}^{3, \mathrm{~N}}$

Below in the places, where misunderstanding is impossible, the symbol "tilde" is omitted.

Thus, today on the basis of above given definitions the difficulties, which stopped Dirac in 1928, can be overcome.

\section{Foldy's contribution}

The name of the person, whose contribution in the theoretical model based on the equation (3) was decisive, is Leslie Lawrance Foldy (1919-2001).

His interesting biography is presented in [10]. Les was born in Sabinov, Czechoslovakia, on 26 October 1919, into a family with Hungarian roots. His parents named him Laszlo Földi. In the turbulent times following World War I, he immigrated with his parents to the US in 1921. His father changed the family's last name and Les's first name; Les later added his middle name, unaware of its more common spellings.

The first step of Foldy's contribution is visualization of the quantum mechanical interpretation of the Dirac equation on the basis of transformation to the canonical (quantum-mechanical) representation [6]. This transformation was suggested together with the Netherlander Siegfried (Sieg) Wouthuysen (pronounced Vout-high-sen). In this FW representation of the Dirac equation the quantummechanical interpretation is much more clear. Nevertheless, the direct and evident quantum-mechanical interpretation of the spin $s=(1 / 2,1 / 2)$ particle-antiparticle doublet can be fulfilled only within the framework of the RCQM: the start was given in [7], see also the consideration in [1].

In our investigations we always marked the role of $\mathrm{L}$. Foldy. Taking into account the L. Foldy's contribution in the construction of RCQM and his proof of the principle of correspondence between RCQM and non-relativistic quantum mechanics, we propose [11, 12] and [1] to call the $N$-component equation (3) as the Schrödinger-Foldy equation. Note here that equation (3), which is a direct sum of one component spinless Salpeter equations [13], has been introduced in the formula (21) of [7]. Furthermore, note here that the nonlocal Poincaré group representation generators are known from the formulas (B-25)-(B-28) of the L. Foldy's paper [7].

\section{Brief review of the relativistic ca- nonical quantum mechanics status quo}

Contrary to the times of papers $[4,6,7,13]$, the RCQM today is enough approbated and generally accepted theory. The spinless Salpeter equation has been introduced in [13]. The allusion on the RCQM and the first steps are given in [7], where the Salpeter equation for the 2s+1-component wave function was considered and the cases of $s=1 / 2, s=1$ were presented as an examples. In [14] L. Foldy continued his investigations [7] by the consideration of the relativistic particle systems with interaction. The interaction was introduced by the specific group-theoretical method.

After that in the RCQM were developed both the construction of mathematical foundations and the solution of concrete quantum-mechanical problems for different potentials. Some mathematical foundations and spectral theory of pseudo-differential operator $\sqrt{\vec{p}^{2}+m^{2}}-Z e^{2} / r$ were given in [15-18]. The application of the RCQM to the quark-antiquark bound state problem can be found in $[19,20]$. The numerical solutions of the RCQM equation for arbitrary confining potentials were presented in [20]. In [21] the spinless Salpeter equation for the $\mathrm{N}$ particle system of spinless bosons in gravitational interaction was applied. In [22] a lower bound on the maximum mass of a boson star on the basis of the Hamiltonian $\sqrt{\vec{p}^{2}+m^{2}}-\alpha / r$ has been calculated. In [23] results calculated by the author with the spinless Salpeter equation are compared with those obtained from Schrodinger's equation for heavy-quark systems, heavy-light systems, and light-quark systems. In each case the Salpeter energies agree with experiment substantially better than the Schrodinger energies. The paper [24] deal with an investigation of the exact numerical solutions. The spinless Salpeter equation with the Coulomb potential is solved exactly in momentum space and is shown to agree very well with a coordinate-space calculation. In $[25,26]$ the problem of spectrum of energy eigenvalues calculations on the basis of the spinless Salpeter equation is considered. The spinless relativistic Coulomb problem is studied. It was shown how to calculate, by some special choices of basis vectors in the Hilbert space of solutions, for the rather large class of power-law potentials, at least upper bounds on these energy eigenvalues. The authors of $[25,26]$ proved that for the lowest-lying levels, this may be done even analytically. In the paper [27] the spinless Salpeter equation was rewritten into integral and integro-differential equations. Some analytical results concerning the spinless Salpeter equation and the action of the square-root operator have been presented. Further, in [28] F. Brau constructed an analytical solution of the one-dimensional spinless Salpeter equation with a Coulomb potential supplemented by a hard core interaction, which keeps the particle in the $x$ positive region. In the context of RCQM based on the spinless Salpeter equation it was shown [29] how to construct a large 
class of upper limits on the critical value, $g_{c}^{(\ell)}$, of the coupling constant, $g$, of the central potential, $V(r)=-g v(r)$. In [30] a lower bounds on the ground state energy, in one and three dimensions, for the spinless Salpeter equation applicable to potentials, for which the attractive parts are in $\mathrm{L}^{\mathrm{p}}\left(\mathrm{R}^{\mathrm{n}}\right)$ for some $\mathrm{p} i \mathrm{n}(\mathrm{n}=1$ or 3$)$, are found. An extension to confining potentials, which are not in $\mathrm{L}^{\mathrm{p}}\left(\mathrm{R}^{\mathrm{n}}\right)$, is also presented. In the paper [31], the authors used the theory of fractional powers of linear operators to construct a general (analytic) representation theory for the square-root energy operator $\gamma^{0} \sqrt{\vec{p}^{2}+m^{2}}+V$ of FW canonical field theory, which is valid for all values of the spin. The example of the spin 1/2 case, considering a few simple yet solvable and physically interesting cases, is presented in details in order to understand how to interpret the operator. Note that corresponding results for the RCQM can be found from the FW canonical field theory results [31] with the help of our transformation (see the section 9 in [1]). Using the momentum space representation, the authors of [32] presented an analytical treatment of the one-dimensional spinless Salpeter equation with a Coulomb interaction. The exact bound-state energy equation was determined. The results obtained were shown to agree very well with exact numerical calculations existing in the literature. In [33] an exact analytical treatment of the spinless Salpeter equation with a one dimensional Coulomb interaction in the context of quantum mechanics with modified Heisenberg algebra implying the existence of a minimal length was presented. The problem was tackled in the momentum space representation. The bound-state energy equation and the corresponding wave functions were exactly obtained. The probability current for a quantum spinless relativistic particle was introduced [8] based on the Hamiltonian dynamics approach using the spinless Salpeter equation. The correctness of the presented formalism was illustrated by examples of exact solutions to the spinless Salpeter equation including the new ones. Thus, in [8] the partial wave packet solutions of this equation have been presented: the solutions for free massless and massive particle on a line, for massless particle in a linear potential, plane wave solution for a free particle (these solution is given here in formula (8) for N-component case), the solution for free massless particle in three dimensions. Further, in the paper [34] other time dependent wave packet solutions of the free spinless Salpeter equation are given. Taking into account the relation of such wave packets to the Lévy process the spinless Salpeter equation (in one dimensional space-time) is called in [34] as the Lévy-Schrödinger equation. The several examples of the characteristic behavior of such wave packets have been shown, in particular of the multimodality arising in their evolutions: a feature at variance with the typical diffusive unimodality of both the corresponding Lévy process densities and usual Schrödinger wave functions. A generic upper bound is obtained [35] for the spinless Salpeter equation with two different masses. Analytical results are presented for systems relevant for hadronic physics: Cou- lomb and linear potentials when a mass is vanishing. A detailed study for the classical and the quantum motion of a relativistic massless particle in an inverse square potential has been presented recently in [36]. The quantum approach to the problem was based on the exact solution of the corresponding spinless Salpeter equation for bound states. Finally, in [36] the connection between the classical and the quantum descriptions via the comparison of the associated probability densities for momentum has been made. The goal of the recent paper [37] is a comprehensive analysis of the intimate relationship between jump-type stochastic processes (e. g. Lévy flights) and nonlocal (due to integrodifferential operators involved) quantum dynamics. In [37] a special attention is paid to the spinless Salpeter (here, $m \geq 0$ ) equation and the evolution of various wave packets, in particular to their radial expansion in 3D. Foldy's synthesis of covariant particle equations is extended to encompass free Maxwell theory, which however is devoid of any particle content. Links with the photon wave mechanics are explored. The authors of [37] takes into account our results [11] presented also in more earlier preprint, see the last reference in [37].

In the papers $[11,12]$, where we started our investigations in RCQM, this relativistic model for the test case of the spin $s=(1 / 2,1 / 2)$ particle-antiparticle doublet is formulated. In [11], this model is considered as the system of the axioms on the level of the von Neumann monograph [38], where the mathematically well-defined consideration of the nonrelativistic quantum mechanics was given. Furthermore, in $[11,12]$ the operator link between the spin $s=(1 / 2,1 / 2)$ particle-antiparticle doublet RCQM and the Dirac theory is given and Foldy's synthesis of covariant particle equations is extended to the start from the RCQM of the spin $s=(1 / 2,1 / 2)$ particle-antiparticle doublet. In [1] the same procedure is fulfilled for the spin $\mathrm{s}=(1,1), \mathrm{s}=(1,0,1,0), \mathrm{s}=(3 / 2,3 / 2), \mathrm{s}=(2,2), \mathrm{s}=(2,0,2,0)$ and spin $s=(2,1,2,1)$ RCQM. The corresponding equations, which follow from the RCQM for the covariant local field theory, are introduced.

Therefore, here and in [1-3] we are not going to formulate a new relativistic quantum mechanics! The foundations of RCQM based on the spinless Salpeter equation are already formulated in [7, 8, 11-37].

\section{Brief analysis of the covariant equations for an arbitrary spin}

One of the goals of [1-3] (and this article as well) is the link between the RCQM of an arbitrary spin and the different approaches to the covariant local field theory of an arbitrary spin. Surely, at least the brief analysis of the existing covariant equations for an arbitrary spin should be presented.

Note that in [1-3] and here only the first-order particle and the field equations (together with their canonical nonlo- 
cal pseudo-differential representations) are considered. The second order equations (like the Klein-Gordon-Fock equation) are not the subject of this investigation. Thus, the Bhabha, Bargman-Wigner, Pauli-Fierz and many other such type equations are under consideration.

Different approaches to the description of the field theory of an arbitrary spin can be found in [7,39-56]. Here and in [1-3] only the approach started in [7] is the basis for further application. Other results given in [39-56] are not used here.

Note only some general deficiencies of the known equations for arbitrary spin. The consideration of the partial cases, when the substitution of the fixed value of spin is fulfilled, is not successful in all cases. For example, for the spin $s>1$ existing equations have the redundant components and should be complemented by some additional conditions. Indeed, the known equations $[40,41,57]$ for the spin $\mathrm{s}=3 / 2$ (and their confirmation in [58]) should be essentially complemented by the additional conditions. The main difficulty in the models of an arbitrary spin is the interaction between the fields of higher-spin. Even the quantization of higher-spin fields generated the questions. These and other deficiencies of the known equations for higher-spin are considered in [59-77] (a brief review of deficiencies see in [69]).

Equations suggested in [1-3] and here are free of these deficiencies. The start of such consideration is taken from [7], where the main foundations of the RCQM are formulated. In the texts of [1-3] and here the results of [7] are generalized and extended. The operator link between the results of [6] and [7] (between the canonical FW type field theory and the RCQM) is suggested. Note that the cases $s=3 / 2$ and $s=2$ are not presented in [7], especially in explicit demonstrative forms. The results of [1-3] formally look like as the given in $[43,45]$. Nevertheless, the difference is evident. Indeed, in $[43,45]$ the Bargman-Wigner equations and their Foldy-Wouthuysen representations have been considered. Our approach [1-3] is completely different in comparison with Bargman-Wigner approach.

Equations suggested in [1-3] do not contain redundant components. The equations from [1-3] have been derived directly from the well-defined equations of relativistic canonical quantum mechanics, where every component is well-defined. Therefore, the partial differential equations for arbitrary spin found in [1-3] are without redundant components. It is the advantage of equations from [1-3] in comparison with equations considered in [39-56].

Indeed, the Rarita-Schwinger equation for spin $s=3 / 2$ has 16 components, whereas our equation [1-3] for spin $s=(3 / 2,3 / 2)$ particle-antiparticle doublet has 8 components. The Bargman-Wigner equation [43, 45] in partial case $s=3 / 2$ has 12 components. Bhabha itself [78] have analyzed the partial case $s=3 / 2$ for his equation [42]. He have found [78] that in this case his equation [42] coincides with the Rarita-Schwinger equation, i.e. has 16 components. Therefore, in [1-3] the new equation for arbitrary spin has been suggested.
Even this brief analysis makes us sure in the prospects of the investigations started in [1-3]. The successful description of the arbitrary spin field models is not the solved problem today.

\section{Axioms of the relativistic canoni- cal quantum mechanics of an arbi- trary spin}

The RCQM of the arbitrary spin given in sections 2 and 18 of [1] can be formulated at the level of von Neumann's consideration [38]. The difference is only in relativistic invariance and in the consideration of multicomponent and multidimensional objects.

The partial case of axiomatic formulation is already given in section 7 of [1] at the example of spin $s=1 / 2$ particleantiparticle doublet. The RCQM of the arbitrary spin particle-antiparticle doublet (or particle singlet) can be formulated similarly as the corresponding generalization of this partial case.

Below the brief presentation of the list of the axioms is given. Note that some particular content of these axioms is already given in section 2 of [1], where the RCQM of the arbitrary spin particle singlet has been formulated.

\subsection{On the space of states}

The space of states of isolated arbitrary spin particle singlet in an arbitrarily-fixed inertial frame of reference (IFR) in its $\vec{x}$-realization is the Hilbert space

$$
\begin{gathered}
\mathrm{H}^{3, \mathrm{~N}}=\mathrm{L}_{2}\left(\mathrm{R}^{3}\right) \otimes \mathrm{C}^{\otimes \mathrm{N}}=\left\{f=\left(f^{\mathrm{N}}\right): \mathrm{R}^{3} \rightarrow \mathrm{C}^{\otimes \mathrm{N}} ;\right. \\
\left.\int d^{3} x|f(t, \vec{x})|^{2}<\infty\right\}, \quad \mathrm{N}=2 s+1,
\end{gathered}
$$

of complex-valued $\mathrm{N}$-component square-integrable functions of $x \in \mathrm{R}^{3} \subset \mathrm{M}(1,3)$ (similarly, in momentum, $\vec{p}$ realization). In (10) $d^{3} x$ is the Lebesgue measure in the space $\mathrm{R}^{3} \subset \mathrm{M}(1,3)$ of the eigenvalues of the position operator $\vec{x}$ of the Cartesian coordinate of the particle in an arbitrary-fixed IFR. Further, $\vec{x}$ and $\vec{p}$ are the operators of canonically conjugated dynamical variables of the spin $\mathrm{s}=(1 / 2,1 / 2)$ particle-antiparticle doublet, and the vectors $f$, $\tilde{f}$ in $\vec{x}$ - and $\vec{p}$-realizations are linked by the 3 -dimensional Fourier transformation (the variable $t$ is the parameter of time-evolution).

The mathematical correctness of the consideration demands the application of the rigged Hilbert space

$$
\mathrm{S}^{3, \mathrm{~N}} \equiv \mathrm{S}\left(\mathrm{R}^{3}\right) \times \mathrm{C}^{\mathrm{N}} \subset \mathrm{H}^{3, \mathrm{~N}} \subset \mathrm{S}^{3, \mathrm{~N} *} .
$$

where the Schwartz test function space $\mathrm{S}^{3, \mathrm{~N}}$ is the core (i. e., it is dense both in $\mathrm{H}^{3, \mathrm{~N}}$ and in the space $\mathrm{S}^{3, \mathrm{~N} *}$ of the $\mathrm{N}$-component Schwartz generalized functions). The space $\mathrm{S}^{3, \mathrm{~N} *}$ is conjugated to that of the Schwartz test functions $\mathrm{S}^{3, \mathrm{~N}}$ by the corresponding topology (see, e. g. [79]). 
Strictly speaking, the mathematical correctness of consideration demands to make the calculations in the space $\mathrm{S}^{3, \mathrm{~N} *}$ of generalized functions, i. e. with the application of cumbersome functional analysis (see, e. g. [80]). Nevertheless, one can take into account the properties of the Schwartz test function space $S^{3, N}$ in the triple (11). The space $S^{3, N}$ is dense both in quantum-mechanical space $\mathrm{H}^{3, \mathrm{~N}}$ and in the space of generalized functions $\mathrm{S}^{3, \mathrm{~N} *}$. Therefore, any physical state $f \in \mathrm{H}^{3, \mathrm{~N}}$ can be approximated with an arbitrary precision by the corresponding elements of the Cauchy sequence in $\mathrm{S}^{3, \mathrm{~N}}$, which converges to the given $f \in \mathrm{H}^{3, \mathrm{~N}}$. Further, taking into account the requirement to measure the arbitrary value of the quantum-mechanical model with non-absolute precision, it means that all concrete calculations can be fulfilled within the Schwartz test function space $\mathrm{S}^{3, \mathrm{~N}}$. Thus, such consideration allows us to perform, without any loss of generality, all necessary calculations in the space $\mathrm{S}^{3, \mathrm{~N}}$ at the level of correct differential and integral calculus.

Furthermore, the mathematical correctness of the consideration demands to determine the domain of definitions and the range of values for any used operator and for the functions of operators. Note that if the kernel space $\mathrm{S}^{3, \mathrm{~N}} \subset \mathrm{H}^{3, \mathrm{~N}}$ is taken as the common domain of definitions of the generating operators $\vec{x}=\left(x^{j}\right), \widehat{\vec{p}}=\left(\widehat{p}^{j}\right), \vec{s} \equiv$ $\left(s^{j}\right)=\left(s_{23}, s_{31}, s_{12}\right)$ of coordinate, momentum and spin, respectively, then this space appears to be also the range of their values. Moreover, the space $S^{3, N}$ appears to be the common domain of definitions and values for the set of all below mentioned functions from the 9 operators $\vec{x}=\left(x^{j}\right), \widehat{\vec{p}}=\left(\widehat{p}^{j}\right), \vec{s} \equiv\left(s^{j}\right)$ (for example, for the generators $\left(\widehat{p}_{\mu}, \widehat{j}_{\mu \nu}\right)$ of the irreducible unitary representations of the Poincaré group $\mathcal{P}$ and for different sets of commutation relations). Therefore, in order to guarantee the realization of the principle of correspondence between the results of cognition and the instruments of cognition in the given model, it is sufficient to take the algebra $A_{S}$ of the all sets of observables of the given model in the form of converged in $\mathrm{S}^{3, \mathrm{~N}}$ Hermitian power series of the 9 generating operators $\vec{x}=\left(x^{j}\right), \widehat{\vec{p}}=\left(\widehat{p}^{j}\right), \vec{s} \equiv\left(s^{j}\right)$.

Note that the Schrödinger-Foldy equation (3) has generalized solutions, which do not belong to the space $\mathrm{H}^{3, \mathrm{~N}}$ (10). Therefore, the application of the rigged Hilbert space $\mathrm{S}^{3, \mathrm{~N}} \subset \mathrm{H}^{3, \mathrm{~N}} \subset \mathrm{S}^{3, \mathrm{~N} *}(11)$ is necessary.

Some other details of motivations of the choice of the spaces (10), (11) (and all necessary notations) are given in [12], where the corresponding 4-component spaces are considered.

Note finally that in the case of arbitrary spin particleantiparticle doublet the dimension of spaces (10), (11) is $\mathrm{M}=2 \mathrm{~N}=2(2 \mathrm{~s}+1)$

\subsection{On the time evolution of the state vectors}

The time dependence of the state vectors $f \in \mathrm{H}^{3, \mathrm{~N}}$ (time $t$ is the parameter of evolution) is given either in the integral form by the unitary operator

$$
u\left(t_{0}, t\right)=\exp \left[-i \widehat{\omega}\left(t-t_{0}\right)\right] ; \quad \widehat{\omega} \equiv \sqrt{-\Delta+m^{2}},
$$

(below $t_{0}=t$ is put), or in the differential form by the Schrödinger-Foldy equation of motion (3) with the wave function (4). In terms of operator (5)-(7) this equation is given by

$$
\left(i \partial_{0}-\widehat{\omega}\right) f(x)=0 .
$$

Note that here the operator $\widehat{\omega} \equiv \sqrt{-\Delta+m^{2}}$ is the relativistic analog of the energy operator (Hamiltonian) of nonrelativistic quantum mechanics. The Minkowski space-time $\mathrm{M}(1,3)$ is pseudo Euclidean with metric $g=$ $\operatorname{diag}(+1,-1,-1,-1)$. The step from the particle singlet of arbitrary spin to the corresponding particle-antiparticle doublet is evident.

Thus, for the arbitrary spin particle-antiparticle doublet the system of two N-component equations $\left(i \partial_{0}-\widehat{\omega}\right) f(x)=$ 0 and $\left(i \partial_{0}-\widehat{\omega}\right) f(x)=0$ is used. Therefore, the corresponding Schrödinger-Foldy equation is given by (13), where the $2 \mathrm{~N}$-component wave function is the direct sum of the particle and antiparticle wave functions, respectively. Due to the historical tradition of the physicists the antiparticle wave function is put in the down part of the $2 \mathrm{~N}$-column.

The general solution of the Schrödinger-Foldy equation of motion (13) (in the case of particle-antiparticle arbitrary spin doublet) has the form

$$
\begin{array}{r}
f(x)=\frac{1}{(2 \pi)^{\frac{3}{2}}} \int d^{3} k e^{-i k x} a^{2 \mathrm{~N}}(\vec{k}) \mathrm{d}_{2 \mathrm{~N}}, \\
k x \equiv \omega t-\vec{k} \vec{x}, \quad \omega \equiv \sqrt{\vec{k}^{2}+m^{2}},
\end{array}
$$

where the orts of the $\mathrm{N}$-dimensional Cartesian basis are given in [1] by the formulas (10).

The action of the pseudo-differential (non-local) operator $\widehat{\omega} \equiv \sqrt{-\Delta+m^{2}}$ is explained in (6), (7).

\subsection{On the fundamental dynamical variables}

The dynamical variable $\vec{x} \in \mathrm{R}^{3} \subset \mathrm{M}(1,3)$ (as well as the variable $\vec{k} \in \mathrm{R}_{\vec{k}}^{3}$ ) represents the external degrees of freedom of the arbitrary spin particle-antiparticle doublet. The spin $\vec{s}$ of the particle-antiparticle doublet is the first in the list of the carriers of the internal degrees of freedom. Taking into account the Pauli principle and the fact that experimentally an antiparticle is observed as the mirror reflection of a particle, the operators of the charge sign and the spin of the arbitrary particle-antiparticle doublet are taken in the form

$$
\begin{gathered}
g \equiv-\Gamma_{2 \mathrm{~N}}^{0} \equiv-\sigma_{2 \mathrm{~N}}^{3}=\left|\begin{array}{ll}
-\mathrm{I}_{\mathrm{N}} & 0 \\
0 & \mathrm{I}_{\mathrm{N}}
\end{array}\right|, \\
\vec{s}_{2 \mathrm{~N}}=\left|\begin{array}{cc}
\vec{s}_{\mathrm{N}} & 0 \\
0 & -\hat{C} \vec{s}_{\mathrm{N}} \hat{C}
\end{array}\right|, \quad \mathrm{N}=2 s+1,
\end{gathered}
$$


where $\Gamma_{2 \mathrm{~N}}^{0}$ is the $2 \mathrm{~N} \times 2 \mathrm{~N}$ Dirac $\Gamma^{0}$ matrix, $\sigma_{2 \mathrm{~N}}^{3}$ is the $2 \mathrm{~N} \times 2 \mathrm{~N}$ Pauli $\sigma^{3}$ matrix, $\hat{C}$ is the operator of complex conjugation in the form of $\mathrm{N} \times \mathrm{N}$ diagonal matrix, the operator of involution in $\mathrm{H}^{3,2 \mathrm{~N}}$, and $\mathrm{I}_{\mathrm{N}}$ is $\mathrm{N} \times \mathrm{N}$ unit matrix.

Thus, the spin is given by the generators of $\mathrm{SU}(2)$ algebra!

The spin matrices $\vec{s}_{2 \mathrm{~N}}(15)$ satisfy the commutation relations

$$
\left[s_{2 \mathrm{~N}}^{j}, s_{2 \mathrm{~N}}^{\ell}\right]=i \varepsilon^{j \ell n} s_{2 \mathrm{~N}}^{n}, \quad \varepsilon^{123}=+1,
$$

of the algebra of $\mathrm{SU}(2)$ group, where $\varepsilon^{j \ell n}$ is the Levi-Civita tensor and $s^{j}=\varepsilon^{j \ell n} s_{\ell n}$ are the Hermitian $2 \mathrm{~N} \times 2 \mathrm{~N}$ matrices $(15)$ - the generators of a $2 \mathrm{~N}$-dimensional reducible representation of the spin group $\mathrm{SU}(2)$ (universal covering of the $\mathrm{SO}(3) \subset \mathrm{SO}(1,3)$ group).

The Casimir operator for the RCQM representation of $\mathrm{SU}(2)$ spin given in (15) has the form

$$
\vec{s}_{2 \mathrm{~N}}^{2}=\mathrm{s}(\mathrm{s}+1) \mathrm{I}_{2 \mathrm{~N}},
$$

where $\mathrm{I}_{2 \mathrm{~N}}$ is $2 \mathrm{~N} \times 2 \mathrm{~N}$ unit matrix.

Above in the text of this axiom the case of arbitrary spin particle-antiparticle doublet has been considered. For the case of arbitrary spin particle singlet the operator of the charge sign is absent. The spin operator $\vec{s}_{\mathrm{N}}$ is given by the Hermitian $\mathrm{N} \times \mathrm{N}$ matrices - the generators of a $\mathrm{N}$ dimensional irreducible representation of the spin group $\mathrm{SU}(2)$. Therefore, the $\mathrm{SU}(2)$ commutation relations in such notations have the explicit form

$$
\left[s_{\mathrm{N}}^{j}, s_{\mathrm{N}}^{\ell}\right]=i \varepsilon^{j \ell n} s_{\mathrm{N}}^{n}, \quad \varepsilon^{123}=+1,
$$

and the corresponding Casimir operator is given by

$$
\vec{s}_{\mathrm{N}}^{2}=\mathrm{s}(\mathrm{s}+1) \mathrm{I}_{\mathrm{N}} \text {. }
$$

The validity of these assertions is proved by numerous partial examples presented in [1].

\subsection{On the external and internal degrees of freedom}

The coordinate $\vec{x}$ (as an operator in $\mathrm{H}^{3,2 \mathrm{~N}}$ ) is an ana$\log$ of the discrete index of generalized coordinates $q \equiv$ $\left(q_{1}, q_{2}, \ldots\right)$ in non-relativistic quantum mechanics of the finite number degrees of freedom. In other words the coordinate $\vec{x} \in \mathrm{R}^{3} \subset \mathrm{M}(1,3)$ is the continuous carrier of the external degrees of freedom of a multiplet (the similar consideration was given in [81]). The coordinate operator together with the operator $\widehat{\vec{p}}$ determines the operator $m_{l n}=x_{l} \widehat{p}_{n}-x_{n} \widehat{p}_{l}$ of an orbital angular momentum, which also is connected with the external degrees of freedom.

However, the RCQM doublet has the additional characteristics such as the spin operator $\vec{s}(15)$, which is the carrier of the internal degrees of freedom of this multiplet. The set of generators $\left(\widehat{p}_{\mu}, \widehat{j}_{\mu \nu}\right)$ (formulas (22), (23) below) of the main dynamical variables (formulas (73) in [1]) of the doublet are the functions of the following basic set of 9 functionally independent operators

$$
\vec{x}=\left(x^{j}\right), \widehat{\vec{p}}=\left(\widehat{p}^{j}\right), \vec{s}_{2 \mathrm{~N}} \equiv\left(s_{2 \mathrm{~N}}^{j}\right)=\left(s_{23}, s_{31}, s_{12}\right) .
$$

Note that spin $\vec{s}_{2 \mathrm{~N}}(15)$ commutes both with $(\vec{x}, \widehat{\vec{p}})$ and with the operator $i \partial_{t}-\sqrt{-\Delta+m^{2}}$ of the SchrödingerFoldy equation (13). Thus, for the free doublet the external and internal degrees of freedom are independent. Therefore, 9 operators (20) in $\mathrm{H}^{3,2 \mathrm{~N}}$, which have the univocal physical sense, are the generating operators not only for the $10 \mathcal{P}$ generators $\left(\widehat{p}_{\mu}, \widehat{j}_{\mu \nu}\right)(22),(23)$, but also for other operators of any experimentally observable quantities of the doublet.

\subsection{On the algebra of observables}

Using the operators of canonically conjugated coordinate $\vec{x}$ and momentum $\vec{p}$ (where $\left[x^{j}, \widehat{p}^{\ell}\right]=i \delta^{j \ell}, \quad\left[x^{j}, x^{\ell}\right]=$ $\left[\widehat{p}^{j}, \widehat{p}^{\ell}\right]=0$, ) in $\mathrm{H}^{3,2 \mathrm{~N}}$, being completed by the operators $\vec{s}_{2 \mathrm{~N}}$ and $g(15)$, we construct the algebra of observables as the Hermitian functions of $10\left(\vec{x}, \vec{p}, \vec{s}_{2 \mathrm{~N}},-\Gamma_{2 \mathrm{~N}}^{0}\right)$ generating elements of the algebra.

\subsection{On the relativistic invariance of the the- ory}

The relativistic invariance of the model under consideration (the relativistic invariance of the SchrödingerFoldy equation (13)) requires, as a first step, consideration of its invariance with respect to the proper ortochronous Lorentz $\mathrm{L}_{+}^{\uparrow}=\operatorname{SO}(1,3)=\left\{\Lambda=\left(\Lambda_{\nu}^{\mu}\right)\right\}$ and Poincaré $\mathrm{P}_{+}^{\uparrow}=$ $\mathrm{T}(4) \times) \mathrm{L}_{+}^{\uparrow} \supset \mathrm{L}_{+}^{\uparrow}$ groups. This invariance in an arbitrary relativistic model is the implementation of the Einstein's relativity principle in the special relativity form. Note that the mathematical correctness requires the invariance mentioned above to be considered as the invariance with respect to the universal coverings $\mathcal{L}=\operatorname{SL}(2, \mathrm{C})$ and $\mathcal{P} \supset \mathcal{L}$ of the groups $\mathrm{L}_{+}^{\uparrow}$ and $\mathrm{P}_{+}^{\uparrow}$, respectively.

For the group $\mathcal{P}$ we choose real parameters $a=$ $\left(a^{\mu}\right) \in \mathrm{M}(1,3)$ and $\varpi \equiv\left(\varpi^{\mu \nu}=-\varpi^{\nu \mu}\right)$ with well-known physical meaning. For the standard $\mathcal{P}$ generators $\left(p_{\mu}, j_{\mu \nu}\right)$ we use commutation relations in the manifestly covariant form

$$
\begin{gathered}
{\left[p_{\mu}, p_{\nu}\right]=0,\left[p_{\mu}, j_{\rho \sigma}\right]=i g_{\mu \rho} p_{\sigma}-i g_{\mu \sigma} p_{\rho},} \\
{\left[j_{\mu \nu}, j_{\rho \sigma}\right]=-i\left(g_{\mu \rho} j_{\nu \sigma}+g_{\rho \nu} j_{\sigma \mu}+g_{\nu \sigma} j_{\mu \rho}+g_{\sigma \mu} j_{\rho \nu}\right) .}
\end{gathered}
$$

The following assertion should be noted. Not a matter of fact that non-covariant objects such as the Lebesgue measure $d^{3} x$ and non-covariant (non-Lie) generators of algebras are explored, the model of RCQM of arbitrary spin is a relativistic invariant in the following sense. The Schrödinger-Foldy equation (13) and the set of its solution $\{f\}(14)$ are invariant with respect to the irreducible 
unitary representation of the group $\mathcal{P}$, the $\mathrm{N} \times \mathrm{N}$ matrixdifferential generators of which are given by the following nonlocal operators

$$
\begin{gathered}
\widehat{p}_{0}=\widehat{\omega} \equiv \sqrt{-\Delta+m^{2}}, \quad \widehat{p}_{\ell}=i \partial_{\ell}, \\
\widehat{j}_{\ell n}=x_{\ell} \widehat{p}_{n}-x_{n} \widehat{p}_{\ell}+s_{l n} \equiv \widehat{m}_{\ell n}+s_{\ell n}, \\
\widehat{j}_{0 \ell}=-\widehat{j}_{\ell 0}=t \widehat{p}_{\ell}-\frac{1}{2}\left\{x_{\ell}, \widehat{\omega}\right\}-\left(\frac{s_{\ell n} \widehat{p}_{n}}{\widehat{\omega}+m} \equiv \breve{s}_{\ell}\right),
\end{gathered}
$$

where the orbital parts of the generators are not changed under the transition from one spin to another. Under such transitions only the spin parts (15), (16) of the expressions (22), (23) are changed. Indeed, the direct calculations visualize that generators (22), (23) commute with the operator of equation (13) and satisfy the commutation relations (21) of the Lie algebra of the Poincare group $\mathcal{P}$. In formulas (22), (23), the SU(2)-spin generators $s^{\ell n}$ have particular specific forms for each representation of the $\mathrm{SU}(2)$ group (see the list of examples in [1]).

Note that the generators (22), (23) are known from the formulae (B-25) (B-28) of the paper [5].

Thus, the irreducible unitary representation of the Poincare group $\mathcal{P}$ in the space (11), with respect to which the Schrödinger-Foldy equation (13) and the set of its solution $\{f\}$ (14) are invariant, is given by a series converges in this space

$$
(a, \varpi) \rightarrow U(a, \varpi)=\exp \left(-i a^{0} \widehat{p}_{0}-i \vec{a} \widehat{\vec{p}}-\frac{i}{2} \varpi^{\mu \nu} \widehat{j}_{\mu \nu}\right)
$$

where the generators $\left(\widehat{p}^{\mu}, \widehat{j}^{\mu \nu}\right)$ are given in (22), (23) with the arbitrary values of the SU(2) spins $\vec{s}=\left(s^{\ell n}\right)(15)$, (16).

The validity of this assertion is verified by the following three steps. (i) The calculation that the $\mathcal{P}$-generators (22), (23) commute with the operator $i \partial_{0}-\widehat{\omega}$ of the SchrödingerFoldy equation (13). (ii) The verification that the $\mathcal{P}$ generators (22), (23) satisfy the commutation relations (21) of the Lie algebra of the Poincaré group $\mathcal{P}$. (iii) The proof that generators (22), (23) realize the spin $s(s+1)$ representation of this group. Therefore, the Bargman-Wigner classification on the basis of the corresponding Casimir operators calculation should be given. These three steps can be made by direct and non-cumbersome calculations.

The expression (24) is well known, but rather formal. In fact the transition from a Lie algebra to a finite group transformations in the case of non-Lie generators is a rather non-trivial action. The mathematical justification of (24) can be fulfilled in the framework of Schwartz test function space and will be given in next special publication.

The corresponding Casimir operators have the form

$$
\begin{gathered}
p^{2}=\widehat{p}^{\mu} \widehat{p}_{\mu}=m^{2} \mathrm{I}_{\mathrm{N}}, \\
W=w^{\mu} w_{\mu}=m^{2} \vec{s}^{2}=\mathrm{s}(\mathrm{s}+1) m^{2} \mathrm{I}_{\mathrm{N}},
\end{gathered}
$$

where $\mathrm{I}_{\mathrm{N}}$ is the $\mathrm{N} \times \mathrm{N}$ unit matrix and $\mathrm{s}=1 / 2,1,3 / 2,2, \ldots$
Note that together with the generators (22), (23) another set of 10 operators commutes with the operator of equation (13), satisfies the commutation relations (21) of the Lie algebra of Poincaré group $\mathcal{P}$, and, therefore, can be chosen as the Poincaré symmetry of the model under consideration. This second set is given by the generators $\widehat{p}^{0}, \widehat{p}^{\ell}$ from (22) together with the orbital parts of the generators $\hat{j}^{\ell n}, \hat{j}^{0 \ell}$ from (23). Thus, this second set of Poincaré generators is given by

$$
\begin{gathered}
\widehat{p}_{0}=\widehat{\omega} \equiv \sqrt{-\Delta+m^{2}}, \quad \widehat{p}_{\ell}=i \partial_{\ell}, \\
\widehat{m}_{\ell n}=x_{\ell} \widehat{p}_{n}-x_{n} \widehat{p}_{\ell}, \quad \widehat{m}_{0 \ell}=-\widehat{m}_{\ell 0}=t \widehat{p}_{\ell}-\frac{1}{2}\left\{x_{\ell}, \widehat{\omega}\right\} .
\end{gathered}
$$

Note that in the case $s=0$ only generators (27) form the Poincaré symmetry.

Note that the modern definition of $\mathcal{P}$ invariance (or $\mathcal{P}$ symmetry) of the equation of motion (13) in $\mathrm{H}^{3, \mathrm{~N}}$ is given by the following assertion, see, e. g. [82]. The set $\mathrm{F} \equiv\{f\}$ of all possible solutions of the equation (13) is invariant with respect to the $\mathcal{P}^{\mathrm{f}}$-representation of the group $\mathcal{P}$, if for arbitrary solution $f$ and arbitrarily-fixed parameters $(a, \varpi)$ the assertion

$$
(a, \varpi) \rightarrow U(a, \varpi)\{f\}=\{f\} \equiv \mathrm{F}
$$

is valid.

\subsection{On the dynamic and kinematic aspects of the relativistic invariance}

Consider briefly some detalizations of the relativistic invariance of the Schrödinger-Foldy equation (13). Note that for the free particle-antiparticle doublet of arbitrary spin the equation (13) has one and the same explicit form in arbitrary-fixed IFR (its set of solutions is one and the same in every IFR). Therefore, the algebra of observables and the conservation laws (as the functionals of the free particleantiparticle doublet states) have one and the same form too. This assertion explains the dynamical sense of the $\mathcal{P}$ invariance (the invariance with respect to the dynamical symmetry group $\mathcal{P}$ ).

Another, kinematic, aspect of the $\mathcal{P}$ invariance of the RQCM model has the following physical sense. Note at first that any solution of the Schrödinger-Foldy equation (13) is determined by the concrete given set of the amplitudes $\{A\}$. It means that if $f$ with the fixed set of amplitudes $\{A\}$ is the state of the doublet in some arbitrary IFR, then for the observer in the $(a, \varpi)$-transformed IFR' this state $f^{\prime}$ is determined by the amplitudes $\left\{A^{\prime}\right\}$. The last ones are received from the given $\{A\}$ by the unitary $\mathcal{P}^{\mathrm{A}}$ -transformation (24).

\subsection{On the Clifford-Dirac algebra}

This axiom is additional and is not necessary. Nevertheless, such axiom is very useful for the dimensions, where the $\Gamma$ matrices exist. 
Application of the Clifford-Dirac algebra is the useful method of calculations in RCQM. Three different definitions of the Clifford algebra and their equivalence are considered in [83]. In different approaches to the relativistic quantum mechanics the matrix representation of the Clifford algebra in terms of the Dirac gamma matrices is used. This representation is called the Clifford-Dirac algebra.

For our purposes the anti-commutation relations of the Clifford-Dirac algebra generators are taken in the general form

$$
\begin{gathered}
\Gamma_{2 \mathrm{~N}}^{\bar{\mu}} \Gamma_{2 \mathrm{~N}}^{\bar{\nu}}+\Gamma_{2 \mathrm{~N}}^{\bar{\nu}} \Gamma_{2 \mathrm{~N}}^{\bar{\mu}}=2 g^{\bar{\mu} \bar{\nu}} ; \\
\bar{\mu}, \bar{\nu}=\overline{0,4}, \quad\left(g^{\bar{\mu} \bar{\nu}}\right)=(+----),
\end{gathered}
$$

where $\Gamma_{2 \mathrm{~N}}^{\bar{\mu}}$ are the $2 \mathrm{~N} \times 2 \mathrm{~N}$ Dirac $\Gamma^{\bar{\mu}}$ matrices $(2 \mathrm{~N} \times 2 \mathrm{~N}$ generalization of the Dirac $4 \times 4 \gamma$ matrices), $\Gamma_{2 \mathrm{~N}}^{4} \equiv$ $\Gamma_{2 \mathrm{~N}}^{0} \Gamma_{2 \mathrm{~N}}^{1} \Gamma_{2 \mathrm{~N}}^{2} \Gamma_{2 \mathrm{~N}}^{3}$. Here and in our publications (see, e. g. the articles [84-91]) we use the $\gamma^{4} \equiv \gamma^{0} \gamma^{1} \gamma^{2} \gamma^{3}$ matrix instead of the $\gamma^{5}$ matrix of other authors. Our $\gamma^{4}$ is equal to $i \gamma_{\text {standard }}^{5}$. Notation $\gamma^{5}$ is used in [84-91] for a completely different matrix $\gamma^{5} \equiv \gamma^{1} \gamma^{3} \hat{C}$. As well as the element $\Gamma_{2 \mathrm{~N}}^{4} \equiv \Gamma_{2 \mathrm{~N}}^{0} \Gamma_{2 \mathrm{~N}}^{1} \Gamma_{2 \mathrm{~N}}^{2} \Gamma_{2 \mathrm{~N}}^{3}$ of (29) is dependent the algebra basis is formed by $4=1+3$ independent elements. Therefore, such Clifford algebra over the field of complex numbers is denoted $\mathrm{Cl}^{\mathrm{C}}(1,3)$ and the dimension of the algebra is $2^{4}=16$.

Note that relations (29) are valid only for the dimensions, where the $\Gamma_{2 \mathrm{~N}}$ are defined (where the matrix representation of the Clifford algebra exists). Therefore, the relations (29) can be useful not for all multiplets of RCQM. Nevertheless, existing relations (29) are very useful in order to operate with spins, with standard FW transformation and in order to formulate the $\mathrm{FW}$ transformation generalizations for the dimensions $2(2 s+1)>4$.

It has been explained in [1] (and in [85-91] in details) that the Clifford-Dirac algebra should be introduced into consideration in the FW representation [6] of the spinor field. The reasons are as follows. Part of the Clifford-Dirac algebra operators are directly related to the spin $1 / 2$ doublet operators $\left(s^{1} \equiv \frac{1}{2} \gamma^{2} \gamma^{3}, s^{2} \equiv \frac{1}{2} \gamma^{3} \gamma^{1}, s^{3} \equiv \frac{1}{2} \gamma^{1} \gamma^{2}\right)$ (in the anti-Hermitian form). In the FW representation for the spinor field [6] these spin operators commute with the Hamiltonian and with the operator of the FW equation of motion $i \partial_{0}-\gamma^{0} \widehat{\omega}$. In the Pauli-Dirac representation these operators do not commute with the Dirac equation operator. Only the sums of the orbital operators and such spin operators commute with the Diracian. So if we want to relate the orts $\gamma^{\mu}$ of the Clifford-Dirac algebra with the actual spin we must introduce this algebra into the FW representation.

Here in general N-dimensional formalism of RCQM the situation is similar. The anti-commutation relations of the Clifford-Dirac algebra generators (29) and corresponding $\Gamma_{2 \mathrm{~N}}$ matrices must be introduced in the FW representation. Therefore, in order to apply (29) in RCQM one must transform the $\Gamma_{2 \mathrm{~N}}$ matrices from the $\mathrm{FW}$ representation into the RCQM representation. Corresponding operator transfor- mation is given by

$$
\begin{aligned}
v_{2 \mathrm{~N}}= & \left|\begin{array}{ll}
\mathrm{I}_{\mathrm{N}} & 0 \\
0 & \hat{C} \mathrm{I}_{\mathrm{N}}
\end{array}\right|, \quad v_{2 \mathrm{~N}}^{-1}=v_{2 \mathrm{~N}}^{\dagger}=v_{2 \mathrm{~N}}, \\
& v_{2 \mathrm{~N}} v_{2 \mathrm{~N}}=\mathrm{I}_{2 \mathrm{~N}}, \quad \mathrm{~N}=2 s+1,
\end{aligned}
$$

where $\hat{C} \mathrm{I}_{\mathrm{N}}$ is the $\mathrm{N} \times \mathrm{N}$ operator of complex conjugation. Indeed, the operator (30) translates any operator from canonical field FW representation into the RCQM representation and vice versa:

$$
\begin{aligned}
& v_{2 \mathrm{~N}} \hat{q}_{\mathrm{cf}}^{\text {anti-Herm }} v_{2 \mathrm{~N}}=\hat{q}_{\mathrm{qm}}^{\text {anti-Herm }}, \\
& v_{2 \mathrm{~N}} \hat{q}_{\mathrm{qm}}^{\text {anti-Herm }} v_{2 \mathrm{~N}}=\hat{q}_{\mathrm{cf}}^{\text {anti-Herm }} .
\end{aligned}
$$

Here $\hat{q}_{\mathrm{qm}}^{\text {anti-Herm }}$ is an arbitrary operator from the RCQM of the $2 \mathrm{~N}$-component particle-antiparticle doublet in the antiHermitian form, e. g., the operator $\left(\partial_{0}+i \widehat{\omega}\right)$ of equation of motion (13), the operator of spin $\vec{s}_{2 \mathrm{~N}}$ (15) taken in anti-Hermitian form, etc., $\hat{q}_{\mathrm{cf}}^{\text {anti-Herm }}$ is an arbitrary operator from the canonical field theory of the $2 \mathrm{~N}$-component particle-antiparticle doublet in the anti-Hermitian form. Thus, the only warning is that operators here must be taken in anti-Hermitian form, see section 9 in [1] for the details and see $[92,93]$ for the mathematical correctness of antiHermitian operators application (note that contrary to [94, 95], where non-Hermitian operators have been considered, we appeal in $[1-3,11,12]$ and here only to anti-Hermitian operators and corresponding Foldy-Wouthuysen transformation).

Further, the operator (30) translates

$$
\phi=v_{2 \mathrm{~N}} f, \quad f=v_{2 \mathrm{~N}} \phi,
$$

the solution (14) of the Schrödinger-Foldy equation (13) into the solution

$\phi(x)=\frac{1}{(2 \pi)^{\frac{3}{2}}} \int d^{3} k\left[e^{-i k x} a^{\mathrm{N}}(\vec{k}) \mathrm{d}_{\mathrm{N}}+e^{i k x} a^{* \breve{\mathrm{N}}}(\vec{k}) \mathrm{d}_{\breve{\mathrm{N}}}\right]$,

$\mathrm{N}=1,2, \ldots, \mathrm{N}, \quad \breve{\mathrm{N}}=\mathrm{N}+1, \mathrm{~N}+2, \ldots, 2 \mathrm{~N}$, of the $\mathrm{FW}$ equation

$$
\left(i \partial_{0}-\Gamma_{2 \mathrm{~N}}^{0} \widehat{\omega}\right) \phi(x)=0, \quad \Gamma_{2 \mathrm{~N}}^{0} \equiv \sigma_{2 \mathrm{~N}}^{3}=\left|\begin{array}{ll}
\mathrm{I}_{\mathrm{N}} & 0 \\
0 & -\mathrm{I}_{\mathrm{N}}
\end{array}\right|,
$$

$\widehat{\omega} \equiv \sqrt{-\Delta+m^{2}}, \quad \mathrm{~N}=2 s+1$, and vice versa. Thus, the transformation (30), (31) translates the matrices $\Gamma_{2 \mathrm{~N}}^{0}$ and

$$
\Gamma_{2 \mathrm{~N}}^{j}=\left|\begin{array}{lc}
0 & \Sigma_{\mathrm{N}}^{j} \\
-\Sigma_{\mathrm{N}}^{j} & 0
\end{array}\right|, \quad j=1,2,3,
$$

into the RCQM representation

$$
\bar{\Gamma}_{2 \mathrm{~N}}^{\bar{\mu}}=v_{2 \mathrm{~N}} \Gamma_{2 \mathrm{~N}}^{\bar{\mu}} v_{2 \mathrm{~N}},
$$

where matrices $\bar{\Gamma}_{2 \mathrm{~N}}^{\bar{\mu}}$ satisfy the anti-commutation relations

$$
\bar{\Gamma}_{2 \mathrm{~N}}^{\bar{\mu}} \bar{\Gamma}_{2 \mathrm{~N}}^{\bar{\nu}}+\bar{\Gamma}_{2 \mathrm{~N}}^{\bar{\nu}} \bar{\Gamma}_{2 \mathrm{~N}}^{\bar{\mu}}=2 g^{\bar{\mu} \bar{\nu}} ;
$$




$$
\bar{\mu}, \bar{\nu}=\overline{0,4}, \quad\left(g^{\bar{\mu} \bar{\nu}}\right)=(+----),
$$

of the Clifford-Dirac algebra generators as well. In (35) $\Sigma_{\mathrm{N}}^{j}$ are the $\mathrm{N} \times \mathrm{N}$ Pauli matrices. Note that Pauli matrices exist only if the values of $\mathrm{N}$ are even. The explicit forms of the RCQM representation of the $\bar{\Gamma}_{2 \mathrm{~N}}^{\bar{\mu}}$ matrices are given by

$$
\begin{gathered}
\bar{\Gamma}_{2 \mathrm{~N}}^{0}=\Gamma_{2 \mathrm{~N}}^{0}, \quad \bar{\Gamma}_{2 \mathrm{~N}}^{1}=\Gamma_{2 \mathrm{~N}}^{1} \widehat{C}, \quad \bar{\Gamma}_{2 \mathrm{~N}}^{2}=\Gamma_{2 \mathrm{~N}}^{0} \Gamma_{2 \mathrm{~N}}^{2} \widehat{C}, \\
\bar{\Gamma}_{2 \mathrm{~N}}^{3}=\Gamma_{2 \mathrm{~N}}^{3} \widehat{C}, \quad \bar{\Gamma}_{2 \mathrm{~N}}^{4}=\Gamma_{2 \mathrm{~N}}^{0} \Gamma_{2 \mathrm{~N}}^{4} \widehat{C},
\end{gathered}
$$

where $\widehat{C}$ is the $2 \mathrm{~N} \times 2 \mathrm{~N}$ operator of complex conjugation and matrices $\Gamma_{2 \mathrm{~N}}^{\bar{\mu}}$ are given in (34), (35).

Note that in the terms of $\bar{\Gamma}_{2 \mathrm{~N}}^{\bar{\mu}}$ matrices (38) the RCQM spin operator (15) has the form

$$
\vec{s}=\frac{i}{2}\left(\bar{\Gamma}_{2 \mathrm{~N}}^{2} \bar{\Gamma}_{2 \mathrm{~N}}^{3}, \bar{\Gamma}_{2 \mathrm{~N}}^{3} \bar{\Gamma}_{2 \mathrm{~N}}^{1}, \bar{\Gamma}_{2 \mathrm{~N}}^{1} \bar{\Gamma}_{2 \mathrm{~N}}^{2}\right) .
$$

Note further that formula (39) is valid for the multiplets of arbitrary dimension but only for the spin $s=1 / 2$, whereas the formula (15) is valid for arbitrary spin. Note also that in some dimensions the gamma matrices can not be defined. Furthermore, the complete analogy between the (39) and the particle-antiparticle spin $s=1 / 2$ doublet of arbitrary dimension in the FW representation exists

$$
\vec{s}_{\mathrm{FW}}=\frac{i}{2}\left(\Gamma_{2 \mathrm{~N}}^{2} \Gamma_{2 \mathrm{~N}}^{3}, \Gamma_{2 \mathrm{~N}}^{3} \Gamma_{2 \mathrm{~N}}^{1}, \Gamma_{2 \mathrm{~N}}^{1} \Gamma_{2 \mathrm{~N}}^{2}\right) .
$$

It is very useful to consider a wider then $\mathrm{Cl}^{\mathrm{C}}(1,3)$ CliffordDirac algebra. In [85-91] such additional algebras have been introduced for the purposes of finding links between the fermionic and bosonic states of the spinor field. New algebra can be formed by the generators of $\mathrm{Cl}^{\mathrm{C}}(1,3)$ together with the generators of the Pauli-Gürsey-Ibragimov algebra [96-98].

The main structure elements of such set are given by $\left(\gamma^{0}, \gamma^{1}, \gamma^{2}, \gamma^{3}, i, \hat{C} \mathrm{I}_{4}\right)$, where $\gamma^{\mu}$ are $4 \times 4$ Dirac matrices in standard representation. It is easy to see that simplest set of the Clifford-Dirac algebra generators can be constructed from these elements in the form $\left(i \gamma^{0}, i \gamma^{1}, \gamma^{2}, i \gamma^{3}, \hat{C} \mathrm{I}_{4}, i \hat{C} \mathrm{I}_{4}\right)$. Therefore, the $2 \mathrm{~N} \times 2 \mathrm{~N}$ matrix generators of the corresponding Clifford-Dirac algebra over the field of real numbers can be found by the simple redefinition

$$
\begin{gathered}
\tilde{\Gamma}_{2 \mathrm{~N}}^{1} \equiv i \Gamma_{2 \mathrm{~N}}^{1}, \quad \tilde{\Gamma}_{2 \mathrm{~N}}^{2} \equiv i \Gamma_{2 \mathrm{~N}}^{3}, \quad \tilde{\Gamma}_{2 \mathrm{~N}}^{3} \equiv \widehat{C} \mathrm{I}_{2 \mathrm{~N}}, \\
\tilde{\Gamma}_{2 \mathrm{~N}}^{4} \equiv i \widehat{C} \mathrm{I}_{2 \mathrm{~N}}, \quad \tilde{\Gamma}_{2 \mathrm{~N}}^{5} \equiv i \Gamma_{2 \mathrm{~N}}^{0}, \quad \tilde{\Gamma}_{2 \mathrm{~N}}^{6} \equiv-\Gamma_{2 \mathrm{~N}}^{2},
\end{gathered}
$$

of the matrices $\left(i \Gamma_{2 \mathrm{~N}}^{0}, i \Gamma_{2 \mathrm{~N}}^{1}, \Gamma_{2 \mathrm{~N}}^{2}, i \Gamma_{2 \mathrm{~N}}^{3}, \widehat{C} \mathrm{I}_{2 \mathrm{~N}}, i \widehat{C} \mathrm{I}_{2 \mathrm{~N}}\right)$, where $\Gamma_{2 \mathrm{~N}}^{\mu}$ are given in (34), (35).

Matrices (41) together with the matrix $\tilde{\Gamma}_{2 \mathrm{~N}}^{7} \equiv$ $\tilde{\Gamma}_{2 \mathrm{~N}}^{1} \tilde{\Gamma}_{2 \mathrm{~N}}^{2} \tilde{\Gamma}_{2 \mathrm{~N}}^{3} \tilde{\Gamma}_{2 \mathrm{~N}}^{4} \tilde{\Gamma}_{2 \mathrm{~N}}^{5} \tilde{\Gamma}_{2 \mathrm{~N}}^{6}=\Gamma_{2 \mathrm{~N}}^{4}$ satisfy the anticommutation relations of the Clifford-Dirac algebra generators in the form

$$
\begin{gathered}
\tilde{\Gamma}_{2 \mathrm{~N}}^{\mathrm{A}} \tilde{\Gamma}_{2 \mathrm{~N}}^{\mathrm{B}}+\tilde{\Gamma}_{2 \mathrm{~N}}^{\mathrm{B}} \tilde{\Gamma}_{2 \mathrm{~N}}^{\mathrm{A}}=2 g^{\mathrm{AB}} ; \\
\mathrm{A}, \mathrm{B}=\overline{1,7}, \quad\left(g^{\mathrm{AB}}\right)=(++++---) .
\end{gathered}
$$

As well as in (29) among the generators of (42) only the $4+2=6$ matrices (41) are independent and form the basis of the algebra. Therefore, the found above algebra over the field of real numbers is defined as $\mathrm{Cl}^{\mathrm{R}}(4,2)$ and the dimension of this algebra is $2^{6}=64$.

Useful realization of (41), (42) is given in terms of completely anti-Hermitian generators

$$
\begin{gathered}
\Gamma_{2 \mathrm{~N}}^{1}, \Gamma_{2 \mathrm{~N}}^{2}, \Gamma_{2 \mathrm{~N}}^{3}, \Gamma_{2 \mathrm{~N}}^{4}=\Gamma_{2 \mathrm{~N}}^{0} \Gamma_{2 \mathrm{~N}}^{1} \Gamma_{2 \mathrm{~N}}^{2} \Gamma_{2 \mathrm{~N}}^{3}, \\
\Gamma_{2 \mathrm{~N}}^{5}=\Gamma_{2 \mathrm{~N}}^{1} \Gamma_{2 \mathrm{~N}}^{3} \widehat{C}, \Gamma_{2 \mathrm{~N}}^{6}=i \Gamma_{2 \mathrm{~N}}^{1} \Gamma_{2 \mathrm{~N}}^{3} \widehat{C}, \Gamma_{2 \mathrm{~N}}^{7}=i \Gamma_{2 \mathrm{~N}}^{0},
\end{gathered}
$$

where matrices $\left\{\Gamma_{2 \mathrm{~N}}^{\mu}\right\}$ are given in (34), (35). Matrices (43) obey the anti-commutation relations of 64-dimensional $\mathrm{Cl}^{\mathrm{R}}(0,6)$ algebra in the form

$$
\Gamma_{2 \mathrm{~N}}^{\mathrm{A}} \Gamma_{2 \mathrm{~N}}^{\mathrm{B}}+\Gamma_{2 \mathrm{~N}}^{\mathrm{B}} \Gamma_{2 \mathrm{~N}}^{\mathrm{A}}=-2 \delta^{\mathrm{AB}}, \quad \mathrm{A}, \mathrm{B}=\overline{1,7} .
$$

Note that here as well only 6 operators are independent generators: $\Gamma_{2 \mathrm{~N}}^{4}=-i \Gamma_{2 \mathrm{~N}}^{7} \Gamma_{2 \mathrm{~N}}^{1} \Gamma_{2 \mathrm{~N}}^{2} \Gamma_{2 \mathrm{~N}}^{3}$.

Operators (43) generate also the 28 orts $s^{\overline{\mathrm{A}} \overline{\mathrm{B}}}$ :

$$
s^{\overline{\mathrm{A}} \overline{\mathrm{B}}}=\left\{s^{\mathrm{AB}}=\frac{1}{4}\left[\Gamma_{2 \mathrm{~N}}^{\mathrm{A}}, \Gamma_{2 \mathrm{~N}}^{\mathrm{B}}\right], s^{\mathrm{A} 8}=-s^{8 \mathrm{~A}}=\frac{1}{2} \Gamma_{2 \mathrm{~N}}^{\mathrm{A}}\right\},
$$

where $\overline{\mathrm{A}}, \overline{\mathrm{B}}=\overline{1,8}$ and generators $s^{\overline{\mathrm{A}} \overline{\mathrm{B}}}$ satisfy the commutation relations of $\mathrm{SO}(8)$ algebra

$$
\left[s^{\overline{\mathrm{A}} \overline{\mathrm{B}}}, s^{\overline{\mathrm{C}} \overline{\mathrm{D}}}\right]=\delta^{\overline{\mathrm{A}} \overline{\mathrm{C}}} s^{\overline{\mathrm{B}} \overline{\mathrm{D}}}+\delta^{\overline{\mathrm{C}} \overline{\mathrm{B}}} s^{\overline{\mathrm{D}} \overline{\mathrm{A}}}+\delta^{\overline{\mathrm{B}} \overline{\mathrm{D}}} s^{\overline{\mathrm{A}} \overline{\mathrm{C}}}+\delta^{\overline{\mathrm{D}} \overline{\mathrm{A}}} s^{\overline{\mathrm{C}} \overline{\mathrm{B}}} .
$$

Of course, the matrix representation (45) of the $\mathrm{SO}(8)$ algebra is considered over the field of real numbers.

Note that $\mathrm{SO}(8)$ algebra is not any subalgebra of the Clifford algebra $\mathrm{Cl}^{\mathrm{R}}(0,6)$. It is impossible in principle. Indeed, the mathematical object $\mathrm{Cl}^{\mathrm{R}}(0,6)$ belongs to the Clifford algebras and mathematical object $\mathrm{SO}(8)$ belongs to the Lie algebras.

As the consequences of the equalities

$$
\Gamma_{2 \mathrm{~N}}^{4} \equiv \prod_{\mu=0}^{3} \Gamma_{2 \mathrm{~N}}^{\mu} \rightarrow \prod_{\bar{\mu}=0}^{4} \Gamma_{2 \mathrm{~N}}^{\bar{\mu}}=-\mathrm{I},
$$

known from the standard Clifford-Dirac algebra $\mathrm{Cl}^{\mathrm{C}}(1,3)$, and the anticommutation relations $(44)$, in $\mathrm{Cl}^{\mathrm{R}}(0,6)$ algebra for the matrices $\Gamma_{2 \mathrm{~N}}^{\mathrm{A}}(43)$ the following extended equalities are valid:

$$
\Gamma_{2 \mathrm{~N}}^{7} \equiv-\prod_{\underline{\mathrm{A}}=1}^{6} \Gamma_{2 \mathrm{~N}}^{\mathrm{A}} \rightarrow \prod_{\mathrm{A}=1}^{7} \Gamma_{2 \mathrm{~N}}^{\mathrm{A}}=\mathrm{I}, \quad \Gamma_{2 \mathrm{~N}}^{5} \Gamma_{2 \mathrm{~N}}^{6}=i .
$$

The relationship (45) between the Clifford-Dirac algebra $\mathrm{Cl}^{\mathrm{R}}(0,6)$ and algebra $\mathrm{SO}(8)$ is similar to the relationship between the standard Clifford-Dirac algebra $\mathrm{Cl}^{\mathrm{C}}(1,3)$ and algebra $\mathrm{SO}(3,3)$ found in $[99,100]$; for the relationship between $\mathrm{Cl}^{\mathrm{C}}(1,3)$ and $\mathrm{SO}(1,5)$ see in [85-91].

Note that subalgebra $\mathrm{SO}(6) \subset \mathrm{SO}(8)$ is the algebra of invariance of the Dirac equation in the FW representation [6, 7]. The 15 elements of this $\mathrm{SO}(6)$ algebra are given by

$$
\left\{s^{\underline{A}} \underline{\mathrm{B}}\right\}, \quad \underline{\mathrm{A}}, \underline{\mathrm{B}}=\overline{1,6},
$$


where

$$
\left\{s^{\underline{\mathrm{A}}} \underline{\mathrm{B}}\right\}=\left\{s^{\underline{\mathrm{A}}} \underline{\mathrm{B}} \equiv \frac{1}{4}\left[\Gamma_{2 \mathrm{~N}}^{\mathrm{A}}, \Gamma_{2 \mathrm{~N}}^{\underline{\mathrm{B}}}\right]\right\} .
$$

Now only the first 6 matrices

$$
\left\{\Gamma_{2 \mathrm{~N}}^{\mathrm{A}}\right\}=\left\{\Gamma_{2 \mathrm{~N}}^{1}, \Gamma_{2 \mathrm{~N}}^{2}, \Gamma_{2 \mathrm{~N}}^{3}, \Gamma_{2 \mathrm{~N}}^{4}, \Gamma_{2 \mathrm{~N}}^{5}, \Gamma_{2 \mathrm{~N}}^{6}\right\}
$$

from the set (43) play the role of generating operators in the constructions (50), (51).

Here $\Gamma_{2 \mathrm{~N}}^{4}$ is independent. Therefore, in application to Clifford algebras operators (51) generate the 64dimensional $\mathrm{Cl}^{\mathrm{R}}(0,6)$ algebra as well.

The maximal pure matrix algebra of invariance of the FW equation (34) is the 31-dimensional algebra $\mathrm{SO}(6) \oplus i \Gamma_{2 \mathrm{~N}}^{0} \cdot \mathrm{SO}(6) \oplus i \Gamma_{2 \mathrm{~N}}^{0}$.

The additional possibilities, which are open by the 28 orts of the algebra $\mathrm{SO}(8)$ in comparison with 15 orts of the well-known algebra $\mathrm{SO}(1,5)$, are principal in description of the Bose states in the framework of the Dirac theory [85-91]. The algebra $\mathrm{SO}(8)$ includes two independent spin $\mathrm{s}=1 / 2 \mathrm{SU}(2)$ subalgebras $\left(s^{1} \equiv \frac{1}{2} \Gamma_{2 \mathrm{~N}}^{2} \Gamma_{2 \mathrm{~N}}^{3}, s^{2} \equiv\right.$ $\left.\frac{1}{2} \Gamma_{2 \mathrm{~N}}^{3} \Gamma_{2 \mathrm{~N}}^{1}, s^{3} \equiv \frac{1}{2} \Gamma_{2 \mathrm{~N}}^{1} \Gamma_{2 \mathrm{~N}}^{2}\right)$ and $\left(\check{s}^{1} \equiv \frac{1}{2} \Gamma_{2 \mathrm{~N}}^{5} \Gamma_{2 \mathrm{~N}}^{6}, \check{s}^{2} \equiv\right.$ $\frac{1}{2} \Gamma_{2 \mathrm{~N}}^{6} \Gamma_{2 \mathrm{~N}}^{4}, \check{s}^{3} \equiv \frac{1}{2} \Gamma_{2 \mathrm{~N}}^{4} \Gamma_{2 \mathrm{~N}}^{5}$ ) (in the anti-Hermitian form), whereas the $\mathrm{SO}(1,5)$ algebra includes only one set of $\mathrm{SU}(2)$ generators given by the elements $\left(s^{1} \equiv \frac{1}{2} \Gamma_{2 \mathrm{~N}}^{2} \Gamma_{2 \mathrm{~N}}^{3}, s^{2} \equiv\right.$ $\left.\frac{1}{2} \Gamma_{2 \mathrm{~N}}^{3} \Gamma_{2 \mathrm{~N}}^{1}, s^{3} \equiv \frac{1}{2} \Gamma_{2 \mathrm{~N}}^{1} \Gamma_{2 \mathrm{~N}}^{2}\right)$. Moreover, the algebra $\mathrm{SO}(6)$, which is the algebra of invariance of the FW equation (34), includes these two independent $\mathrm{SU}(2)$ subalgebras as well. As long as these two spin $s=1 / 2 \mathrm{SU}(2)$ sets of generators commute between each other, their combination gives the generators of spin $\mathrm{s}=1$ representation of $\mathrm{SU}(2)$ algebra. Such SU(2) algebra is the building element in construction of spin s=1 Lorentz and Poincaré algebras, with respect to which the FW equation (34) is invariant.

The transition to the RCQM is given by the transformation (30), (31). Thus, in the quantum-mechanical representation the seven $\Gamma$ matrices (43) (in the terms of standard $\Gamma_{2 \mathrm{~N}}^{\bar{\mu}}$ matrices (34), (35)) have the form (38) together with

$$
\begin{gathered}
\bar{\Gamma}_{2 \mathrm{~N}}^{5} \equiv \Gamma_{2 \mathrm{~N}}^{1} \Gamma_{2 \mathrm{~N}}^{3} \widehat{C}, \quad \bar{\Gamma}_{2 \mathrm{~N}}^{6} \equiv-i \Gamma_{2 \mathrm{~N}}^{2} \Gamma_{2 \mathrm{~N}}^{4} \widehat{C}, \quad \bar{\Gamma}_{2 \mathrm{~N}}^{7} \equiv i ; \\
\bar{\Gamma}_{2 \mathrm{~N}}^{1} \bar{\Gamma}_{2 \mathrm{~N}}^{2} \bar{\Gamma}_{2 \mathrm{~N}}^{3} \bar{\Gamma}_{2 \mathrm{~N}}^{4} \bar{\Gamma}_{2 \mathrm{~N}}^{5} \bar{\Gamma}_{2 \mathrm{~N}}^{6} \bar{\Gamma}_{2 \mathrm{~N}}^{7}=\mathrm{I}_{2 \mathrm{~N}}
\end{gathered}
$$

and satisfy the anti-commutation relations of the CliffordDirac algebra $\mathrm{Cl}^{\mathrm{R}}(0,6)$ representation in the following form

$$
\bar{\Gamma}_{2 \mathrm{~N}}^{\mathrm{A}} \bar{\Gamma}_{2 \mathrm{~N}}^{\mathrm{B}}+\bar{\Gamma}_{2 \mathrm{~N}}^{\mathrm{B}} \bar{\Gamma}_{2 \mathrm{~N}}^{\mathrm{A}}=-2 \delta^{\mathrm{AB}}, \quad \mathrm{A}, \mathrm{B}=\overline{1,7} .
$$

The RCQM representation of the algebra $\mathrm{SO}(8)$ is given by

$$
\bar{s}^{\overline{\mathrm{A}} \overline{\mathrm{B}}}=\left\{\bar{s}^{\mathrm{AB}}=\frac{1}{4}\left[\bar{\Gamma}_{2 \mathrm{~N}}^{\mathrm{A}}, \bar{\Gamma}_{2 \mathrm{~N}}^{\mathrm{B}}\right], \bar{s}^{\mathrm{A} 8}=-\bar{s}^{8 \mathrm{~A}}=\frac{1}{2} \bar{\Gamma}_{2 \mathrm{~N}}^{\mathrm{A}}\right\},
$$

$$
\overline{\mathrm{A}}, \overline{\mathrm{B}}=\overline{1,8},
$$

where the matrices $\bar{\Gamma}_{2 \mathrm{~N}}^{\mathrm{A}}$ are given in (38), (52) and generators $\bar{s} \overline{\mathrm{A}}^{\overline{\mathrm{A}} \mathrm{B}}$ satisfy the commutation relations

$$
\left[\bar{s}^{\overline{\mathrm{A}} \overline{\mathrm{B}}}, \bar{s}^{\overline{\mathrm{C}} \overline{\mathrm{D}}}\right]=\delta^{\overline{\mathrm{A}} \overline{\mathrm{C}}_{\bar{s}} \overline{\mathrm{B}} \overline{\mathrm{D}}}+\delta^{\overline{\mathrm{C}} \overline{\mathrm{B}}} \bar{s}^{\overline{\mathrm{D}} \overline{\mathrm{A}}}+\delta^{\overline{\mathrm{B}} \overline{\mathrm{D}}} \bar{s}^{\overline{\mathrm{A}} \overline{\mathrm{C}}}+\delta^{\overline{\mathrm{D}} \overline{\mathrm{A}}} \overline{\overline{\mathrm{C}}} \overline{\mathrm{C}} .
$$

The RCQM representation of the algebra $\mathrm{SO}(6) \subset \mathrm{SO}(8)$ is given by

$$
\left\{\bar{s}^{\underline{\mathrm{A}}} \underline{\mathrm{B}}\right\}, \quad \underline{\mathrm{A}}, \underline{\mathrm{B}}=\overline{1,6},
$$

where

$$
\left\{\bar{s}^{\underline{\mathrm{A}}} \underline{\mathrm{B}}\right\}=\left\{\bar{s}^{\underline{\mathrm{A}}} \underline{\mathrm{B}} \equiv \frac{1}{4}\left[\bar{\Gamma}^{\frac{\mathrm{A}}{2 \mathrm{~N}}}, \bar{\Gamma}_{\frac{\mathrm{B}}{2 \mathrm{~N}}}\right]\right\} .
$$

The maximal pure matrix algebra of invariance of the Schrödinger-Foldy equation (13) is the 31-dimensional algebra $\mathrm{a}_{31}=\mathrm{SO}(6) \oplus i \cdot \mathrm{SO}(6) \oplus i$.

In the RCQM representation two independent sets of generators of $\mathrm{SU}(2)$ subalgebra of $\mathrm{SO}(6)$ algebra, with respect to which the Schrödinger-Foldy equation (13) is invariant, have the form

$$
\begin{gathered}
\bar{s}^{1} \equiv \frac{1}{2} \bar{\Gamma}_{2 \mathrm{~N}}^{2} \bar{\Gamma}_{2 \mathrm{~N}}^{3}, \bar{s}^{2} \equiv \frac{1}{2} \bar{\Gamma}_{2 \mathrm{~N}}^{3} \bar{\Gamma}_{2 \mathrm{~N}}^{1}, \bar{s}^{3} \equiv \frac{1}{2} \bar{\Gamma}_{2 \mathrm{~N}}^{1} \bar{\Gamma}_{2 \mathrm{~N}}^{2}, \\
\bar{s}^{1} \equiv \frac{1}{2} \bar{\Gamma}_{2 \mathrm{~N}}^{5} \bar{\Gamma}_{2 \mathrm{~N}}^{6}, \bar{s}^{2} \equiv \frac{1}{2} \bar{\Gamma}_{2 \mathrm{~N}}^{6} \bar{\Gamma}_{2 \mathrm{~N}}^{4}, \bar{s} \equiv \frac{1}{2} \bar{\Gamma}_{2 \mathrm{~N}}^{4} \bar{\Gamma}_{2 \mathrm{~N}}^{5},
\end{gathered}
$$

where the matrices $\bar{\Gamma}_{2 \mathrm{~N}}^{\mathrm{A}}$ are given in (38), (52). The situation here is similar to the FW representation. As long as these two spin $s=1 / 2 \mathrm{SU}(2)$ sets of generators commute between each other, their combination gives the generators of spin $s=1$ representation of $S U(2)$ algebra. Such $S U(2)$ algebra is the building element in construction of spin $s=1$ Lorentz and Poincaré algebras, with respect to which the Schrödinger-Foldy equation (13) is invariant. Note that formulas (58), (59) are valid for the N-dimensional multiplet but only for the spin $s=1 / 2$. The arbitrary spin cannot be written in terms of $\Gamma_{2 \mathrm{~N}}$ matrices as (58), (59).

The partial case of 4 component formalism and $4 \times 4 \gamma$ matrices algebra follows from the above given consideration after corresponding substitutions $2 \mathrm{~N}=4$, etc. The improved consideration of this axiom in [1] for the spin $1 / 2$ particle-antiparticle doublet should be taken from the text above as the corresponding particular case.

An important fact is that in Pauli-Dirac representation the 31-dimensional algebra $\mathrm{SO}(6) \oplus i \Gamma_{2 \mathrm{~N}}^{0} \cdot \mathrm{SO}(6) \oplus i \Gamma_{2 \mathrm{~N}}^{0}$ still is the algebra of invariance of the Dirac equation (or $2 \mathrm{~N}$ component Dirac-like equation). The only difference is that in Pauli-Dirac representation the operators $s^{\underline{A}} \underline{\mathrm{B}}$ and gamma matrices $\Gamma_{2 \mathrm{~N}}^{\mathrm{A}}$ are not pure matrix. They are the nonlocal pseudo-differential operators, which contain the operator $\widehat{\omega} \equiv \sqrt{-\Delta+m^{2}}$. For the standard 4 component Dirac equation the corresponding gamma matrices are given by (18)-(22) in [91].

Another interesting fact is that operator of the Dirac equation with Hamiltonian $H \equiv \gamma^{0} \vec{\gamma} \cdot \vec{p}+\gamma^{0} m-e^{2} /|\vec{x}|$ commutes with all 31 generators of the $\mathrm{SO}(6) \oplus i \gamma^{0}$. $\mathrm{SO}(6) \oplus i \gamma^{0}$ algebra, which elements are given in terms of gamma matrices from (18)-(22) in [91]. Therefore, the relativistic hydrogen atom has wide additional symmetries given by the 31 nontrivial generators from $\mathrm{SO}(6) \oplus i \gamma^{0}$. $\mathrm{SO}(6) \oplus i \gamma^{0}$. Moreover, the relativistic hydrogen atom has additional spin 1 symmetries, which for the free Dirac 
equation are known from [85-91] and which are the consequences of the algebra $\mathrm{SO}(6) \oplus i \gamma^{0} \cdot \mathrm{SO}(6) \oplus i \gamma^{0}$. Indeed, more wide symmetries than $\mathrm{SO}(4)$ spin $1 / 2$ symmetry of $\mathrm{V}$. Fock [101] or further symmetries found in [102-106] (see the brief analysis in [107]) can be found on the basis of this 31-dimensional algebra.

Note that consideration of this axiom above is given as the simple generalization of the correct spin $1 / 2$ particleantiparticle doublet formalism. In reality the dimensions of Clifford-Dirac (and SO(n)) algebras for the higher spin cases $2(2 s+1) \geq 8$ can be more higher then considered above 64-dimensional $\mathrm{Cl}^{\mathrm{R}}(4,2)$ and $\mathrm{Cl}^{\mathrm{R}}(0,6)$ algebras (and $\mathrm{SO}(8)$ algebra). The reason of this situation is the possibility to construct for $2(2 s+1) \geq 8$ cases the additional $\Gamma$ matrices obeying the Clifford-Dirac anticommutation relations. The concrete examples of such additional $\Gamma$ matrices can be found in [108, 109].

Visual and demonstrative description of matrix representations of $\mathrm{Cl}^{\mathrm{R}}(0,6)$ and $\mathrm{SO}(8)$ algebras in terms of $4 \times 4$ gamma matrices can be found in [110].

\subsection{On the main and additional conservation laws}

Similarly to the nonrelativistic quantum mechanics the conservation laws are found in the form of quantummechanical mean values of the operators, which commute with the operator of the equation of motion.

The important physical consequence of the assertion about the relativistic invariance is the fact that 10 integral dynamical variables of the doublet

$$
\left(P_{\mu}, J_{\mu \nu}\right) \equiv \int d^{3} x f^{\dagger}(t, \vec{x})\left(\widehat{p}_{\mu}, \widehat{j}_{\mu \nu}\right) f(t, \vec{x})=\text { Const }
$$

do not depend on time, i. e. they are the constants of motion for this doublet. In (60) and below in the text of this axiom the wave function is chosen as (4), (14) and generators $\left(\widehat{p}_{\mu}, \widehat{j}_{\mu \nu}\right)$ are given in (22), (23)].

Note that the external and internal degrees of freedom for the free arbitrary spin particle-antiparticle doublet are independent. Therefore, the operator $\vec{s}$ (15) commutes not only with the operators $\widehat{\vec{p}}, \vec{x}$, but also with the orbital part $\widehat{m}_{\mu \nu}$ of the total angular momentum operator. And both operators $\vec{s}$ and $\widehat{m}_{\mu \nu}$ commute with the operator $i \partial_{t}-\sqrt{-\Delta+m^{2}}$ of the equation (13). Therefore, besides the 10 main (consequences of the 10 Poincaré generators) conservation laws (60), 12 additional constants of motion exist for the free arbitrary spin particle-antiparticle doublet. These additional conservation laws are the consequences of the operators of the following observables (index $2 \mathrm{~N}$ is omitted):

$$
\begin{gathered}
s_{j}, \quad \breve{s}_{\ell}=\frac{s_{\ell n} \widehat{p}_{n}}{\widehat{\omega}+m}, \quad \widehat{m}_{\ell n}=x_{l} \widehat{p}_{n}-x_{n} \widehat{p}_{\ell}, \\
\widehat{m}_{0 \ell}=-\widehat{m}_{l 0}=t \widehat{p}_{\ell}-\frac{1}{2}\left\{x_{\ell}, \widehat{\omega}\right\},
\end{gathered}
$$

part of which are given and explained in (27). Here $s_{j}=$ $s_{\ell n}$ are given in (15).

Thus, the following assertions can be proved. In the space $\mathrm{H}^{\mathrm{A}}=\{A\}$ of the quantum-mechanical amplitudes the 10 main conservation laws (60) have the form

$$
\begin{gathered}
\left(P_{\mu}, J_{\mu \nu}\right)=\int d^{3} k A^{\dagger}(\vec{k})\left(\check{\widetilde{p}}_{\mu}, \check{\widetilde{j}}_{\mu \nu}\right) A(\vec{k}), \\
A(\vec{k}) \equiv\left|\begin{array}{c}
a^{1}(\vec{k}) \\
a^{2}(\vec{k}) \\
\cdot \\
\cdot \\
a^{2 \mathrm{~N}}(\vec{k})
\end{array}\right|,
\end{gathered}
$$

where the density generators of $\mathcal{P}^{\mathrm{A}},\left(\check{\widetilde{p}}_{\mu}, \check{\widetilde{j}}_{\mu \nu}\right)$ from (62) are given by

$$
\begin{aligned}
& \check{\widetilde{p}}_{0}=p_{0}=\omega, \check{\widetilde{p}}_{\ell}=p_{\ell}=k_{\ell}, \check{\widetilde{j}}_{\ell n}=\widetilde{x}_{\ell} k_{n}-\widetilde{x}_{n} k_{\ell}+s_{\ell n}, \\
& \check{\widetilde{j}}_{0 \ell}=-\frac{1}{2}\left\{\widetilde{x}_{\ell}, \omega\right\}-\left(\breve{\widetilde{s}}_{\ell} \equiv \frac{s_{\ell n} k_{n}}{\omega+m}\right) ; \quad\left(\widetilde{x}_{l}=-i \frac{\partial}{\partial k^{l}}\right) .
\end{aligned}
$$

In the formula (62) $A(\vec{k})$ is a $2 \mathrm{~N}$-column of amplitudes.

Note that the operators (62)-(64) satisfy the Poincaré commutation relations in the manifestly covariant form (21).

It is evident that the 12 additional conservation laws

$$
\left(M_{\mu \nu}, S_{\ell n}, \breve{S}_{\ell}\right) \equiv \int d^{3} x f^{\dagger}(t, \vec{x})\left(\widehat{m}_{\mu \nu}, s_{\ell n}, \breve{s}_{\ell}\right) f(t, \vec{x})
$$

generated by the operators (61), are the separate terms in the expressions (62)-(64) of principal (main) conservation laws.

The 22 above given conservation laws are valid for an arbitrary spin (in the case $s=0$ we have only 10 conservation laws generated by operators (27)).

Additional set of conservation laws is given by 31 quantities

$$
\left(\mathrm{A}_{31}\right) \equiv \int d^{3} x f^{\dagger}(t, \vec{x})\left(\widehat{\mathrm{a}}_{31}\right) f(t, \vec{x})=\text { Const },
$$

or in terms of quantum-mechanical amplitudes

$$
\left(\mathrm{A}_{31}\right)=\int d^{3} k A^{\dagger}(\vec{k})\left(\widehat{\mathrm{a}}_{31}\right) A(\vec{k}) .
$$

Here $\left(\widehat{a}_{31}\right)$ are the pure matrix generators of the algebra $\mathrm{a}_{31}=\mathrm{SO}(6) \oplus i \cdot \mathrm{SO}(6) \oplus i$.

The conservation law of charge for the singlet has the form $q_{\mathrm{s}}=e \int d^{3} x f^{\dagger}(t, \vec{x}) f(t, \vec{x})$ and in the case of particle-antiparticle doublet this quantity is given by $q_{\mathrm{d}}=e \int d^{3} x f^{\dagger}(t, \vec{x}) g f(t, \vec{x})$, where operator $\mathrm{g}$ is presented in (15). In terms of quantum-mechanical amplitudes the corresponding conservation laws are given by $q_{\mathrm{s}}=\int d^{3} k A^{\dagger}(\vec{k}) A(\vec{k}), q_{\mathrm{d}}=\int d^{3} k A^{\dagger}(\vec{k}) g A(\vec{k})$.

In the case of spin $\mathrm{s}=1 / 2$ the conservation law of spin is given twice: in the set (65) and in the set (67). For cases s $>1 / 2$ the sets (65) and (67) are completely different. 


\subsection{On the stationary complete sets of ope- rators}

Let us consider now the outstanding role of the different complete sets of operators from the algebra of observables $\mathrm{A}_{\mathrm{S}}$. If one does not appeal to the complete sets of operators, then the solutions of the the Schrödinger-Foldy equation (13) are linked directly only with the Sturm-Liouville problem for the energy operator (5). In this case one comes to so-called "degeneration" of solutions. Recall that for an arbitrary complete sets of operators the notion of degeneration is absent in the Sturm-Liouville problem (see, e.g., [80]): only one state vector corresponds to any one point of the common spectrum of a complete set of operators. To wit, for a complete set of operators there is a one to one correspondence between any point of the common spectrum and an eigenvector.

The stationary complete sets play the special role among the complete sets of operators. Recall that the stationary complete set is the set of all functionally independent mutually commuting operators, each of which commutes with the operator of energy (in our case with the operator (5)). The examples of the stationary complete sets in $\mathrm{H}^{3,2 \mathrm{~N}}$ are given by $\left(\widehat{\vec{p}}, s_{z} \equiv s_{2 \mathrm{~N}}^{3}, g\right),\left(\vec{p}, \vec{s}_{2 \mathrm{~N}} \cdot \vec{p}, g\right)$, ets; $g$ is the charge sign operator. The set $\left(\vec{x}, s_{z}, g\right)$ is an example of non-stationary complete set. The $\vec{x}$-realization (10) of the space $\mathrm{H}^{3,2 \mathrm{~N}}$ and of quantum-mechanical SchrödingerFoldy equation (13) are related just to this complete set.

For the goals of this paper the stationary complete set $\left(\widehat{\vec{p}}, s_{z} \equiv s_{2 \mathrm{~N}}^{3}, g\right)$ is chosen. The series of partial examples of equations on eigenvectors and eigenvalues for this stationary complete set is given in [1]. Consider here as an example the spin $s=3 / 2$ particle-antiparticle doublet case. Corresponding equations on eigenvectors and eigenvalues are given by

$$
\begin{gathered}
\widehat{\vec{p}} e^{-i k x} \mathrm{~d}_{\overline{\mathrm{A}}}=\vec{k} e^{-i k x} \mathrm{~d}_{\overline{\mathrm{A}}}, \quad \overline{\mathrm{A}}=\overline{1,8}, \\
s_{8}^{3} \mathrm{~d}_{1}=\frac{3}{2} \mathrm{~d}_{1}, s_{8}^{3} \mathrm{~d}_{2}=\frac{1}{2} \mathrm{~d}_{2}, s_{8}^{3} \mathrm{~d}_{3}=-\frac{1}{2} \mathrm{~d}_{3}, s_{8}^{3} \mathrm{~d}_{4}=-\frac{3}{2} \mathrm{~d}_{4}, \\
s_{8}^{3} \mathrm{~d}_{5}=-\frac{3}{2} \mathrm{~d}_{5}, s_{8}^{3} \mathrm{~d}_{6}=-\frac{1}{2} \mathrm{~d}_{6}, s_{8}^{3} \mathrm{~d}_{7}=\frac{1}{2} \mathrm{~d}_{7}, s_{8}^{3} \mathrm{~d}_{8}=\frac{3}{2} \mathrm{~d}_{8}, \\
g \mathrm{~d}_{1}=-\mathrm{d}_{1}, g \mathrm{~d}_{2}=-\mathrm{d}_{2}, g \mathrm{~d}_{3}=-\mathrm{d}_{3}, g \mathrm{~d}_{4}=-\mathrm{d}_{4}, \quad(70) \\
g \mathrm{~d}_{5}=+\mathrm{d}_{5}, g \mathrm{~d}_{6}=+\mathrm{d}_{6}, g \mathrm{~d}_{7}=+\mathrm{d}_{7}, g \mathrm{~d}_{8}=+\mathrm{d}_{8},
\end{gathered}
$$

where the Cartesian orts $\left\{\mathrm{d}_{\overline{\mathrm{A}}}\right\}$ are given by $\left\{\mathrm{d}_{\overline{\mathrm{A}}}\right\}=$ $\left\{\delta_{\overline{\mathrm{A}}}^{\overline{\mathrm{B}}}\right\}, \overline{\mathrm{A}}, \overline{\mathrm{B}}=\overline{1,8}$.

Comparison of the given in equations (69) spin $s=1 / 2$ case with the spin $s=2$ case ((194) in [1]) shows that the general form is out of clear visualization. Thus, the appealing to such general form is absent here.

The interpretation of the amplitudes in the general solution (14) follows from equations of (68)-(70) type for the fixed value of arbitrary spin. In general, the functions $a^{2 \mathrm{~N}}(\vec{k})$ are the quantum-mechanical momentum-spin amplitudes. Thus, the first half of functions $a^{2 \mathrm{~N}}(\vec{k})$ is the momentum-spin amplitudes of the particle with the momentum $\widehat{\vec{p}}$, sign of the charge (-) and corresponding spin projections, respectively. Further, the second (bottom) half of functions $a^{2 \mathrm{~N}}(\vec{k})$ is the momentum-spin amplitudes of the antiparticle with the momentum $\widehat{\vec{p}}$, sign of the charge $(+)$ and opposite spin projections, respectively.

Thus, the conclusion about the fermionic (or bosonic) spin features of the solution (14) (i. e. the interpretation of the solution (14)) follows from the equations on eigenvectors and eigenvalues (of (68)-(70) type) and the above given interpretation of the amplitudes.

\subsection{On the solutions of the Schrödinger- Foldy equation}

Let us consider the Schrödinger-Foldy equation (13) general solution related to the stationary complete sets $\left(\widehat{\vec{p}}, s_{z} \equiv s^{3}, g\right)$, where $s^{3}$ is given in (15). The fundamental solutions of the equation (13), which are the eigen solutions of this stationary complete sets, are given by the relativistic de Broglie waves:

$$
\varphi_{\vec{k} 2 \mathrm{~N}}(t, \vec{x})=\frac{1}{(2 \pi)^{\frac{3}{2}}} e^{-i \omega t+i \vec{k} \vec{x}} \mathrm{~d}_{2 \mathrm{~N}},
$$

where $\mathrm{d}_{2 \mathrm{~N}}$ are the orts of $2 \mathrm{~N}$-dimensional Cartesian basis (the Cartesian orts are the common eigenvectors for the operators $\left.\left(s_{z}, g\right)\right)$. The explicit form of $\mathrm{N}$-dimensional Cartesian basis is given in formulas (10) of [1].

Vectors (71) are the generalized solutions of the equation (13). These solutions do not belong to the quantummechanical space $\mathrm{H}^{3,2 \mathrm{~N}}$, i. e. they are not realized in the nature. Nevertheless, the solutions (71) are the complete ortonormalized orts in the rigged Hilbert space $\mathrm{S}^{3,2 \mathrm{~N}} \subset$ $\mathrm{H}^{3,2 \mathrm{~N}} \subset \mathrm{S}^{3,2 \mathrm{~N} *}$. In symbolic form the conditions of ortonormalization and completeness are given by

$$
\begin{aligned}
& \int d^{3} x \varphi_{\vec{k} \alpha}^{\dagger}(t, \vec{x}) \varphi_{\vec{k}^{\prime} \alpha^{\prime}}(t, \vec{x})=\delta\left(\vec{k}-\vec{k}^{\prime}\right) \delta_{\alpha \alpha^{\prime}}, \\
& \int d^{3} k \sum_{\alpha=1}^{2 \mathrm{~N}} \varphi_{\vec{k} \alpha}^{\beta}(t, \vec{x}) \varphi_{\vec{k} \alpha}^{* \beta^{\prime}}\left(t, \vec{x}^{\prime}\right)=\delta\left(\vec{x}-\vec{x}^{\prime}\right) \delta_{\beta \beta^{\prime}}
\end{aligned}
$$

The functional forms of these conditions are omitted because of their bulkiness.

In the rigged Hilbert space $\mathrm{S}^{3,2 \mathrm{~N}} \subset \mathrm{H}^{3,2 \mathrm{~N}} \subset \mathrm{S}^{3,2 \mathrm{~N} *}$ an arbitrary solution of the equation (13) can be decomposed in terms of fundamental solutions (71). Furthermore, for the solutions $f \in \mathrm{S}^{3,2 \mathrm{~N}} \subset \mathrm{H}^{3,2 \mathrm{~N}}$ the expansion (14) is, (i) mathematically well-defined in the framework of the standard differential and integral calculus, (ii) if in the expansion (14) a state $f \in \mathrm{S}^{3,2 \mathrm{~N}} \subset \mathrm{H}^{3,2 \mathrm{~N}}$, then the amplitudes $a^{2 \mathrm{~N}}(\vec{k})$ in (14) belong to the set of the Schwartz test functions over $\mathrm{R}_{\vec{k}}^{3}$. Therefore, they have the unambiguous physical sense of the amplitudes of probability distributions over the eigenvalues of the stationary complete 
sets $\left(\widehat{\vec{p}}, s_{z}, g\right)$. Moreover, the complete set of quantummechanical amplitudes unambiguously determine the corresponding representation of the space $\mathrm{H}^{3,2 \mathrm{~N}}$ (in this case it is the $\left(\vec{k}, s_{z}, g\right)$-representation), which vectors have the harmonic time dependence

$$
\widetilde{f}(t, \vec{k})=e^{-i \omega t} A(\vec{k}), \quad A(\vec{k}) \equiv\left|\begin{array}{c}
a^{1}(\vec{k}) \\
a^{2}(\vec{k}) \\
\cdot \\
\cdot \\
a^{2 \mathrm{~N}}(\vec{k})
\end{array}\right|,
$$

i. e. are the states with the positive sign of the energy $\omega$.

The similar assertion is valid for the expansions of the states $f \in \mathrm{H}^{3,2 \mathrm{~N}}$ over the basis states, which are the eigenvectors of an arbitrary stationary complete sets. Therefore, the corresponding representation of the space $\mathrm{H}^{3,2 \mathrm{~N}}$, which is related to such expansions, is often called as the generalized Fourier transformation.

By the way, the $\vec{x}$-realization (10) of the states space is associated with the non-stationary complete set of operators $\left(\vec{x}, s_{z}, g\right)$. Therefore, the amplitudes $f^{2 \mathrm{~N}}(t, \vec{x})=$ $\mathrm{d}_{2 \mathrm{~N}}^{\dagger} f(t, \vec{x})=U(t) f(0, \vec{x})$ of the probability distribution over the eigenvalues of this complete set depend on time $t$ non-harmonically.

\subsection{The axiom on the mean value of the ope- rators of observables}

Note that any apparatus can not fulfill the absolutely precise measurement of a value of the physical quantity having continuous spectrum. Therefore, the customary quantummechanical axiom about the possibility of "precise" measurement, for example, of the coordinate (or another quantity with the continuous spectrum), which is usually associated with the corresponding "reduction" of the wave-packet, can be revisited. This assertion for the values with the continuous spectrum can be replaced by the axiom that only the mean value of the operator of observable (or the corresponding complete set of observables) is the experimentally observed for $\forall f \in \mathrm{H}^{3, \mathrm{~N}}$. Such axiom, without any loss of generality of consideration, unambiguously justifies the using of the subspace $\mathrm{S}^{3, \mathrm{~N}} \subset \mathrm{H}^{3, \mathrm{~N}}$ as an approximative space of the physically realizable states of the considered object. This axiom as well does not enforce the application of the conception of the ray in $\mathrm{H}^{3, \mathrm{~N}}$ (the set of the vectors $e^{i \alpha} f$ with an arbitrary-fixed real number $\alpha$ ) as the state of the object. Therefore, the mapping $(a, \varpi) \rightarrow U(a, \varpi)$ in the formula (22) for the $\mathcal{P}$-representations in $\mathrm{S}^{3, \mathrm{~N}} \subset \mathrm{H}^{3, \mathrm{~N}}$ is an unambiguous. Such axiom actually removes the problem of the wave packet "reduction", which discussion started from the well-known von Neumann monograph [38]. Therefore, the subjects of the discussions of all "paradoxes" of quantum mechanics, a lot of attention to which was paid in the past century, are removed also.

The important conclusion about the RCQM is as follows. The consideration of all aspects of this model is given on the basis of using only such conceptions and quantities, which have the direct relation to the experimentally observable physical quantities of this "elementary" physical system.

\subsection{On the principles of heredity and the correspondence}

The explicit forms (60)-(65) of the main and additional conservation laws demonstrate evidently that the model of RCQM satisfies the principles of the heredity and the correspondence with the non-relativistic classical and quantum theories. The deep analogy between RCQM and these theories for the physical system with the finite number degrees of freedom (where the values of the free dynamical conserved quantities are additive) is also evident.

Our new way of the Dirac equation derivation (section 9 in [1]) has been started from the RCQM of the spin $\mathrm{s}=(1 / 2,1 / 2)$ particle-antiparticle doublet.

\subsection{On the second quantization}

This axiom is external (not internal) in the RCQM. It is necessary for quantum field theory. The formalism of RCQM is complete without this axiom. The reader can see the brief consideration in [1] (section 8) in the example of spin $\mathrm{s}=(1 / 2,1 / 2)$ particle-antiparticle doublet.

\subsection{On the physical interpretation}

The physical interpretation always is the final step in the arbitrary model of the physical reality formulation. In the beginning of the interpretation is better to recall the different formulations of the quantum theory collected, e. g., in [111].

The above considered model of physical reality is called the canonical quantum mechanics due to the principles of the heredity and correspondence with nonrelativistic Schrödinger quantum mechanics [38].

The above considered canonical quantum mechanics is called the relativistic canonical quantum mechanics due to its invariance with respect to the corresponding representations of the Poincare group $\mathcal{P}$.

The above considered RCQM describes the spin $(\mathrm{s}, \mathrm{s})$ particle-antiparticle doublet due to the corresponding eigenvalues in the equations like (68)-(70) for the stationary complete set of operators and the explicit forms of the $\mathrm{Ca}$ simir operators (25), (26) of the corresponding Poincaré group $\mathcal{P}$ representation, with respect to which the dynamical equation of motion is invariant.

The axioms of this section eventually need to be reconciled with three levels of description used in this paper: RCQM, canonical FW and Dirac models. Nevertheless, this interesting problem cannot be considered in few pages. The readers of this paper can compare the axioms of RCQM given above with the main principles of the Dirac 
model given in B. Thaller's monograph [112] on the high mathematical level.

\section{General description of the arbi- trary spin field theory}

The step by step consideration of the different partial examples in [1] (sections 21-27) enabled us to rewrite them in the general form, which is valid for arbitrary spin. The mathematical induction is applied. Therefore, the generalization of the consideration given in [1] leads to the general formalism of the arbitrary spin fields.

The formalism presented below in this section is valid for an arbitrary particle-antiparticle multiplet in general and for the particle-antiparticle doublet of fixed value of spin in particular.

\subsection{The canonical (FW type) model of the ar- bitrary spin particle-antiparticle field}

The operator, which transform the RCQM of the arbitrary spin particle-antiparticle multiplet into the corresponding canonical particle-antiparticle field, is given by (30). As it is explained already in section 6 above (axiom on the Clifford-Dirac algebra) the transition with the help of the operator (30) is possible for the anti-Hermitian operators.

The formulas mentioned below are found from the corresponding formulas of RCQM with the help of the operator (30) on the basis of its properties (31), (32). For the general form of arbitrary spin canonical particle-antiparticle field the equation of motion of the FW type is given by (34). The general solution has the form (33), where $a^{\mathrm{N}}(\vec{k})$ are the quantum-mechanical momentum-spin amplitudes of the particle and $a^{\breve{N}}(\vec{k})$ are the quantum-mechanical momentum-spin amplitudes of the antiparticle, $\{\mathrm{d}\}$ is $2 \mathrm{~N}$ component Cartesian basis.

It is evident from (33) that the model under consideration is not quantum mechanics. Indeed, contrary to (14) the solution (33) contains positive and negative frequency terms and, as a consequence, equation (34) is dealing with positive and negative energies (contrary to equation (13)).

The spin operator, which follows from (15), has the form

$$
\vec{s}_{2 \mathrm{~N}}=\left|\begin{array}{ll}
\vec{s}_{\mathrm{N}} & 0 \\
0 & \vec{s}_{\mathrm{N}}
\end{array}\right|, \quad \mathrm{N}=2 s+1,
$$

where $\vec{s}_{\mathrm{N}}$ are $\mathrm{N} \times \mathrm{N}$ generators of arbitrary spin irreducible representations of $\mathrm{SU}(2)$ algebra, which satisfy the commutation relations (18). In the terms of $\Gamma_{2 \mathrm{~N}}$ matrices (34), (35) the spin operator (75) has the form (40) (note that the form (40) is valid only in the dimensions where the gamma matrices exist).

The generators of the reducible unitary representation of the Poincare group $\mathcal{P}$, with respect to which the canonical field equation (34) and the set $\{\phi\}$ of its solutions (33) are invariant, are given by

$$
\begin{gathered}
\widehat{p}^{0}=\Gamma_{2 \mathrm{~N}}^{0} \widehat{\omega} \equiv \Gamma_{2 \mathrm{~N}}^{0} \sqrt{-\Delta+m^{2}}, \quad \widehat{p}^{\ell}=-i \partial_{\ell}, \quad(76) \\
\hat{j}^{\ell n}=x^{\ell} \widehat{p}^{n}-x^{n} \widehat{p}^{\ell}+s_{2 \mathrm{~N}}^{\ell n} \equiv \widehat{m}^{\ell n}+s_{2 \mathrm{~N}}^{\ell n}, \\
\widehat{j}^{\ell \ell}=-\hat{j}^{\ell 0}=x^{0} \widehat{p}^{\ell}-\frac{1}{2} \Gamma_{2 \mathrm{~N}}^{0}\left\{x^{\ell}, \widehat{\omega}\right\}+\Gamma_{2 \mathrm{~N}}^{0} \frac{\left(\vec{s}_{2 \mathrm{~N}} \times \vec{p}\right)^{\ell}}{\widehat{\omega}+m},
\end{gathered}
$$

where arbitrary spin $\mathrm{SU}(2)$ generators $\vec{s}_{2 \mathrm{~N}}=\left(s_{2 \mathrm{~N}}^{\ell n}\right)$ have the form (75), $\Gamma_{2 \mathrm{~N}}^{0}$ is given in (34).

Note that together with the generators (76), (77) another set of 10 operators commutes with the operator of equation (34), satisfies the commutation relations (21) of the Lie algebra of Poincaré group $\mathcal{P}$, and, therefore, can be chosen as the Poincaré symmetry of the model under consideration. This second set is given by the generators $\widehat{p}^{0}, \widehat{p}^{\ell}$ from (76) together with the orbital parts of the generators $\hat{j}^{\ell n}, \widehat{j}^{0 \ell}$ from (76), (77), respectively. In another way this set follows from the set (27) after the transformation (30), (31).

The calculation of the Casimir operators $p^{2}=$ $\widehat{p}^{\mu} \widehat{p}_{\mu}, W=w^{\mu} w_{\mu}\left(w^{\mu}\right.$ is the Pauli-Lubanski pseudovector) for the fixed value of spin completes the brief description of the model.

\subsection{The locally covariant model of the arbi- trary spin particle-antiparticle field}

The operator, which transform the canonical (FW type) model of the arbitrary spin particle-antiparticle field into the corresponding locally covariant particle-antiparticle field, is the generalized FW operator and is given by

$$
\begin{gathered}
V^{\mp}=\frac{\mp \vec{\Gamma}_{2 \mathrm{~N}} \cdot \vec{p}+\widehat{\omega}+m}{\sqrt{2 \widehat{\omega}(\widehat{\omega}+m)}}, \quad V^{-}=\left(V^{+}\right)^{\dagger}, \\
V^{-} V^{+}=V^{+} V^{-}=\mathrm{I}_{2 \mathrm{~N}}, \quad \mathrm{~N}=2 s+1,
\end{gathered}
$$

where $\Gamma_{2 \mathrm{~N}}^{j}$ are known from (35) and $\Sigma_{\mathrm{N}}^{j}$ are the $\mathrm{N} \times \mathrm{N}$ Pauli matrices.

Of course, for the matrices $\Gamma_{2 \mathrm{~N}}^{\mu}$ (34), (35) (together with the matrix $\Gamma_{2 \mathrm{~N}}^{4} \equiv \Gamma_{2 \mathrm{~N}}^{0} \Gamma_{2 \mathrm{~N}}^{1} \Gamma_{2 \mathrm{~N}}^{2} \Gamma_{2 \mathrm{~N}}^{3}$ ) the relations (29) are valid.

Note that in formulas (78) and in all formulas before the end of the subsection the values of $\mathrm{N}$ are only even. Therefore, the canonical field equation (34) describes the larger number of multiplets then the generalized Dirac equation (79) given below.

The formulas (79)-(84) below are found from the corresponding formulas (33), (34), (75)-(77) of canonical field model on the basis of the operator (78).

For the general form of arbitrary spin locally covariant particle-antiparticle field the Dirac-like equation of motion follows from the equation (34) after the transformation (78) and is given by

$$
\left[i \partial_{0}-\Gamma_{2 \mathrm{~N}}^{0}\left(\vec{\Gamma}_{2 \mathrm{~N}} \cdot \vec{p}+m\right)\right] \psi(x)=0 .
$$


The general solution has the form

$$
\begin{gathered}
\psi(x)=V^{-} \phi(x)= \\
\frac{1}{(2 \pi)^{\frac{3}{2}}} \int d^{3} k\left[e^{-i k x} a^{\mathrm{N}}(\vec{k}) \mathrm{v}_{\mathrm{N}}^{-}(\vec{k})+e^{i k x} a^{* \mathrm{~N}}(\vec{k}) \mathrm{v}_{\breve{\mathrm{N}}}^{+}(\vec{k})\right],
\end{gathered}
$$

where amplitudes and notation $\breve{N}$ are the same as in (33); $\left\{\mathrm{v}_{\mathrm{N}}^{-}(\vec{k}), \mathrm{v}_{\mathrm{N}}^{+}(\vec{k})\right\}$ are $2 \mathrm{~N}$-component Dirac basis spinors with properties of ortonormalization and completeness similar to 4-component Dirac spinors from [113].

The spin operator is given by

$$
\vec{s}_{\mathrm{D}}=V^{-} \vec{s}_{2 \mathrm{~N}} V^{+},
$$

where operator $\vec{s}_{2 \mathrm{~N}}$ is known from (75). The explicit forms of few partial cases of spin operators (81) are given in formulae (259)-(261), (284)-(286), (359) of [1] for the particle-antiparticle multiplets $s=(1,0,1,0), s=(3 / 2,3 / 2)$, $\mathrm{s}=(2,1,2,1)$, respectively.

The generators of the reducible unitary representation of the Poincaré group $\mathcal{P}$, with respect to which the covariant field equation (79) and the set $\{\psi\}$ of its solutions (80) are invariant, have the form

$$
\begin{gathered}
\widehat{p}^{0}=\Gamma_{2 \mathrm{~N}}^{0}\left(\vec{\Gamma}_{2 \mathrm{~N}} \cdot \vec{p}+m\right), \quad \widehat{p}^{\ell}=-i \partial_{\ell}, \\
\widehat{j}^{\ell n}=x_{\mathrm{D}}^{\ell} \widehat{p}^{n}-x_{\mathrm{D}}^{n} \widehat{p}^{\ell}+s_{\mathrm{D}}^{\ell n} \equiv \widehat{m}^{\ell n}+s_{\mathrm{D}}^{\ell n}, \\
\widehat{j}^{0 \ell}=-\widehat{j}^{\ell 0}=x^{0} \widehat{p}^{\ell}-\frac{1}{2}\left\{x_{\mathrm{D}}^{\ell}, \widehat{p}^{0}\right\}+\frac{\widehat{p}^{0}\left(\vec{s}_{\mathrm{D}} \times \vec{p}\right)^{\ell}}{\widehat{\omega}(\widehat{\omega}+m)},
\end{gathered}
$$

where the spin matrices $\vec{s}_{\mathrm{D}}=\left(s_{\mathrm{D}}^{\ell n}\right)$ are given in (81) and the operator $\vec{x}_{\mathrm{D}}$ has the form

$$
\vec{x}_{\mathrm{D}}=\vec{x}+\frac{i \vec{\Gamma}_{2 \mathrm{~N}}}{2 \widehat{\omega}}-\frac{\vec{s}_{2 \mathrm{~N}} \times \vec{p}}{\widehat{\omega}(\widehat{\omega}+m)}-\frac{i \vec{p}\left(\vec{\Gamma}_{2 \mathrm{~N}} \cdot \vec{p}\right)}{2 \widehat{\omega}^{2}(\widehat{\omega}+m)},
$$

where specific spin matrices $\vec{s}_{2 \mathrm{~N}}^{\Gamma}$ are given by

$$
\begin{gathered}
\vec{s}_{2 \mathrm{~N}}^{\Gamma} \equiv \vec{s}_{\mathrm{FW}}=\left(s_{2 \mathrm{~N}}^{1}, s_{2 \mathrm{~N}}^{2}, s_{2 \mathrm{~N}}^{3}\right)= \\
\quad \frac{i}{2}\left(\Gamma_{2 \mathrm{~N}}^{2} \Gamma_{2 \mathrm{~N}}^{3}, \Gamma_{2 \mathrm{~N}}^{3} \Gamma_{2 \mathrm{~N}}^{1}, \Gamma_{2 \mathrm{~N}}^{1} \Gamma_{2 \mathrm{~N}}^{2}\right) .
\end{gathered}
$$

Note that for corresponding partial cases of $\vec{x}_{\mathrm{D}}$ in [1] ( $(267)$ for $s=(1,0,1,0)$, once more $(267)$ but for $s=(3 / 2,3 / 2)$, (333) for $s=(2,0,2,0),(363)$ for $s=(2,1,2,1))$ the correspon$\operatorname{ding} \vec{s} \Gamma_{2 \mathrm{~N}}$ are given by

$$
\begin{gathered}
\mathrm{s}=(1,0,1,0) \text { and } \mathrm{s}=(3 / 2,3 / 2): \\
\vec{s}_{8} \equiv\left(s_{8}^{1}, s_{8}^{2}, s_{8}^{3}\right)=\frac{i}{2}\left(\Gamma_{8}^{2} \Gamma_{8}^{3}, \Gamma_{8}^{3} \Gamma_{8}^{1}, \Gamma_{8}^{1} \Gamma_{8}^{2}\right),
\end{gathered}
$$

for $\vec{x}_{\mathrm{D}}$ [1] (267), where the $\Gamma_{8}^{j}$ matrices are given in (253),

$$
\begin{gathered}
\mathrm{s}=(2,0,2,0): \\
\vec{s}_{12}^{\Gamma} \equiv\left(s_{12}^{1}, s_{12}^{2}, s_{12}^{3}\right)=\frac{i}{2}\left(\Gamma_{12}^{2} \Gamma_{12}^{3}, \Gamma_{12}^{3} \Gamma_{12}^{1}, \Gamma_{12}^{1} \Gamma_{12}^{2}\right),
\end{gathered}
$$

for $\vec{x}_{\mathrm{D}}$ [1] (333), where the $\Gamma_{12}^{j}$ matrices are given in (324),

$$
\mathrm{s}=(2,1,2,1):
$$

$\vec{s}_{16}^{\Gamma} \equiv\left(s_{16}^{1}, s_{16}^{2}, s_{16}^{3}\right)=\frac{i}{2}\left(\Gamma_{16}^{2} \Gamma_{16}^{3}, \Gamma_{16}^{3} \Gamma_{16}^{1}, \Gamma_{16}^{1} \Gamma_{16}^{2}\right)$,

for $\vec{x}_{\mathrm{D}}[1]$ (363), where the $\Gamma_{16}^{j}$ matrices are given in (353).

It is easy to verify that the generators (82), (83) for any $\mathrm{N}$ commute with the operator of equation (79), and satisfy the commutation relations (21) of the Lie algebra of the Poincare group. The last step in the brief description of the model is the calculation of the Casimir operators $p^{2}=\widehat{p}^{\mu} \widehat{p}_{\mu}, W=w^{\mu} w_{\mu}$ ( $w^{\mu}$ is the Pauli-Lubanski pseudovector) for the fixed value of spin.

\subsection{The example of spin $s=(0,0)$ particle- antiparticle doublet}

The completeness of simplest spin multiplets and doublets consideration of [1] is achieved by the supplementation of this example. The formalism follows from the general formalism of arbitrary spin after the substitution $\mathrm{s}=0$.

The Schrödinger-Foldy equation of RCQM is given by (13) for $\mathrm{N}=1$, i. e. it is 2-component equation. The solution is given by (14) for $\mathrm{N}=1$. The Poincaré group $\mathcal{P}$ generators, with respect to which the equation $(13)$ for $s=(0,0)$ is invariant, are given by (22), (23) taken in the form of $2 \times 2$ matrices with spin terms equal to zero, i. e. the corresponding generators are given by (27)

The corresponding FW type equation of canonical field theory is given by

$$
\left(i \partial_{0}-\sigma^{3} \widehat{\omega}\right) \phi(x)=0
$$

$$
\sigma^{3}=\left|\begin{array}{ll}
1 & 0 \\
0 & -1
\end{array}\right|, \quad \widehat{\omega} \equiv \sqrt{-\Delta+m^{2}} .
$$

The general solution is given by

$\phi(x)=\frac{1}{(2 \pi)^{\frac{3}{2}}} \int d^{3} k\left[e^{-i k x} a^{1}(\vec{k}) \mathrm{d}_{1}+e^{i k x} a^{* 2}(\vec{k}) \mathrm{d}_{2}\right]$.

The Poincaré group $\mathcal{P}$ generators, with respect to which the equation (89) and the set $\{\phi\}$ of its solutions (90) are invariant, have the form

$$
\begin{gathered}
\widehat{p}^{0}=\sigma^{3} \widehat{\omega} \equiv \sigma^{3} \sqrt{-\Delta+m^{2}}, \quad \hat{p}^{\ell}=-i \partial_{\ell}, \\
\hat{j}^{\ell n}=x^{\ell} \widehat{p}^{n}-x^{n} \widehat{p}^{\ell}, \\
\widehat{j}^{0 \ell}=-\hat{j}^{\ell 0}=x^{0} \widehat{p}^{\ell}-\frac{1}{2} \sigma^{3}\left\{x^{\ell}, \widehat{\omega}\right\} .
\end{gathered}
$$

Generators (91), (92) are the partial $2 \times 2$ matrix form of operators (76), (77) taken with the spin terms equal to zero. 


\subsection{The example of covariant field equation for spin $s=(3 / 2,3 / 2)$ particle-antiparticle doublet}

Consider the nontrivial partial example of covariant field equation for arbitrary spin. Such example is given by covariant field equation for spin $s=(3 / 2,3 / 2)$ particle-antiparticle doublet. This case presents the demonstrative example how new equations can be derived by the developed in [1] and here methods.

Now contrary to [1] equation for spin $s=3 / 2$ is found as the simple partial case of general equation (79):

$$
\left[i \partial_{0}-\Gamma_{8}^{0}\left(\vec{\Gamma}_{8} \cdot \vec{p}+m\right)\right] \psi(x)=0 .
$$

Here the $\Gamma_{8}^{\mu}$ matrices are given by

$$
\Gamma_{8}^{0}=\left|\begin{array}{cc}
\mathrm{I}_{4} & 0 \\
0 & -\mathrm{I}_{4}
\end{array}\right|, \quad \Gamma_{8}^{j}=\left|\begin{array}{cc}
0 & \Sigma^{j} \\
-\Sigma^{j} & 0
\end{array}\right|,
$$

where $\Sigma^{j}$ are the $4 \times 4$ Pauli matrices

$$
\Sigma^{j}=\left|\begin{array}{cc}
\sigma^{j} & 0 \\
0 & \sigma^{j}
\end{array}\right|
$$

and $\sigma^{j}$ are the standard $2 \times 2$ Pauli matrices. The matrices $\Sigma^{j}$ satisfy the similar commutation relations as the standard $2 \times 2$ Pauli matrices and have other similar properties. The matrices $\Gamma_{8}^{\mu}$ (89) satisfy the anti-commutation relations of the Clifford-Dirac algebra in the form (29) with $\mathrm{N}=4$.

Note that 8-component equation (93) is not the ordinary direct sum of the two Dirac equations. Therefore, it is not the complex Dirac-Kähler equation [109]. Moreover, it is not the standard 16-component Dirac-Kähler equation [114]. Furthermore, for the same reason it is not the spin $3 / 2$ equation from $[43,45]$. Indeed, the spin $3 / 2$ equation from $[43,45]$ has 12 components. And it is not 16component Rarita-Schwinger equation.

The solution of equation (93) is derived as a partial case from the solution (80) of the general equation (79) and is given by

$$
\begin{gathered}
\psi(x)=V_{8}^{-} \phi(x)= \\
\frac{1}{(2 \pi)^{\frac{3}{2}}} \int d^{3} k\left[e^{-i k x} b^{\mathrm{A}}(\vec{k}) \mathrm{v}_{\mathrm{A}}^{-}(\vec{k})+e^{i k x} b^{* \mathrm{~B}}(\vec{k}) \mathrm{v}_{\mathrm{B}}^{+}(\vec{k})\right],
\end{gathered}
$$

where $\mathrm{A}=\overline{1,4}, \mathrm{~B}=\overline{5,8}$ and the 8 -component spinors $\left(\mathrm{v}_{\mathrm{A}}^{-}(\vec{k}), \mathrm{v}_{\mathrm{B}}^{+}(\vec{k})\right)$ are given by $(257)$ in [1].

The spinors $\left(\mathrm{v}_{\mathrm{A}}^{-}(\vec{k}), \mathrm{v}_{\mathrm{B}}^{+}(\vec{k})\right)$ satisfy the relations of the ortonormalization and completeness similar to the corresponding relations for the standard 4-component Dirac spinors, see, e. g., [113].

In the covariant local field theory, the operators of the SU(2) spin, which satisfy the corresponding commutation relations $\left[s_{8 \mathrm{D}}^{j}, s_{8 \mathrm{D}}^{\ell}\right]=i \varepsilon^{j \ell n} s_{8 \mathrm{D}}^{n}$ and commute with the operator $\left[i \partial_{0}-\Gamma_{8}^{0}\left(\vec{\Gamma}_{8} \cdot \vec{p}+m\right)\right]$ of equation (93), are derived from the pure matrix operators (279) of [1] with the help of transition operator $V_{8}^{-}: \vec{s}_{8 \mathrm{D}}=V_{8}^{-} \vec{s}_{8} V_{8}^{+}$. The explicit form of the transition operator $V_{8}^{\mp}$ is given in (249)-(251) of [1]. The explicit form of these $s=(3 / 2,3 / 2)$ $\mathrm{SU}(2)$ generators was given already by formulas (284) (287) in [1].

The equations on eigenvectors and eigenvalues of the operator $s_{8 \mathrm{D}}^{3}$ (286) in [1] follow from the equations (280) of [1] and the transformation $V_{8}^{-}$. In addition to it, the action of the operator $s_{8 \mathrm{D}}^{3}$ (286) in [1] on the spinors $\left(\mathrm{v}_{\mathrm{A}}^{-}(\vec{k}), \mathrm{v}_{\mathrm{B}}^{+}(\vec{k})\right)(257)$ in [1] also leads to the result

$$
\begin{gathered}
s_{8 \mathrm{D}}^{3} \mathrm{v}_{1}^{-}(\vec{k})= \\
s_{8 \mathrm{D}}^{3} \mathrm{v}_{3}^{-}(\vec{k})=-\frac{3}{2} \mathrm{v}_{1}^{-}(\vec{k}), s_{8 \mathrm{D}}^{3} \mathrm{v}_{2}^{-}(\vec{k})=\frac{1}{2} \mathrm{v}_{2}^{-}(\vec{k}), s_{8 \mathrm{D}}^{3} \mathrm{v}_{4}^{-}(\vec{k})=-\frac{3}{2} \mathrm{v}_{4}^{-}(\vec{k}), \\
s_{8 \mathrm{D}}^{3} \mathrm{v}_{5}^{+}(\vec{k})=\frac{3}{2} \mathrm{v}_{5}^{+}(\vec{k}), \\
s_{8 \mathrm{D}}^{3} \mathrm{v}_{6}^{+}(\vec{k})=\frac{1}{2} \mathrm{v}_{6}^{+}(\vec{k}),
\end{gathered}
$$

$$
s_{8 \mathrm{D}}^{3} \mathrm{v}_{7}^{+}(\vec{k})=-\frac{1}{2} \mathrm{v}_{7}^{+}(\vec{k}), s_{8 \mathrm{D}}^{3} \mathrm{v}_{8}^{+}(\vec{k})=-\frac{3}{2} \mathrm{v}_{8}^{+}(\vec{k}) .
$$

In order to verify equations (97) the identity $(\tilde{\omega}+m)^{2}+$ $(\vec{k})^{2}=2 \tilde{\omega}(\tilde{\omega}+m)$ is used. In the case $\mathrm{v}_{\mathrm{B}}^{+}(\vec{k})$ in the expression $s_{8 \mathrm{D}}^{3}(\vec{k})$ (286) of [1] the substitution $\vec{k} \rightarrow-\vec{k}$ is made.

The equations (97) determine the interpretation of the amplitudes in solution (96). Nevertheless, the direct quantum-mechanical interpretation of the amplitudes should be made in the framework of the RCQM and is already given in [1] (section 14 in paragraph after equations (183)).

The explicit form of the $\mathcal{P}$-generators of the fermionic representation of the Poincaré group $\mathcal{P}$, with respect to which the covariant equation (93) and the set $\{\psi\}$ of its solutions (96) are invariant, is derived as a partial case from the generators (82), (83). The corresponding generators are given by

$$
\begin{gathered}
\widehat{p}^{0}=\Gamma_{8}^{0}\left(\vec{\Gamma}_{8} \cdot \vec{p}+m\right), \quad \widehat{p}^{\ell}=-i \partial_{\ell}, \\
\widehat{j}^{\ell n}=x_{\mathrm{D}}^{\ell} \widehat{p}^{n}-x_{\mathrm{D}}^{n} \widehat{p}^{\ell}+s_{8 \mathrm{D}}^{\ell n} \equiv \widehat{m}^{\ell n}+s_{8 \mathrm{D}}^{\ell n}, \\
\widehat{j}^{0 \ell}=-\widehat{j}^{\ell 0}=x^{0} \widehat{p}^{\ell}-\frac{1}{2}\left\{x_{\mathrm{D}}^{\ell}, \widehat{p}^{0}\right\}+\frac{\widehat{p}^{0}\left(\vec{s}_{8 \mathrm{D}} \times \vec{p}\right)^{\ell}}{\widehat{\omega}(\widehat{\omega}+m)},
\end{gathered}
$$

where the spin matrices $\vec{s}_{8 \mathrm{D}}=\left(s_{8 \mathrm{D}}^{\ell n}\right)$ are given by (284)(286) in [1] and the operator $\vec{x}_{\mathrm{D}}$ has the form

$$
\vec{x}_{\mathrm{D}}=\vec{x}+\frac{i \vec{\Gamma}_{8}}{2 \widehat{\omega}}-\frac{\vec{s}_{8}^{\Gamma} \times \vec{p}}{\widehat{\omega}(\widehat{\omega}+m)}-\frac{i \vec{p}\left(\vec{\Gamma}_{8} \cdot \vec{p}\right)}{2 \widehat{\omega}^{2}(\widehat{\omega}+m)},
$$

with specific spin matrices $\vec{s}_{8}^{\Gamma}$ given in (86).

It is easy to verify that the generators (98), (99) with SU(2) spin (284)-(286) from [1] commute with the operator $\left[i \partial_{0}-\Gamma_{8}^{0}\left(\vec{\Gamma}_{8} \cdot \vec{p}+m\right)\right]$ of equation (93), satisfy 
the commutation relations (21) of the Lie algebra of the Poincaré group and the corresponding Casimir operators are given by $p^{2}=\widehat{p}^{\mu} \widehat{p}_{\mu}=m^{2} \mathrm{I}_{8}, W=w^{\mu} w_{\mu}=$ $m^{2} \vec{s}_{8 \mathrm{D}}^{2}=\frac{3}{2}\left(\frac{3}{2}+1\right) m^{2} \mathrm{I}_{8}$.

The conclusion that equation (93) describes the local field of fermionic particle-antiparticle doublet of the spin $\mathrm{s}=(3 / 2,3 / 2)$ and mass $m>0$ (and its solution (96) is the local fermionic field of the above mentioned spin and nonzero mass) follows from the analysis of equations (97) and the above given calculation of the Casimir operators $p^{2}, W=w^{\mu} w_{\mu}$.

Hence, the equation (93) describes the spin $s=(3 / 2,3 / 2)$ particle-antiparticle doublet on the same level, on which the standard 4-component Dirac equation describes the spin $s=(1 / 2,1 / 2)$ particle-antiparticle doublet. Moreover, the external argument in the validity of such interpretation is the link with the corresponding RCQM of spin $s=(3 / 2,3 / 2)$ particle-antiparticle doublet, where the quantum-mechanical interpretation is direct and evident. Therefore, the fermionic spin $s=(3 / 2,3 / 2)$ properties of equation (93) are proved.

Contrary to the bosonic spin $s=(1,0,1,0)$ properties of the equation (93) found in [1] (section 22), the fermionic spin $\mathrm{s}=(1 / 2,1 / 2,1 / 2,1 / 2)$ properties of this equation are evident. The fact that equation (93) describes the multiplet of two fermions with the spin $s=1 / 2$ and two antifermions with that spin can be proved much more easier then the above given consideration. The proof is similar to that given in the standard 4-component Dirac model. The detailed consideration can be found in sections 7, 9, 10 of [1]. Therefore, equation (93) has more extended property of the Fermi-Bose duality then the standard Dirac equation [85-91]. This equation has the property of the Fermi-Bose triality. The property of the Fermi-Bose triality of the manifestly covariant equation (93) means that this equation describes on equal level (i) the spin $s=(1 / 2,1 / 2,1 / 2,1 / 2)$ multiplet of two spin $s=(1 / 2,1 / 2)$ fermions and two spin $s=(1 / 2,1 / 2)$ antifermions, (ii) the spin $s=(1,0,1,0)$ multiplet of the vector and scalar bosons together with their antiparticles, (iii) the spin $\mathrm{s}=(3 / 2,3 / 2)$ particle-antiparticle doublet.

It is evident that equation (93) is new in comparison with the Pauli-Fierz [41], Rarita-Schwinger [57] and confirmed by Davydov [58] equations for the spin $s=3 / 2$ particle. Contrary to 16 -component equations from $[41,57,58]$ equation (93) is 8-component and does not need any additional condition. Only formally equation (93) looks like to have some similar features with the Bargman-Wigner equation [43] for arbitrary spin, when the spin value is taken $3 / 2$. The transformation $V_{8}^{\mp}=\frac{\mp \vec{\Gamma}_{8} \cdot \vec{p}+\widehat{\omega}+m}{\sqrt{2 \widehat{\omega}(\widehat{\omega}+m)}}$ looks like the transformation of Pursey [45] in the case of $s=3 / 2$. Nevertheless, the difference is clear. Equation (93) is not the ordinary direct sum of the two Dirac equations. Furthermore, the given here model is derived from the first principles of RCQM (not from the FW type representation of the canonical field theory). Our consideration is original and new. The link with corresponding RCQM, the proof of the sym- metry properties and relativistic invariance, the well defined spin operator (284)-(286) in [1], the features of the FermiBose duality (triality) of the equation (93), the interaction with electromagnetic field and many other characteristics are suggested firstly. Finally, the Bargman-Wigner equation [43] in the case $s=3 / 2$ has 12 components. The comparison with Bhabha equation is evident as well. Bhabha have proved itself [78] that his equation in the case $s=3 / 2$ coincide with Rarita-Schwinger equation, i.e. has 16 components.

Interaction, quantization and Lagrange approach in the above given spin $s=(3 / 2,3 / 2)$ model are completely similar to the Dirac 4-component theory and standard quantum electrodynamics. For example, the Lagrange function of the system of interacting 8-component spinor and electromagnetic fields (in the terms of 4-vector potential $A^{\mu}(x)$ ) is given by

$$
\begin{gathered}
L=-\frac{1}{4} F^{\mu \nu} F_{\mu \nu}+\frac{i}{2}\left(\bar{\psi}(x) \Gamma_{8}^{\mu} \frac{\partial \psi(x)}{\partial x^{\mu}}-\frac{\partial \bar{\psi}(x)}{\partial x^{\mu}} \Gamma_{8}^{\mu} \psi(x)\right)- \\
m \bar{\psi}(x) \psi(x)+q \bar{\psi}(x) \Gamma_{8}^{\mu} \psi(x) A_{\mu}(x)
\end{gathered}
$$

where $\bar{\psi}(x)$ is the independent Lagrange variable and $\bar{\psi}=$ $\psi^{\dagger} \Gamma_{8}^{0}$ in the space of solutions $\{\psi\}$. In Lagrangian (101) $F_{\mu \nu}=\partial_{\mu} A_{\nu}-\partial_{\nu} A_{\mu}$ is the electromagnetic field tensor in the terms of potentials, which play the role of variational variables in this Lagrange approach.

Therefore, the covariant local quantum field theory model for the interacting particles with spin $s=3 / 2$ and photons can be constructed in complete analogy to the construction of the modern quantum electrodynamics. This model can be useful for the investigations of processes with interacting hyperons and photons.

\subsection{The example of covariant field equation for spin $s=(2,2)$ particle-antiparticle dou- blet}

The canonical Foldy-Wouthuysen type field equation and its solution are given by

$$
\begin{gathered}
\left(i \partial_{0}-\Gamma_{10}^{0} \widehat{\omega}\right) \phi(x)=0 \\
\Gamma_{10}^{0}=\left|\begin{array}{ll}
\mathrm{I}_{5} & 0 \\
0 & -\mathrm{I}_{5}
\end{array}\right|, \quad \widehat{\omega} \equiv \sqrt{-\Delta+m^{2}} \\
\phi(x)=\frac{1}{(2 \pi)^{\frac{3}{2}}} \int d^{3} k\left[e^{-i k x} g^{\mathrm{A}}(\vec{k}) \mathrm{d}_{\mathrm{A}}+e^{i k x} g^{* \mathrm{~B}}(\vec{k}) \mathrm{d}_{\mathrm{B}}\right],
\end{gathered}
$$

$\mathrm{A}=1,2,3,4,5, \mathrm{~B}=6,7,8,9,10$, here $\{\mathrm{d}\}$ are the 10 component Cartesian orts.

The only small difficulty is as follows. The covariant field theory equation for the spin $s=(2,2)$ particleantiparticle doublet can not be presented in terms of $\Gamma$ matrices. Indeed, the $10 \times 10$ Clifford-Dirac $\Gamma$ matrices are not exist as an mathematical objects. Therefore, one need to look for the locally covariant field equations for spin 
$s=(2,2)$ particle-antiparticle doublet not in the terms of $\Gamma$ matrices.

Consider two ways of the derivation of the covariant field equations for spin $s=(2,2)$ particle-antiparticle doublet. The first way is based on the application of the corresponding Foldy-Wouthuysen transformation directly to the canonical field equation (102). Note that there is a nonzero chance to find the appropriate transformation among a big number of so-called Foldy-Wouthuysen transformations for an arbitrary spin [115-124]. Unfortunately for our approach, many authors considered for the spin $s=1$ case the 4-vector potential (see, e. g., [124]) and not 3-component wave function $\vec{\psi}=\vec{E}-i \vec{E}$ as it is in $[1-3,11,12,84,89]$. Therefore, the objects for spin $\mathrm{s}=2$ case are different as well. Indeed, our approach for arbitrary spin equations differs from other known approaches.

Hence, below (see some details in [125]) another way of the derivation of the covariant field equations for spin $s=(2,2)$ particle-antiparticle doublet is realized. It is easy to construct the formalism of the relativistic canonical quantum mechanics of the spin $\mathrm{s}=(2,0,2,0)$ particle-antiparticle multiplet. Further, the canonical Foldy-Wouthuysen type model of the spin $s=(2,0,2,0)$ particle-antiparticle field can be formulated. Moreover, both models are presented already in sections 16 and 26 of [1]. After that the $12 \times 12$ operator transformation (78) should be applied for the canonical Foldy-Wouthuysen type equation of the spin $\mathrm{s}=(2,0,2,0)$ particle-antiparticle multiplet.

Found by the above considered way (or found as the partial $12 \times 12$ case of arbitrary spin equation (79)) covariant field equation for the spin $s=(2,0,2,0)$ particle-antiparticle multiplet is given by

$$
\begin{aligned}
& {\left[i \partial_{0}-\Gamma_{12}^{0}\left(\vec{\Gamma}_{12} \cdot \vec{p}+m\right)\right] \psi(x)=0,} \\
& \Gamma_{12}^{0}=\left|\begin{array}{cc}
\mathrm{I}_{6} & 0 \\
0 & -\mathrm{I}_{6}
\end{array}\right|, \quad \Gamma_{12}^{j}=\left|\begin{array}{cc}
0 & \Sigma_{6}^{j} \\
-\Sigma_{6}^{j} & 0
\end{array}\right|,
\end{aligned}
$$

where $\Sigma_{6}^{j}$ are the $6 \times 6$ Pauli matrices $\Sigma_{6}^{1}=$ $\left|\begin{array}{cc}0 & \mathrm{I}_{3} \\ \mathrm{I}_{3} & 0\end{array}\right|, \Sigma_{6}^{2}=\left|\begin{array}{cc}0 & -i \mathrm{I}_{3} \\ i \mathrm{I}_{3} & 0\end{array}\right|, \Sigma_{6}^{3}=\left|\begin{array}{cc}\mathrm{I}_{3} & 0 \\ 0 & -\mathrm{I}_{3}\end{array}\right|$. The general solution of the equation (34) is given by the formula (327) in [1].

In order to find the covariant field equations for spin $\mathrm{s}=(2,2)$ particle-antiparticle doublet one can put equal to zero the scalar components in (104). The place of the scalar components is evident from the corresponding quantummechanical consideration (section 16 of [1]).

The similar way was demonstrated in [1] (section 23) to find the covariant Maxwell-like field equations for the spin $\mathrm{s}=(1,1)$ particle-antiparticle doublet from the known Diraclike equation (252) in [1] for the spin $s=(1,0,1,0)$ particleantiparticle multiplet. Similarly to equation (275) in [1] the covariant field equation (105) (or (106)), written below for the spin $\mathrm{s}=(2,2)$ particle-antiparticle doublet, can be presented in terms of curl, div and grad operators as well.
Thus, the locally covariant field equations for 10 component spin $\mathrm{s}=(2,2)$ particle-antiparticle doublet found by this way has the form

$$
\begin{gathered}
i \partial_{0} \psi^{1}-p^{3} \psi^{6}-m \psi^{1}=0, \\
i \partial_{0} \psi^{2}-p^{3} \psi^{7}+i p^{2} \psi^{10}-p^{1} \psi^{10}-m \psi^{2}=0, \\
i \partial_{0} \psi^{3}-p^{3} \psi^{8}+i p^{2} \psi^{9}-p^{1} \psi^{9}-m \psi^{3}=0 \\
i \partial_{0} \psi^{4}+p^{3} \psi^{9}-i p^{2} \psi^{8}-p^{1} \psi^{8}-m \psi^{4}=0, \\
i \partial_{0} \psi^{5}+p^{3} \psi^{10}-i p^{2} \psi^{7}-p^{1} \psi^{7}-m \psi^{5}=0, \\
i \partial_{0} \psi^{6}-p^{3} \psi^{1}+m \psi^{6}=0, \\
i \partial_{0} \psi^{7}-p^{3} \psi^{2}+i p^{2} \psi^{5}-p^{1} \psi^{5}+m \psi^{7}=0, \\
i \partial_{0} \psi^{8}-p^{3} \psi^{3}+i p^{2} \psi^{4}-p^{1} \psi^{4}+m \psi^{8}=0, \\
i \partial_{0} \psi^{9}+p^{3} \psi^{4}-i p^{2} \psi^{3}-p^{1} \psi^{3}+m \psi^{9}=0, \\
i \partial_{0} \psi^{10}+p^{3} \psi^{5}-i p^{2} \psi^{2}-p^{1} \psi^{2}+m \psi^{10}=0, \\
-i p^{2} \psi^{1}-p^{1} \psi^{1}=0, \quad-i p^{2} \psi^{6}-p^{1} \psi^{6}=0,
\end{gathered}
$$

or the Dirac-like form

$$
\begin{gathered}
{\left[i \partial_{0}-\Gamma_{8}^{0}\left(\vec{\Gamma}_{8} \cdot \vec{p}+m\right)\right] \psi(x)=0,} \\
\left(i \partial_{0}-\sigma^{1} p^{3}-\sigma^{3} m\right) \chi=0, \quad\left(p^{1}+i p^{2}\right) \chi=0,
\end{gathered}
$$

where $\psi=\operatorname{column}\left(\psi^{1}, \psi^{2}, \ldots, \psi^{8}\right), \chi=\left|\begin{array}{c}\psi^{9} \\ \psi^{10}\end{array}\right|, \Gamma_{8}^{0}=$ $\left|\begin{array}{cc}\mathrm{I}_{4} & 0 \\ 0 & -\mathrm{I}_{4}\end{array}\right|, \Gamma_{8}^{j}=\left|\begin{array}{cc}0 & \Sigma_{4}^{j} \\ -\Sigma_{4}^{j} & 0\end{array}\right|$, and $\Sigma_{4}^{j}$ are the $4 \times 4$ Pauli matrices $\Sigma_{4}^{1}=\left|\begin{array}{cc}0 & \mathrm{I}_{2} \\ \mathrm{I}_{2} & 0\end{array}\right|, \Sigma_{4}^{2}=$ $\begin{array}{lc}0 & -i \mathrm{I}_{2} \\ i \mathrm{I}_{2} & 0\end{array}\left|, \Sigma_{4}^{3}=\right| \begin{array}{cc}\mathrm{I}_{2} & 0 \\ 0 & -\mathrm{I}_{2}\end{array} \mid$. The form similar to (275) of [1] is found by the method similar to one presented in section 23 of [1].

\section{Briefly on the different ways of the Dirac equation derivation}

Among the results of this paper the original method of the derivation of the standard Dirac and the Dirac-like equation for arbitrary spin is suggested. The Dirac equation is derived here from the 4-component formalism of RCQM. In order to determine the place of this derivation among the other known methods consider below the different ways of the Dirac equation derivation.

One should note the elegant derivation given by Paul Dirac in his book [5]. Until today it is very interesting for the readers to feel Dirac's way of thinking and to follow his logical steps. Nevertheless, the Dirac's consideration of the Schrödinger-Foldy equation, which was essentially used in his derivation [5], was not correct. Especially his assertion that Schrödinger-Foldy equation is unsatisfactory from the point of view of the relativistic theory. Dirac's point of view is considered here in section 2. Dirac's doubts 
were overcome in $[6,7,14]$. Today the significance of the Schrödinger-Foldy equation is confirmed by more then a hundred publications about FW (see, e. g., [31, 45, 50, 115-128] and the references therein) and the spinless Salpeter equations [8, 13, 20-30, 32, 33, 35, 36], which have wide-range application in contemporary theoretical physics.

In the well-known book [113] one can find a review of the Dirac theory and two different ways of the Dirac equation derivation. First, it is the presentation of the KleinGordon equation in the form of a first-order differential system of equations, factorization of the Klein-Gordon operator. Second, the Lagrange approach is considered and the Dirac equation is derived from the variational EulerLagrange least action principle.

In van der Waerden-Sakurai derivation [129] of the Dirac equation the spin of the electron is incorporated into the nonrelativistic theory. The representation of the nonrelativistic kinetic energy operator of the free spin $1 / 2$ particle in the form $H^{\mathrm{KE}}=(\vec{\sigma} \cdot \vec{p})(\vec{\sigma} \cdot \vec{p}) / 2 m$ and the relativistic expression $E^{2}-\vec{p}^{2}=m^{2}$ are used. Then the procedure of transition from 2-component to 4-component equation is fulfilled and explained.

In the book [130] (second edition) the Dirac equation is derived from the manifestly covariant transformational properties of the 4-component spinor.

The derivation of the Dirac equation from the initial geometric properties of the space-time and electron together with wide-range discussion of the geometric principles of the electron theory is the main content of the book [131]. The ideas of V. Fock and D. Iwanenko [132, 133] on the geometrical sense of Dirac $\gamma$-matrices are the basis of the approach.

One should point out the derivation of the Dirac equation based on the Bargman-Wigner classification of the irreducible unitary representations of the Poincaré group, see e. g. [134]. It is an illustrative demonstration of the possibilities of the group-theoretical approach to the elementary particle physics.

Dirac equation is the partial case of the Bargman-Wigner equation [43]. Indeed, after the substitution $s=1 / 2$ into $2 \mathrm{~s} \cdot 4$ component Bargman-Wigner equation [43] one comes to 4-component Dirac equation. Nevertheless, such derivation is week. Note that Bargman-Wigner equation has been introduced as the 2s.4-component generalization of the Dirac equation.

In L. Foldy's papers [6, 7, 14] one can easy find the inverse problem, in which the Dirac equation is obtained from the FW equation. Nevertheless, it is only the transition from one representation of the spinor field to another.

H. Sallhofer [135, 136] derived the Dirac equation for hydrogen spectrum starting from the Maxwell equations in medium. Strictly speaking, only the stationary equations were considered. He named his derivation "The MaxwellDirac isomorphism". In the paper [137] the demonstrative variant was suggested. The complete set of corresponding transformation was considered. If to speak carefully, it is not another derivation as it was mentioned recently in [138]. Note that "isomorphism" in [135-137] can be considered rather than the physical not as the mathematical assertion. In the papers [139-145] we named it (together with the case of free Maxwell equations in vacuum) as the "relationship" between the Dirac and the Maxwell equations. J. Keller in his review [146] named it the "mapping" of the Maxwell formalism into the Dirac formalism.

In [147] the derivation of [130] was repeated together with taking into account the physical meaning of the negative energies and the relative intrinsic parity of the elementary particles. The authors believe that the derivation from [130] is improved.

In [148] quaternion measurable processes were introduced and the Dirac equation was derived from the Langevin equation associated with a two-valued process.

The author of [149] was able to derive the Dirac equation from the conservation law of spin $1 / 2$ current. The requirement that this current is conserved leads to a unique determination of the Lorentz invariant equation satisfied by the relativistic spin $1 / 2$ field. Let us briefly comment that the complete list of conservation laws for the Dirac theory is the Noether consequence of the Dirac equation. Therefore, the validity of the inverse problem is really expected. Can it be considered an independent derivation?

The Dirac equation was derived [150] from the master equation of Poisson process by analytic continuation. The extension to the case where a particle moves in an external field was given. It was shown that the generalized master equation is closely related to the three-dimensional Dirac equation in an external field.

In [151], a method of deriving the Dirac equation from the relativistic Newton's second law was suggested. Such derivation is possible in a new formalism, which relates the special form of relativistic mechanics to the quantum mechanics. The author suggested a concept of a velocity field. At first, the relativistic Newton's second law was rewritten as a field equation in terms of the velocity field, which directly reveals a new relationship linked to the quantum mechanics. After that it was shown that the Dirac equation can be derived from the field equation in a rigorous and consistent manner.

A geometrical derivation of the Dirac equation, by considering a spin $1 / 2$ particle traveling with the speed of light in a cubic space-time lattice, was made in [152]. The mass of the particle acts to flip the multi-component wave function at the lattice sites. Starting with a difference equation for the case of one spatial and one time dimensions, the authors generalize the approach to higher dimensions. Interactions with external electromagnetic and gravitational fields are also considered. Nevertheless, the idea of such derivation is based on the Dirac's observation that the instantaneous velocity operators of the spin $1 / 2$ particle (hereafter called by the generic name $i$ the electron $i$ i) have eigenvalues $\pm c$. This mistake of Dirac was demonstrated and overcome in Ref. [6].

Using the mathematical tool of Hamilton's bi- 
quaternions, the authors of [153] propose a derivation of the Dirac equation from the geodesic equation. Such derivation is given in the program of application of the theory of scale relativity to the purposes of microphysics at recovering quantum mechanics as a new non-classical mechanics on a non-derivable space-time.

M. Evans was successful to express his equation of general relativity (generally covariant field equation for gravitation and electromagnetism [154]) in spinor form, thus producing the Dirac equation in general relativity [155]. The Dirac equation in special relativity is recovered in the limit of Euclidean or flat space-time.

Author of [156] first determines that each eigenfunction of a bound particle is a specific superposition of plane wave states that fulfills the averaged energy relation. After that the Schrodinger and Dirac equations were derived as the unique conditions the wave function must satisfy at each point in order to fulfill the corresponding energy equation. The Dirac equation involving electromagnetic potentials has been derived.

In [157] the Dirac equation is derived by conformal differential geometry. The Hamilton-Jacobi equation for the particle is found to be linearized, exactly and in closed form, by an ansatz solution that can be straightforwardly interpreted as the quantum wave function of the 4-spinor solution of Diracs equation. All quantum features arise from the subtle interplay between the conformal curvature acting on the particle as a potential and the particle motion which affects the geometric prepotential associated to the conformal curvature itself. Finally, the Dirac equation is found in the form $\widehat{D}_{+} \widehat{D}_{-} \psi=\widehat{D}_{-} \widehat{D}_{+} \psi=0, \widehat{D}_{\mp} \equiv$ $\gamma^{\mu}\left(p_{\mu}-e A_{\mu}\right) \mp m$.

Recently, in [158] the derivation of the Dirac equation from principles of information processing has been presented. It has been shown, without using the relativity principle, how the Dirac equation in three space-dimensions emerges from the large-scale dynamics of the minimal nontrivial quantum cellular automaton satisfying unitarity, locality, homogeneity, and discrete isotropy. The Dirac equation is recovered for small wave-vector and inertial mass, whereas Lorentz covariance is distorted in the ultrarelativistic limit. The automaton can thus be regarded as a theory unifying scales from Planck to Fermi.

More then ten years ago we already presented our own derivation of the Dirac equation [142-145]. The Dirac equation was derived from slightly generalized Maxwell equations with gradient-like current and charge densities. This form of the Maxwell equations, which is directly linked with the Dirac equation, is the maximally symmetrical variant of these equations. Such Maxwell equations are invariant with respect to a 256-dimensional algebra (the wellknown algebra of conformal group has only 15 generators). Of course, we derived only massless Dirac equation.

In our recent papers [11, 12], and in [1-3], the Dirac equation has been derived from the 4-component Schrödinger-Foldy equation (13) of the RCQM. The starting point RCQM model of the spin $s=(1 / 2,1 / 2)$ particle- antiparticle doublet has been formulated in these articles as well. Hence, the Dirac equation has been derived from the more fundamental model of the same physical reality, which is presented by the RCQM of the spin $s=(1 / 2,1 / 2)$ particle-antiparticle doublet.

Thus, a review of the different derivations of the Dirac equation demonstrates that presented in [1-3] and here method of the Dirac equation (and the Dirac-like equation for arbitrary spin) derivation is original and new.

It follows from the above given consideration that new methods of the Dirac equation derivation are not stopped and to be continued.

\section{Interaction}

This paper in general is about free non-interacting fields and particle states. Note at first that the free non-interacting fields and particle states are the physical reality of the same level as the interacting fields and corresponding particle states. Nevertheless, it is meant that the interaction between a fields can be easily introduced on the every step of consideration. One test model with interaction is considered in explicit form in the section 7 above. The interaction can not be the deficiency in these constructions and can be introduced in many places by the method similar to the formula (101).

Note at first that the free non-interacting fields and particle states are the physical reality of the same level as the interacting fields and corresponding particle states. The best well-known example is the free Maxwell electromagnetic field in the form of free electromagnetic waves. Therefore, the systematic investigation of free non-interacting fields and particle states on the different levels of the relativistic canonical quantum mechanics of arbitrary spin (based on the Schrodinger-Foldy equation), the relativistic canonical field theory of arbitrary spin (based on the FW equation and its generalizations), the locally covariant field theory of arbitrary spin (based on the Dirac equation and its generalizations) and, moreover, the introduction of the operator link between these theories is some result as well and, I hope, have some independent significance. The precise free field consideration is the necessary first step in all consequent field theory presentation, in axiomatic presentation as well. Further, it is well-known that when the free field models are well-defined the interaction between them can be introduced very easy in well-defined forms known from the literature.

The free particle RCQM today is the subject of other authors considerations as well. Note that the wave packet solutions of the Salpeter equation and their unusual properties (rather recent papers $[8,34]$ ) have been derived for the free non-interacting cases.

Moreover, for the standard Dirac theory the interaction is given in all handbooks on the relativistic quantum field theory and quantum electrodynamics. Such consideration can be added here very easy. Nevertheless, here one typi- 
cal interaction is considered already as the test example in order to demonstrate the way of introduction of the interaction in corresponding models. For the spin $s=(3 / 2,3 / 2)$ field the interaction with electromagnetic potentials was given in the section 7 in the formula (101). Therefore, I had a thought that it is enough to explain the situation with interaction here. The interaction can not be the deficiency in these constructions and can be introduced in many places by the method similar to the formula (101).

Other concrete physical effects and the problem of description of (inter)-particle interactions in frames of the RCQM are described by other authors, see the references [24-36].

\section{Application to the discussion around the antiparticle negative mass}

The system of vertical and horizontal links between the RCQM and the field theory, which is proved above, has different useful applications. One of the fundamental applications is the participation in the discussion around the antiparticle negative mass. We emphasize that the model of the RCQM and the corresponding field theory do not need the appealing to the antiparticle negative mass concept [159-162]. It is natural due to the following reasons.

It is only the energy which depends on the mass. And the total energy together with the momentum is related to the external degrees of freedom, which are common and the same for the particle and antiparticle (for the electron and positron). The difference between the particle and the antiparticle consists only in internal degrees of freedom such as the spin $\vec{s}$ and the sign of the charge $g=-\gamma^{0}$. Thus, if in the RCQM the mass of the particle is taken positive then the mass of the antiparticle must be taken positive too.

On the other hand, a comprehensive analysis [160] of the Dirac equation for the doublet had led the authors of [160] to the concept of the negative mass of the antiparticle. Therefore, the consideration here gives the additional arguments that the Dirac model (or the Foldy-Wouthuysen model related to it) is not the quantum-mechanical one. Furthermore, in the problem of the relativistic hydrogen atom the use of the negative-frequency part $\psi^{-}(x)=$ $e^{-i \omega t} \psi(\vec{x})$ of the spinor $\psi(x)$ in the "role of the quantummechanical object" is not valid. In this case neither $|\psi(\vec{x})|^{2}$, nor $\bar{\psi}(\vec{x}) \psi(\vec{x})$ is the probability distribution density with respect to the eigenvalues of the Fermi-doublet coordinate operator. It is due to the fact [6] that in the Dirac model the $\vec{x}$ is not the experimentally observable Fermidoublet coordinate operator.

The application of the RCQM can be useful for the analysis of the experimental situation found in [163]. Such analysis is interesting due to the fact that (as it is demonstrated here and in [1-3]) the RCQM is the most fundamental model of the particle-antiparticle doublet.
Another interesting application of the RCQM is inspired by [164], where the quantum electrodynamics is reformulated in the FW representation. The author of [164] essentially used the result of [160] on the negative mass of the antiparticle. Starting from the RCQM we are able not to appeal to the concept of the antiparticle negative mass.

\section{On the interpretation of $\mathrm{Cl}^{\mathrm{R}}(\mathbf{0 , 6})$ and $\mathrm{SO}(8)$ algebras}

The gamma matrix representation of 64-dimensional $\mathrm{Cl}^{\mathrm{R}}(0,6)$ algebra was put into consideration in [85-91]. The algebra was considered over the field of real numbers and for the $4 \times 4 \gamma$ matrices. The algebra is described above in subsection 6.8. Mathematically correct interpretation of $\mathrm{Cl}^{\mathrm{R}}(0,6)$ algebra was given in $[2,3,125,165]$.

The gamma matrix representation of $\mathrm{SO}(8)$ algebra containing two independent sets of SU(2) generators was put into consideration in [88-91]. The algebra was considered over the field of real numbers, in terms of anti-Hermitian operators and for the $4 \times 4 \gamma$ matrices. The 28 generators of this algebra are given above in (45). For the partial case of $4 \times 4$ matrices one comes if to put $\mathrm{N}=2$ in (45). The commutation relation are given by (46). The interesting problem known from [89-91] is the relationship between the 64-dimensional $\mathrm{Cl}^{\mathrm{R}}(0,6)$ and 28-dimensional $\mathrm{SO}(8)$ algebras.

Recently in [166] "the exclusive representations of the extended (29-dimensional) real-number Clifford-Dirac algebra are constructed for the spinor field." Nevertheless, even this first sentence of the abstract of [166] contains many errors. (i) The Clifford algebra (and Clifford-Dirac matrix representation as well) can not be 29-dimensional because the dimension of $\mathrm{Cl}^{\mathrm{R}}(\mathrm{m}, \mathrm{n})$ algebra is $2^{m+n}$. Therefore, the chain of possible dimensions is given by 16,32 , $64,128, \ldots$ (ii) For the same reasons the 29-dimensional algebra can not be any subalgebra of the Clifford algebra. (iii) Further, such representations can not be "exclusive" because all gamma matrix representations of that kind were considered already in [88-91] and [2, 3, 125, 165]. (iiii) Furthermore, 29 dimensions are related in [166] with $\mathrm{SO}(8)$ algebra. Unfortunately, the algebra $\mathrm{SO}(8)$ belongs to Lie algebras and Lie algebras does not contain unit element. Therefore, the dimension of $\mathrm{SO}(8)$ algebra is 28 , not $28+1$. (V) It is evident that any Lie algebra can not coincide (or be the subalgebra) of any Clifford algebra. Clifford and Lie algebras are different algebras with different structure. For example, contrary to Lie algebras, Clifford algebras contain the unit element. (VI) The chain of possible dimensions for $\mathrm{SO}(\mathrm{m} . \mathrm{n})$ or $\mathrm{SO}(\mathrm{n})$ algebras is given by $3,6,10,15,21,28$, $36,45,55,66, \ldots$ Therefore, the most close to each other are 16-dimensional Clifford $\mathrm{Cl}^{\mathrm{C}}(1,3)$ and 15-dimensional $\mathrm{SO}(1,5)$ algebras over the field of complex numbers. Below in Table 1 and Table 2 the explicit forms of corresponding gamma matrix representations are compared. The 
difference between the elements of these representations is evident. The comparison of introduced in [88-91] gamma matrix representations of $\mathrm{Cl}^{\mathrm{R}}(0,6)$ and $\mathrm{SO}(8)$ algebras is given in $[110,167]$.

The errors related above to the first sentence of the abstract of [166] are repeated during all sections of [166]. Therefore, the well-defined mathematical analysis given in $[110,167]$ and here is necessary for further applications. The analysis of physical phenomena is impossible without mathematical correctness of consideration. Hence, we must improve the paper [166].

Table 1. 15 elements of the $\gamma$ matrix representation of the $\mathrm{SO}(1,5)$ algebra

\begin{tabular}{|c|c|c|c|c|}
\hline$\frac{1}{2} \gamma^{0} \gamma^{1}$ & $\frac{1}{2} \gamma^{0} \gamma^{2}$ & $\frac{1}{2} \gamma^{0} \gamma^{3}$ & $\frac{1}{2} \gamma^{0} \gamma^{4}$ & $\frac{1}{2} \gamma^{0} \gamma^{5} \equiv \frac{1}{2} \gamma^{0}$ \\
\hline & $\frac{1}{2} \gamma^{1} \gamma^{2}$ & $\frac{1}{2} \gamma^{1} \gamma^{3}$ & $\frac{1}{2} \gamma^{1} \gamma^{4}$ & $\frac{1}{2} \gamma^{1} \gamma^{5} \equiv \frac{1}{2} \gamma^{1}$ \\
\hline & & $\frac{1}{2} \gamma^{2} \gamma^{3}$ & $\frac{1}{2} \gamma^{2} \gamma^{4}$ & $\frac{1}{2} \gamma^{2} \gamma^{5} \equiv \frac{1}{2} \gamma^{2}$ \\
\hline & & & $\frac{1}{2} \gamma^{3} \gamma^{4}$ & $\frac{1}{2} \gamma^{3} \gamma^{5} \equiv \frac{1}{2} \gamma^{3}$ \\
\hline & & & & $\frac{1}{2} \gamma^{4} \gamma^{5} \equiv \frac{1}{2} \gamma^{4}$ \\
\hline
\end{tabular}

Table 2. 16 elements of the $\gamma$ matrix representation of the $\mathrm{Cl}^{\mathrm{C}}(1,3)$ algebra ( $\mathrm{I}_{4}=-\gamma^{0} \gamma^{1} \gamma^{2} \gamma^{3} \gamma^{4}$ is $4 \times 4$ unit matrix)

\begin{tabular}{|l|l|l|l|l|l|}
\hline$I_{4}$ & & & & & \\
\hline & $\gamma^{0} \gamma^{1}$ & $\gamma^{0} \gamma^{2}$ & $\gamma^{0} \gamma^{3}$ & $\gamma^{0} \gamma^{4}$ & $\gamma^{0} \gamma^{5} \equiv \gamma^{0}$ \\
\hline & & $\gamma^{1} \gamma^{2}$ & $\gamma^{1} \gamma^{3}$ & $\gamma^{1} \gamma^{4}$ & $\gamma^{1} \gamma^{5} \equiv \gamma^{1}$ \\
\hline & & & $\gamma^{2} \gamma^{3}$ & $\gamma^{2} \gamma^{4}$ & $\gamma^{2} \gamma^{5} \equiv \gamma^{2}$ \\
\hline & & & & $\gamma^{3} \gamma^{4}$ & $\gamma^{3} \gamma^{5} \equiv \gamma^{3}$ \\
\hline & & & & & $\gamma^{4} \gamma^{5} \equiv \gamma^{4}$ \\
\hline
\end{tabular}

\section{On the possible relation to SUSY}

(i) SUSY partner for considered in [85-91] and in [166] spin $1 / 2$ particle-antiparticle doublet $s=(1 / 2,1 / 2)$ is the spin 0 particle-antiparticle doublet $s=(0,0)$. In some variants of SUSY the role of such super-partner can play the spin 1 particle-antiparticle doublet $s=(1,1)$. In [85-91] and in [166] another object, namely spin $s=(1,0)$ particleantiparticle multiplet, has been considered. Thus, results of [85-91] and [166] do not relate to SUSY.

(ii) SUSY is based on fermion-boson super-multiplets. Such objects are not considered in [85-91] and in [166]. Thus, results of [85-91] and [166] do not relate to SUSY.

(iii) SUSY is based on super-space, which in addition to Minkowski space-time contains non-commutative spinor coordinates. Such objects are not considered in [85-91] and in [166] as well. Thus, results of [85-91] and [166] do not relate to SUSY as well.

Moreover, super-partners with essentially large mass in comparison with ordinary elementary particles are absent in [85-91] and in [166].

It means that above mentioned concepts, definitions and notations determined SUSY as the concrete fixed model of physical reality. It is evident that in [85-91] and in [166] we deal with another theoretical model. In [85-91] following [81] such phenomena were named the Fermi-Bose duality.

The authors of [166] named their article "Extension of the standard CD algebra in the axiomatic approach for spinor field and Fermi-Bose duality". Fermi-Bose duality is good title. Nevertheless, in subsection 3.4 of [166] analysis of SUSY partner for $e^{-} e^{+}$-doublet is presented. The brief analysis given above demonstrates that the consideration of SUSY in subsection 3.4 of [166] is senseless. If in future we will be able to construct on the basis of [85-91] the new principal model of physical reality it will be not contemporary SUSY.

Another question arises "Why somebody needs to follow SUSY today?" Note that today hypothesis on SUSY is not so popular as 20 years ago!

\section{Discussions and conclusions}

In the presented above text (and briefly in [2, 3]) our experience $[11,12,84-91,107-110,137,139-145]$ in the time span 2002-2013 in the investigation of the spin $s=1 / 2$ and $s=1$ fields is applied for the first time to the higher spin cases $s=3 / 2$ and $s=2$ (in the form of electronic preprint it has been fulfilled for the first time in [1]). Thus, our "old" papers are augmented by the list of new results for higher spins and generalization for arbitrary spin. Moreover, here (the start has been given in [1]) the system of different vertical and horizontal links between the arbitrary spin particles descriptions on the levels of relativistic quantum mechanics, canonical field theory (of FW type) and locally covariant field theory is suggested.

Among the results of this paper the original method of derivation of the Dirac (and the Dirac-like equations for higher spins) is suggested (section 7). In order to determine the place of this derivation among the other known methods in section 8 the different ways of the Dirac equation derivation [5, 129-137, 139-145, 147-158] have been reviewed. Thus, a review of the different derivations of the Dirac equation demonstrates that presented in section 7 general method is original and new. Here the Dirac equation is derived from the Schrödinger-Foldy equation (13) (in 4component case) of the RCQM. The RCQM model of the spin $s=(1 / 2,1 / 2)$ particle-antiparticle doublet has been considered in details in section 7 of [1]. Hence, the Dirac equation is derived in [1-3] and here from the more fundamental model of the same physical reality, which is presented by the RCQM of the spin $s=(1 / 2,1 / 2)$ particle-antiparticle doublet. 
One of the general fundamental conclusions is as follows. It is shown by corresponding comparison that customary FW representation can not give the complete quantum-mechanical description of the relativistic particle (or particle multiplet). Compare, e. g. the equations on eigenvectors and eigenvalues for the third component of the spin operator in each quantum-mechanical and canonical field theory model here above and in [1-3]. It is useful also to compare the general solutions in RCQM and in field theory (it is enough to consider the field theory in FW representation). Contrary to RCQM in FW representation the general solution consists of positive and negative frequency parts. As a consequence contrary to RCQM in FW representation the energy has an indefinite sign. Hence, the complete quantum-mechanical description of the relativistic particle (or particle multiplet) can be given only in the framework of the RCQM. Therefore, the customary FW transformation is extended here to the form, which gives the link between the locally covariant field theory and the RCQM. Hence, such extended inverse FW transformation is used here to fulfill the synthesis of covariant particle equations. The start of such synthesis is given here from the RCQM and not from the canonical field theory in the representations of the Foldy-Wouthuysen type.

Comparison of RCQM and FW representation visualizes the role of the J. von Neumann axiomatic [38] in this presentation. Therefore, relation of J. von Neumann axiomatic [38] to the overall contents of the paper is direct and unambiguous. It is shown that among the above considered models only RCQM of arbitrary spin can be formulated in J. von Neumann's axiomatic, whereas canonical and covariant field theories can not be formulated in its framework.

The new operator links $v_{2 \mathrm{~N}}=\left|\begin{array}{ll}\mathrm{I}_{\mathrm{N}} & 0 \\ 0 & \hat{C} \mathrm{I}_{\mathrm{N}}\end{array}\right|, \mathrm{N}=$ $2 s+1$, found here between the RCQM of arbitrary spin and the canonical (FW type) field theory enabled ones to translate the result found in these models from one model to another. For example, the results of [27-30] from RCQM can be traslated into the canonical field theory. Contrary, the results of [31] from canonical field theory can be translated into the RCQM (for free non-interacting cases and in the form of anti-Hermitian operators). Note that operator (30) is not unitary but is well defined and having inverse operator.

The partial case of the Schrodinger-Foldy equation, when the wave function has only one component [13], is called the spinless Salpeter equation and is under consideration in many recent papers $[8,29,30,32-37]$. The partial wave packet solutions of this equation are given in [8]. The solutions for free massless and massive particle on a line, massless particle in a linear potential, plane wave solution for a free particle (these solution is given here in (14) for $\mathrm{N}$ component case), free massless particle in three dimensions have been considered. Further, in the paper [34] other time dependent wave packet solutions of the free spinless Salpeter equation are given. Taking into account the relation of such wave packets to the Levy process the spinless Sal- peter equation (in one dimensional space) is called in [34] as the Levy-Schrodinger equation. The several examples of the characteristic behavior of such wave packets have been shown, in particular, of the multimodality arising in their evolutions: a feature at variance with the typical diffusive unimodality of both the corresponding Levy process densities and usual Schrodinger wave functions. Therefore, the interesting task is to extend such consideration to the equations of the $\mathrm{N}$-component relativistic canonical quantum mechanics considered above and to use the links given here in order to transform wave packet solutions $[8,34]$ into the solutions of the equations of the locally covariant field theory.

In this article the original FW transformation [6] is used and slightly generalized for the many component cases. The improvement of the FW transformation [6] is the task of many authors from 1950 until today, see, e. g., the recent publications [115-128]. Nevertheless, this transformation for free case of non-interacting spin $1 / 2$ particle-antiparticle doublet is not changed from 1950 (the year of first publication) until today. Alexander Silenko was successful in FW transformation for single particles with spin 0 [168], spin $1 / 2$ [169] and spin $1[170,171]$ interacting with external electric, magnetic and other fields. In the case of non-interacting particle, when the external electric, magnetic and other external fields are equal to zero, all results of [168-171] and other authors reduce to the earlier results [6, 115]. Therefore, the choice in this paper of the exact FW transformation from 1950 as the initial (and basic for further generalizations for arbitrary spin) is evident and welldefined. In our next articles, we will consider interacting fields and will use the results of [168-171] and recent results of other authors, which generalize the FW formulas in the case of interaction.

A few remarks should be added about the choice of the spin operator. Authors of recent paper [172] considered all spin operators for a Dirac particle satisfying some logical and group-theoretical conditions. The discussion of other spin operators proposed in the literature has been presented as well. As a result only one satisfactory operator has been chosen. This operator is equivalent to the Newton-Wigner spin operator and FW mean-spin operator. Contrary to such way the situation here is evident. Above the choice of the spin operator for spin $s=1 / 2$ particle-antiparticle doublet is unique. The explicit form for such operator follows directly from the main principles of the RCQM of spin $s=1 / 2$ particle-antiparticle doublet, which are formulated in the section 7 of [1]. Such operator from RCQM is given here in formula (39) taken for $\mathrm{N}=2$. After that the links between the RCQM, FW representation and the Dirac model unambiguously give at first the FW spin (40) (with $\mathrm{N}=2$ ) and finally the spin

$$
\vec{s}_{\mathrm{D}}=\vec{s}-\frac{\vec{\gamma} \times \nabla}{2 \widehat{\omega}}+\frac{\nabla \times(\vec{s} \times \nabla)}{\widehat{\omega}(\widehat{\omega}+m)},
$$

which is the FW mean-spin operator (here $\vec{s}$ is given in (40) with $\mathrm{N}=2$ and $\vec{\gamma}$ are $4 \times 4$ standard Dirac matri- 
ces). Therefore, the similar consideration for the higher spin doublets gives unambiguously the well-defined higher spin operators, which are presented in the sections 22-27 of [1]. These new mean-spin operators (259)-(261), (284)(286), (359) in [1] for the $\mathrm{N}$-component Dirac type equations for higher spins $1,3 / 2$ and 2 are the interesting independent results.

The goal of the paper [37] is a comprehensive analysis of the intimate relationship between jump-type stochastic processes (e. g. Levy flights) and nonlocal (due to integrodifferential operators involved) quantum dynamics. Special attention is paid to the spinless Salpeter equation and the various wave packets, in particular to their radial expression in 3D. Furthermore, Foldy's approach [7] is used [37] to encompass free Maxwell theory, which however is devoid of any "particle" content. Links with the photon wave mechanics are explored. Consideration in [1], see e. g. the sections 13, 22 and 23, demonstrates another link between the Maxwell equations and the RCQM. In the generalization of the Foldy's synthesis of covariant particle equations given here (and in [1]) the Maxwell equations and their analogy for nonzero mass are related to the RCQM of spin $\mathrm{s}=(1,1)$ and spin $s=(1,0,1,0)$ particle-antiparticle doublets, another approach see in [173]. The electromagnetic field equations that follow from the corresponding relativistic canonical quantum mechanical equations have been found [174] and in [1]. The new electrodynamical equations containing the hypothetical antiphoton and massless spinless antiboson have been introduced. The Maxwell-like equations for the boson with spin $\mathrm{s}=1$ and $m>0$ (W-boson) have been introduced as well. In other words in [174] (and in [1]), the Maxwell equations for the field with nonzero mass have been introduced.

The resulting system of Maxwell-like equations is given by

$$
\begin{gathered}
\partial_{0} E^{0}+\partial_{j} E^{j} \mp m H^{3}=0, \\
\partial_{0} E^{1}-(\operatorname{curl} \vec{H})^{1}+\partial_{1} E^{0} \mp m E^{2}=0, \\
\partial_{0} E^{2}-(\operatorname{curl} \vec{H})^{2}+\partial_{2} E^{0} \pm m E^{1}=0, \\
\partial_{0} E^{3}-(\operatorname{curl} \vec{H})^{3}+\partial_{3} E^{0} \mp m H^{0}=0, \\
\partial_{0} H^{0}+\partial_{j} H^{j} \pm m E^{3}=0, \\
\partial_{0} H^{1}+(\operatorname{curl} \vec{E})^{1}+\partial_{1} H^{0} \mp m H^{2}=0, \\
\partial_{0} H^{2}+(\operatorname{curl} \vec{E})^{2}+\partial_{2} H^{0} \pm m H^{1}=0, \\
\partial_{0} H^{3}+(\operatorname{curl} \vec{E})^{3}+\partial_{3} H^{0} \pm m E^{0}=0 .
\end{gathered}
$$

We have found the slightly generalized Maxwell equations, known e.g. from [142-144] as massless, with mass.

The system of equations (108) contains 16 equations for the 8 real functions $E^{\alpha}=\left(E^{0}, E^{1}, E^{2}, E^{3}\right), H^{\alpha}=$ $\left(H^{0}, H^{1}, H^{2}, H^{3}\right)$. The situation is similar to the Dirac equation, wherever from the form of 8 equations with $\pm m$

$$
\left(i \gamma^{\mu} \partial_{\mu}-m\right)\left(i \gamma^{\nu} \partial_{\nu}+m\right)=-\partial_{0} \partial_{0}+\Delta-m^{2}
$$

the system of 4 equations with $-m\left(i \gamma^{\mu} \partial_{\mu}-m\right) \psi(x)=0$ for 4 functions is taken. In complete analogy here from the equations (108) the system of 8 equations with upper sign near mass for 8 functions $E^{\alpha}=\left(E^{0}, E^{1}, E^{2}, E^{3}\right), H^{\alpha}=$ $\left(H^{0}, H^{1}, H^{2}, H^{3}\right)$ is taken. It is the system (108) with upper sign near mass.

The system of equations (108) (upper sign near mass) describes the bosonic particle multiplet of the spin $\mathrm{s}=(1,0)$ and mass $m>0$ (the coupled electromagnetic and scalar fields in the case of zero mass). The relation to the Maxwell electrodynamics is evident. In partial case $m=0$ equations (108) (8 real or 4 complex equations) coincide with the Maxwell equations with gradient-like current and charge densities considered in [142-144].

Furthermore, the partial case $E^{0}=H^{0}=0$ in (108) leads to the system of equations

$$
\begin{gathered}
\partial_{j} E^{j}-m H^{3}=0, \\
\partial_{0} E^{1}-(\operatorname{curl} \vec{H})^{1}-m E^{2}=0, \\
\partial_{0} E^{2}-(\operatorname{curl} \vec{H})^{2}+m E^{1}=0, \\
\partial_{0} E^{3}-(\operatorname{curl} \vec{H})^{3}=0, \\
\partial_{j} H^{j}+m E^{3}=0, \\
\partial_{0} H^{1}+(\operatorname{curl} \vec{E})^{1}-m H^{2}=0, \\
\partial_{0} H^{2}+(\operatorname{curl} \vec{E})^{2}+m H^{1}=0, \\
\partial_{0} H^{3}+(\operatorname{curl} \vec{E})^{3}=0 .
\end{gathered}
$$

Equations (110) can be considered as the generalized electrodynamics for the electromagnetic field of the photon with mass. In general, equations (110) describes the field of arbitrary boson with spin $\mathrm{s}=1$ and mass $m>0$.

In [1] the system of equations (110) is shown to be the direct consequence of the equations for the spin $s=(1,1)$ particle-antiparticle doublet.

Moreover, equations (108) with upper sign near mass are linked directly with the RCQM equation for the spin $\mathrm{s}=(1,0)$ multiplet. This link is based on the anti-Hermitian transformations, essential application of complex conjugation operator $\mathrm{C}$ and the corresponding equations for eigenvectors lead to complex number eigenvalues. Therefore, such links and transformations will be given in special publication.

Finally, the substitution $m=0$ into the system of equations (110) leads to the standard Maxwell equations for free electromagnetic field.

The covariant consideration of arbitrary spin field theory given here and in sections 21-28 of [1] contains the noncovariant representations of the Poincaré algebra. Nevertheless, it is not the deficiency of the given model. For the Poincaré group $\mathcal{P}$ generators of spin $\mathrm{s}=(1 / 2,1 / 2)$ the covariant form

$p^{\mu}=i \partial^{\mu}, \quad j^{\mu \nu}=x^{\mu} p^{\nu}-x^{\nu} p^{\mu}+s^{\mu \nu}, \quad s^{\mu \nu} \equiv \frac{i}{4}\left[\gamma^{\mu}, \gamma^{\nu}\right]$, 
is well-known, in which the generators have the form of the local Lie operators. Only in order to have the uniform consideration (82), (83) the Poincaré generators for spin $\mathrm{s}=(1,0,1,0),(3 / 2,3 / 2),(2,0,2,0),(2,1,2,1)$ fields are given in formulae (265), (266), (331), (332), (361), (362) of [1] in corresponding uniform forms of non-covariant operators in covariant theory. Of course, the corrections given here in (85)-(88) for $\vec{s}_{2 \mathrm{~N}}^{\Gamma}$ are necessary. After further transformations of these generators sets in the direction of finding the covariant forms like (111) some sets of generators can be presented in the manifestly covariant forms. For other sets of generators covariant forms are extrinsic. Some sets of generators can be presented only in the forms, which are similar to given in [86-91], where the prime anti-Hermitian operators and specific eigenvectors - eigenvalues equations (with imagine eigenvalues) are used, see, e. g., formula (21) in [88].

The second reason of the stop on the level (265), (266), (331), (332), (361), (362) of [1] is to conserve the important property of the Poincare generators in the canonical FW type representation. Similarly to the FW type Poincaré generators in the sets (265), (266), (331), (332), (361), (362) of [1] both angular momenta (orbital and spin) commute with the operator of the Dirac-like equation of motion (79). Contrary to the generators (82), (83), in the covariant form (111) only total angular momentum, which is the sum of orbital and spin angular momenta, commutes with the $\mathrm{Di}$ racian.

The main point is as follows. The non-covariance is not the barrier for the relativistic invariance! Not a matter of fact that non-covariant objects such as the Lebesgue measure $d^{3} x$ and the non-covariant Poincaré generators are explored, the model of locally covariant field theory of arbitrary spin presented in section 7 is a relativistic invariant in the following sense. The Dirac-like equation (79) and the set $\{\psi\}$ of its solutions (80) are invariant with respect to the reducible representation of the Poincare group $\mathcal{P}$ the nonlocal and non-covariant generators of which are given by (82), (83). Indeed, the direct calculations visualize that generators (82), (83) commute with the operator of equation (79) and satisfy the commutation relations (21) of the Lie algebra of the Poincaré group $\mathcal{P}$.

The partial case of zero mass has been considered briefly in section 28 of [1].

The 8-component manifestly covariant equation (93) for the spin $s=3 / 2$ field found in [1] and here is the $s=3 / 2$ analogy of the 4-component Dirac equation for the spin $s=1 / 2$ doublet. It is shown that synthesis of this equation from the relativistic canonical quantum mechanics of the spin $s=3 / 2$ particle-antiparticle doublet is completely similar to the synthesis of the Dirac equation from the relativistic canonical quantum mechanics of the spin $s=1 / 2$ particleantiparticle doublet. The difference is only in the value of spin $(3 / 2$ and 1/2). On this basis and on the basis of the investigation of solutions and transformation properties with respect to the Poincaré group this new 8-component equation is suggested to be well defined for the description of spin $s=3 / 2$ fermions. Note that known Rarita-Schwinger (Pauli-Fierz) equation has 16 components and needs the additional condition.

The properties of the Fermi-Bose duality, triality and quadro Fermi-Bose properties of equations found have been discussed briefly.

Hence, the method of synthesis of manifestly covariant field equations on the basis of start from the relativistic canonical quantum mechanics of arbitrary spin is suggested. Its approbation on few principal examples is presented.

The main general conclusion is as follows. Among the three main models of arbitrary spin (relativistic canonical quantum mechanics, canonical field theory and covariant field theory) considered here the relativistic canonical quantum mechanics is the best in rigorous quantummechanical description. The transition from the relativistic canonical quantum mechanics to the canonical field theory essentially worsens the quantum-mechanical description. And the final transition from the canonical field theory to the covariant field theory essentially worsens the quantummechanical description once more.

Some results, which are not presented in this smallscale article, can be found in [1]. Indeed, in [1] the following results can be found: the covariant local field theory equations for spin $\mathrm{s}=(1,1)$ particle-antiparticle doublet, spin $\mathrm{s}=(1,0,1,0)$ particle antiparticle multiplet, spin $\mathrm{s}=(3 / 2,3 / 2)$ particle-antiparticle doublet, spin $\mathrm{s}=(2,2)$ particle-antiparticle doublet, spin $s=(2,0,2,0)$ particleantiparticle multiplet and spin $\mathrm{s}=(2,1,2,1)$ particleantiparticle multiplet. The Maxwell-like equations for the boson with spin $\mathrm{s}=1$ and nonzero mass have been introduced in [1] as well. Here these results are given in the general form.

The part of these results, which is related to the spin $\mathrm{s}=1, \mathrm{~s}=(1,1), \mathrm{s}=(1,0,1,0)$ relativistic canonical quantum mechanics and electrodynamics, has been presented in 15-th MMET Conference [174]. Other examples of new equations have been considered in 8-th Symposium on Integrable Systems [175], in XXIII-th and XXIV-th International Conferences on Integrable Systems and Quantum Symmetries in Prague [3, 125].

\section{References}

[1] V.M. Simulik. Covariant local field theory equations following from the relativistic canonical quantum mechanics of arbitrary spin, arXiv: 1409.2766v2 [quant-ph, hep-th] 19 Sep. 2014. 67 p.

[2] V.M. Simulik. Derivation of the Dirac and Dirac-like equations of arbitrary spin from the corresponding relativistic canonical quantum mechanics, Ukr. J. Phys, Vol.60, No.10, 985-1006 (2015).

[3] V.M. Simulik. Link between the relativistic canonical quantum mechanics of arbitrary spin and the corresponding 
field theory, J. Phys: Conf. Ser., Vol.670, 012047(1-16) (2016).

[4] P.A.M. Dirac. The quantum theory of the electron, Proc. R. Soc. Lond. A., Vol.117, No.778, 610-624 (1928).

[5] P.A.M. Dirac. The principles of quantum mechanics, 4-th edition, Clarendon Press, Oxford, 1958, 314 p.

[6] L.L. Foldy, S.A. Wouthuysen. On the Dirac theory of spin $1 / 2$ particles and its non-relativistic limit, Phys. Rev., Vol.78, No.1, 29-36 (1950).

[7] L.L. Foldy. Synthesis of covariant particle equations, Phys. Rev., Vol.102, No.2, 568-581 (1956).

[8] K. Kowalski, J. Rembielinski. Salpeter equation and probability current in the relativistic Hamiltonian quantum mechanics, Phys. Rev. A., Vol.84, No.1, 012108 (1-11) (2011).

[9] V. Simulik, I. Krivsky, I. Lamer. Lagrangian for the spinor field in the Foldy-Wouthuysen representation, Int. J. Theor. Math. Phys., Vol.3, No.4, 109-116 (2013).

[10] R.W. Brown, L.M. Krauss, P.L. Taylor. Leslie Lawrance Foldy, Physics Today, Vol.54, No.12, 75-76 (2001).

[11] V.M. Simulik, I.Yu. Krivsky. Quantum-mechanical description of the fermionic doublet and its link with the Dirac equation, Ukr. J. Phys., Vol.58, No.12, 1192-1203 (2013).

[12] V.M. Simulik, I.Yu. Krivsky. Link between the relativistic canonical quantum mechanics and the Dirac equation, Univ. J. Phys. Appl., Vol.2, No.2, 115-128 (2014).

[13] E.E. Salpeter. Mass corrections to the fine structure of hydrogen-like atoms, Phys. Rev., Vol.87, No.2, 328-343 (1952).

[14] L.L. Foldy. Relativistic particle systems with interaction, Phys. Rev., Vol.122, No.1, 275-288 (1961).

[15] R.A. Weder. Spectral properties of one-body relativistic spin-zero Hamiltonians, Annal. de l'I. H.P., section A., Vol.20, No.2, 211-220 (1974).

[16] R.A. Weder. Spectral analysis of pseudodifferential operators, J. Function. Analys., Vol.20, No.4, 319-337 (1975).

[17] I.W. Herbst. Spectral theory of the operator $\left(\mathbf{p}^{2}+m^{2}\right)^{1 / 2}-Z e^{2} / r$, Comm. Math. Phys., Vol.53, No.3, 285-294 (1977).
[18] I.W. Herbst. Addendum, Comm. Math. Phys., Vol.55, No.3, 316 (1977).

[19] P. Castorina, P. Cea, G. Nardulli, G. Paiano. Partial-wave analysis of a relativistic Coulomb problem, Phys. Rev. D., Vol.29, No.11, 2660-2662 (1984).

[20] L.J. Nickisch, L. Durand. Salpeter equation in position space: Numerical solution for arbitrary confining potentials, Phys. Rev. D., Vol.30, No.3, 660-670 (1984).

[21] A. Martin, S.M. Roy. Semi-relativistic stability and critical mass of a system of spinless bosons in gravitational interaction, Phys. Lett. B., Vol.223, No.3,4, 407-411 (1989).

[22] J.C. Raynal, S.M. Roy, V. Singh, A. Martin, J. Stubble. The ${ }_{i}{ }_{\text {Herbst Hamiltonian }} i \dot{i}$ and the mass of boson stars, Phys. Lett. B., Vol.320, No.1,2, 105-109 (1994).

[23] L.P. Fulcher. Matrix representation of the nonlocal kinetic energy operator, the spinless Salpeter equation and the Cornell potential, Phys. Rev. D., Vol.50, No.1, 447-453 (1994).

[24] J.W. Norbury, K.M. Maung, D.E. Kahana. Exact numerical solution of the spinless Salpeter equation for the Coulomb potential in momentum space, Phys. Rev. A., Vol.50, No.5, 3609-3613 (1994).

[25] W. Lucha, F.F. Schobert. Relativistic Coulomb problem: analytic upper bounds on energy levels, Phys. Rev. A., Vol.54, No.5, 3790-3794 (1996).

[26] W. Lucha, F.F. Schobert. Spinless Salpeter equation: Laguerre bounds on energy levels, Phys. Rev. A., Vol.56, No.1, 139-145 (1997).

[27] F. Brau. Integral equation formulation of the spinless Salpeter equation, J. Math. Phys., Vol.39, No.4, 2254-2263 (1998).

[28] F. Brau. Analytical solution of the relativistic Coulomb problem with a hard core interaction for a one-dimensional spinless Salpeter equation, J. Math. Phys., Vol.40, No.3, 1119-1126 (1999).

[29] F. Brau. Upper limit on the critical strength of central potentials in relativistic quantum mechanics, J. Math. Phys., Vol.46, No.3, 032305 (1-9) (2005).

[30] F. Brau. Lower bounds for the spinless Salpeter equation, J. Nonlin. Math. Phys., Vol.12, suppl. 1, 86-96 (2005). 
[31] T.L. Gill, W.W. Zahary. Analytic representation of the square-root operator, J. Phys. A., Vol.38, No.11, 2479-2496 (2005).

[32] Y. Chargui, L. Chetouani, A. Trabelsi. Analytical treatment of the one-dimensional Coulomb problem for the spinless Salpeter equation, J. Phys. A., Vol.42, No.35, 355203 (1-10) (2009).

[33] Y. Chargui, A. Trabelsi, L. Chetouani. The one-dimensional spinless Salpeter Coulomb problem with minimal length, Phys. Lett. A., Vol.374, No.22, 2243-2247 (2010).

[34] N.C. Petroni. Lévy-Schrödinger wave packets, J. Phys. A., Vol.44, No.16, 165305 (1-27) (2011).

[35] C. Semay. An upper bound for asymmetrical spinless Salpeter equations, Phys. Lett. A., Vol.376, No.33, 2217-2221 (2012).

[36] Y. Chargui, A. Trabelsi. The zero-mass spinless Salpeter equation with a regularized inverse square potential, Phys. Lett. A., Vol.377, No.3-4, 158-166 (2013).

[37] P. Garbaczewski, V. Stephanovich. Lévy flights and nonlocal quantum dynamics, J. Math. Phys., Vol.54, No.7, 072103 (1-34) (2013).

[38] J. von Neumann. Mathematische Grundlagen der Quantenmechanik, Verlag von Julius Springer, Berlin, 1932, 262 p.

[39] P.A.M. Dirac. Relativistic wave equations, Proc. R. Soc. Lond. A., Vol.155, No.886, 447-459 (1936).

[40] M. Fierz. Uber die relativistische Theorie kraftefreier Teilchen mit beliebigem Spin, Helv. Phys. Acta, Vol.12, No.1, 3-37 (1939).

[41] M. Fierz, W. Pauli. On relativistic wave equations for particles of arbitrary spin in an electromagnetic field, Proc. R. Soc. Lond. A., Vol.173, No.953, 211-232 (1939).

[42] H.J. Bhabha. Relativistic wave equations for the elementary particles, Rev. Mod. Phys., Vol.17, No.2-3, 200-216 (1945).

[43] V. Bargman, E.P. Wigner. Group theoretical discussion of relativistic wave equations, Proc. Nat Acad. Sci. USA., Vol.34, No.5, 211-223 (1948).

[44] D.L. Weaver, C.L. Hammer, R.H. Good Jr. Description of a particle with arbitrary mass and spin, Phys. Rev., Vol.135, No.18, B241-B248 (1964).
[45] D.L. Pursey. A Foldy-Wouthuysen transformation for particles of arbitrary spin, Nucl. Phys., Vol.53, No.1, 174-176 (1964).

[46] D.L. Pursey. General theory of covariant particle equations, Ann. Phys. (N.Y.), Vol.32, No.1, 157-191 (1965).

[47] P.M. Mathews. Relativistic Schrödinger equations for particles of arbitrary spin, Phys. Rev., Vol.143, No.4, 978-985 (1966).

[48] T.J. Nelson, R.H. Good Jr. Second-quantization process for particles with any spin and with internal symmetry, Rev. Mod. Phys., Vol.40, No.3, 508-522 (1968).

[49] R.F. Guertin. Relativistic Hamiltonian equations for any spin, Ann. Phys. (N.Y.), Vol.88, No.2, 504-553 (1974).

[50] R.A. Kraicik, M.M. Nieto. Bhabha first-order wave equations. VI. Exact, closed-form, Foldy-Wouthuysen transformations and solutions, Phys. Rev. D., Vol.15, No.2, 433-444 (1977).

[51] R. Illge. Massive fields of arbitrary spin in curved spacetimes, Commun. Math. Phys., Vol.158, No.3, 433-457 (1993).

[52] R-K. Loide, I. Ots, R. Saar. Bhabha relativistic wave equations, J. Phys. A., Vol.30, No.11, 4005-4017 (1997).

[53] R. Illge, R. Schimming. Consistent field equations for higher spin on curved spacetime, Annalen der Physik, Vol.8, No.4, 319-329 (1999).

[54] D.M. Gitman, A.L. Shelepin. Fields on the Poincaré group: arbitrary spin description and relativistic wave equations, Int. J. Theor, Phys., Vol.40, No.3, 603-684 (2001).

[55] H. Shi-Zhong, R. Tu-Nan, Wu Ning, Z. Zhi-Peng. Solution to the Rarita-Schwinger equations, Eur. Phys. J. C., Vol.26, No.4, 609-623 (2003).

[56] A. Zecca. Massive field equations of arbitrary spin in Schwarzschild geometry: separation induced by spin-3/2 case, Int. J. Theor, Phys., Vol.45, No.12, 2241-2247 (2006).

[57] W. Rarita, J. Schwinger. On a theory of particles with half-integral spin, Phys. Rev., Vol.60, No.1, 61 (1941).

[58] A.S. Davydov. Wave equations of a particle having spin $3 / 2$ in the absence of a field, J. Exper. Theor. Phys., Vol.13, No.9-10, 313-319 (1943) (in russian). 
[59] K. Johnson, E.C.G. Sudarshan. Inconsistency of the local field theory of charged spin 3/2 particles, Ann. Phys. (N.Y.), Vol.13, No.1, 126-145 (1961).

[60] G. Velo, D. Zwanziger. Propagation and quantization of Rarita-Schwinger waves in an external electromagnetic potential, Phys. Rev., Vol.186, No.5, 1337-1341 (1969).

[61] G. Velo, D. Zwanziger. Noncausality and other defects of interaction Lagrangians for particles with spin one and higher, Phys. Rev., Vol.188, No.5, 2218-2222 (1969).

[62] G. Velo, D. Zwanziger. Fallacy of perturbative methods for higher-spin equations, Lett. Nuov. Cim., Vol.15, No.2, 39-40 (1976).

[63] C.R. Hagen. New inconsistencies in the quantization of spin- $\frac{3}{2}$ fields, Phys. Rev. D., Vol.4, No.8, 2204-2208 (1971).

[64] J.D. Kimel, L.M. Nath. Quantization of the spin- $\frac{3}{2}$ field in the presence of interactions, Phys. Rev. D., Vol.6, No.8, 2132-2144 (1972).

[65] L.P.S. Singh. Noncausal propagation of classical RaritaSchwinger waves, Phys. Rev. D., Vol.10, No.16, 3297-3305 (1977).

[66] J. Prabhakaran, M. Seetharaman, P.M. Mathews. Causality of propagation of spin- $\frac{3}{2}$ fields coupled to spinor and scalar fields, Phys. Rev. D., Vol.12, No.10, 3191-3194 (1975).

[67] M. Kobayashi, A. Shamaly. Minimal electromagnetic coupling for massive spin-two fields, Phys. Rev. D., Vol.17, No.8, 2179-2181 (1978).

[68] A. Aurilia, M. Kobayashi, Y. Takahashi. Remarks on the constraint structure and the quantization of the RaritaSchwinger field, Phys. Rev. D., Vol.22, No.6, 1368-1374 (1980).

[69] A.Z. Capri, R.L. Kobes. Further problems in spin-3/2 field theories, Phys. Rev. D., Vol.22, No.8, 1967-1978 (1980).

[70] C. Sierra. Classical and quantum aspects of fields with secondary constraints, Phys. Rev. D., Vol.26, No.10, 2730-2744 (1982).

[71] T. Darkhosh. Is there a solution to the Rarita-Schwinger wave equation in the presence of an external electromagnetic field?, Phys. Rev. D., Vol.32, No.12, 3251-3256 (1985).
[72] W. Cox. On the Lagrangian and Hamiltonian constraint algorithms for the Rarita- Schwinger field coupled to an external electromagnetic field, J. Phys. A., Vol.22, No.10, 1599-1608 (1989).

[73] T. Pilling. Symmetry of massive Rarita-Schwinger fields, Int. J. Mod. Phys. A, Vol.20, No.13, 2715-2742 (2005).

[74] M. Napsuciale, M. Kirchbach, S. Rodriguez. Spin 3/2 beyond the Rarita-Schwinger frame- work, Eur. Phys. J. A., Vol.29, No.3, 289-306 (2006).

[75] A.E. Kaloshin, V.P. Lomov. Rarita-Schwinger field and multi-component wave equation, Physics of Particles and Nuclei Letters, Vol.8, No.6, 517-520 (2011).

[76] V.M. Redkov, V.V. Kisel, E.M. Ovsiyuk. Particle with spin 2 and anomalous magnetic moment in external electromagnetic and gravitational fields, arXiv: 1109.1715v1 [math-ph] 8 Sep. 2011. 11 p.

[77] V.M. Redkov. Particle with spin $\mathrm{S}=3 / 2$ in Riemannian space-time, arXiv: 1109.3871v1 [math-ph] 18 Sep. 2011. $12 \mathrm{p}$.

[78] H.J. Bhabha. On a class of relativistic wave equations of spin 3/2, Proc. Indian Acad. Sci. A., Vol.34, No.6, 335-354 (1951).

[79] V.S. Vladimirov. Methods of the theory of generalized functions, Taylor and Francis, London, 2002, 311 p.

[80] N.N. Bogoliubov, A.A. Logunov, I.T. Todorov. Introduction to axiomatic quantum field theory, W.A. Benjamin, Inc., Florida, 1975, 707 p.

[81] P. Garbaczewski. Boson-fermion duality in four dimensions: comments on the paper of Luther and Schotte, Int. J. Theor. Phys., Vol.25, No.11, 1193-1208 (1986).

[82] W.I. Fushchich, A.G. Nikitin. Symmetries of equations of quantum mechanics, Allerton Press Inc., New York, 1994, $480 \mathrm{p}$.

[83] P. Lounesto. Clifford algebras and spinors, 2-nd edition, Cambridge University Press, Cambridge, 2001, 338 p.

[84] V.M. Simulik, I.Yu. Krivsky. Clifford algebra in classical electrodynamical hydrogen atom model, Adv. Appl. Cliff. Algebras, Vol.7, No.1, 25-34 (1997). 
[85] V.M. Simulik, I.Yu. Krivsky. Extended real Clifford-Dirac algebra and bosonic symmetries of the Dirac equation with nonzero mass, arXiv: 0908.3106 [math-ph] 21 Aug. 2009. $5 \mathrm{p}$.

[86] V.M. Simulik, I.Yu. Krivsky. On the extended real CliffordDirac algebra and new physically meaningful symmetries of the Dirac equation with nonzero mass, Reports of the National Academy of Sciences of Ukraine, No.5, 82-88 (2010) (in ukrainian).

[87] I.Yu. Krivsky, V.M. Simulik. Fermi-Bose duality of the Dirac equation and extended real Clifford-Dirac algebra, Cond. Matt. Phys, Vol.13, No.4, 43101 (1-15) (2010).

[88] V.M. Simulik, I.Yu. Krivsky. Bosonic symmetries of the Dirac equation, Phys. Lett. A., Vol.375, No.25, 2479-2483 (2011).

[89] V.M. Simulik, I.Yu. Krivsky, I.L. Lamer. Some statistical aspects of the spinor field Fermi-Bose duality, Cond. Matt. Phys., Vol.15, No.4, 43101 (1-10) (2012).

[90] V.M. Simulik, I.Yu. Krivsky, I.L. Lamer. Application of the generalized Clifford-Dirac algebra to the proof of the Dirac equation Fermi-Bose duality, TWMS J. App. Eng. Math., Vol.3, No.1, 46-61 (2013).

[91] V.M. Simulik, I.Yu. Krivsky, I.L. Lamer. Bosonic symmetries, solutions and conservation laws for the Dirac equation with nonzero mass, Ukr. J. Phys., Vol.58, No.6, 523-533 (2013).

[92] J. Elliott, P. Dawber. Symmetry in Physics, vol.1, Macmillian Press, London, 1979, 366 p.

[93] B. Wybourne. Classical groups for physicists, John Wiley and sons, New York, 1974, 415 p.

[94] K. Jones-Smith, H. Mathur. Relativistic non-Hermitian quantum mechanics, Phys. Rev. D., Vol.89, No.12, 125014(1-5) (2014).

[95] J. Alexandre, C.M. Bender. Foldy-Wouthuysen transformation for non-Hermitian Hamiltonians, J. Phys. A., Vol.48, No.18, 185403(1-8) (2015).

[96] W. Pauli. On the conservation of the lepton charge, Nuov. Cim., Vol.6, No.1, 204-215 (1957).

[97] F. Gürsey. Relation of charge independence and baryon conservation to Pauli's transformation, Nuov. Cim., Vol.7, No.3, 411-415 (1958).
[98] N. Kh. Ibragimov. Invariant variational problems and conservation laws (remarks on Noether's theorem), Theor. Math. Phys., Vol.1, No.3, 267-274 (1969).

[99] W.A. Hepner. The inhomogeneous Lorentz group and the conformal group. $j_{z}$-conserving coupled states approximation, Nuov. Cim., Vol.26, No.2, 351-368 (1962).

[100] M. Petras. The $\mathrm{SO}(3,3)$ group as a common basis for Dirac's and Proca's equations, Nuov. Cim., Vol.46, No.6, 455-464 (1995).

[101] V. Fock. Zur Theorie des Wasserstoffatoms, Z. Phys., Vol.98, No.3-4, 145-154 (1935).

[102] V. Bargmann. Zur Theorie des Wassorstoffatoms. Bemerkungen zur gleichnamigen Arbeit yon V. Fock., Z. Phys., Vol.99, No.7-8, 576-582 (1936).

[103] L.C. Biedenharn. Remarks on the relativistic Kepler problem, Phys. Rev., Vol.126, No.2, 845-851 (1962).

[104] E.H. de Groot. The virial theorem and the Dirac H atom, Am. J. Phys., Vol.50, No.12, 1141-1143 (1982).

[105] A.A. Stahlhofen. Algebraic solutions of relativistic Coulomb problems, Helv. Phys. Acta, Vol.70, No.3, 372-386 (1997).

[106] J-L. Chen, D-L. Deng, M-G. Hu. SO(4) symmetry in the relativistic hydrogen atom, Phys. Rev. A., Vol.77, No.3, 034102(1-4) (2008).

[107] V.M. Simulik, I.O. Gordievich. On the symmetry of relativistic hydrogen atom and the Foldy-Wouthuysen representation, Programm and abstracts of International young scientists conference IEP-2013, Institute of Electron Physics, Uzhgorod, Ukraine, 20-23 May, 2013, p. 217-218 (in russian).

[108] I.Yu. Krivsky, V.M. Simulik. The Dirac equation and spin 1 representations, a connection with symmetries of the Maxwell equations, Theor. Math. Phys., Vol.90, No.3, 265-276 (1992).

[109] I.Yu. Krivsky, R.R. Lompay, V.M. Simulik. Symmetries of the complex Dirac-Kähler equation, Theor. Math. Phys., Vol.143, No.1, 541-558 (2005).

[110] V.M. Simulik. On the old and new matrix representations of the Clifford algebra for the Dirac equation and quantum field theory, World Sci. News, Vol.87, 238-245 (2017). 
[111] D.F. Styer et al. Nine formulations of quantum mechanics, Am. J. Phys., Vol.70, No.3, 288-297 (2002).

[112] B. Thaller. The Dirac equation, Springer, Berlin, 1992, $358 \mathrm{p}$.

[113] N.N. Bogoliubov, D.V. Shirkov. Introduction to the theory of quantized fields, John Wiley and Sons Inc., New York, $1980,620 \mathrm{p}$.

[114] S.I. Kruglov. Dirac-Kähler equation, Int. J. Theor. Phys., Vol.41, No.4, 653-687 (2002).

[115] K.M. Case. Some generalizations of the FoldyWouthuysen transformation, Phys. Rev., Vol.95, No.5, 1323-1328 (1954).

[116] J. Voisin. A construction of the Foldy-Wouthuysen transformation for zero-mass particles of arbitrary spin, Nuov. Cim., Vol.34, No.5, 1257-1262 (1964).

[117] L.M. Garrido, L. Oliver. On the Foldy-Wouthuysen transformation for particles in an electromagnetic field, Nuov. Cim. A., Vol.LII, No.2, 588-605 (1967).

[118] E. de Vries. Foldy-Wouthuysen transformations and related problems, Fortschr. Phys., Vol.18, No.4, 149-182 (1970).

[119] G.A. Ramanujam. On the unitary Foldy-Wouthuysen operator for particles of arbitrary spin, Nuov. Cim. A., Vol.20, No.1, 27-34 (1974).

[120] A.K. Nagpal, R.S. Kaushal. A note on the unitary FoldyWouthuysen operator for arbitrary spin, Lett. Nuov. Cim., Vol.9, No.10, 391-394 (1974).

[121] A.R. Tekumalla, T.S. Santhanam. Unitary FoldyWouthuysen transformations for particles of arbitrary spin, Lett. Nuov. Cim., Vol.10, No.17, 737-740 (1974).

[122] J. Jayaraman. A note on the recent unitary FoldyWouthuysen transformations for particles of arbitrary spin, J. Phys. A., Vol.8, No.1, L1-L4 (1975).

[123] J. Leon, M. Quiros, J.R. Mittelbrunn. Group content of the Foldy-Wouthuysen transformation and the nonrelativistic limit for arbitrary spin, J. Math. Phys., Vol.20, No.6, 1068-1076 (1979).

[124] A.J. Silenko. Exact form of the exponential FoldyWouthuysen transformation operator for an arbitrary-spin particle, Phys. Rev. A, Vol.94, No.3, 032104(1-6) (2016).
[125] V.M. Simulik. Relativistic wave equations of arbitrary spin in quantum mechanics and field theory, example spin $\mathrm{s}=2$, J. Phys: Conf. Ser., Vol.804, 012040(1-10) (2017).

[126] A.D. Bryden. A Foldy-Wouthuysen transformation for particles of spin 3/2, Nucl. Phys., Vol.53, April-May, 165-173 (1964).

[127] J.D. Morrison, R.E. Moss. Approximate solution of the Dirac equation using the Foldy-Wouthuysen hamiltonian, Mol. Phys., Vol.41, No.3, 491-507 (1980).

[128] V.P. Neznamov, A.J. Silenko. Foldy-Wouthuysen wave functions and conditions of transformation between Dirac and Foldy-Wouthuysen representations, J. Math. Phys. Vol.50, No.12, 122302(1-15) (2009).

[129] J. Sakurai. Advanced quantum mechanics, AddisonWesley Pub. Co, New York, 1967, 335 p.

[130] L. Ryder. Quantum field theory, 2-nd edit., University Press, Cambridge, 1996, 487 p.

[131] J. Keller. Theory of the electron. A theory of matter from START, Kluwer Academic Publishers, Dordrecht, 2001, $257 \mathrm{p}$.

[132] V. Fock, D. Iwanenko. Uber eine mogliche geometrische Deutung der relativistischen Quantentheorie, Z. Phys., Vol.54, No.11-12, 798-802 (1929).

[133] V. Fock. Geometrsierung der Diracschen Theorie des Elektrons, Z. Phys., Vol.57, No.3-4, 261-277 (1929).

[134] A. Wightman. Dispersion relations and elementary particles, edit. C. De Witt and R. Omnes, Wiley and Sons, New York, 1960, $671 \mathrm{p}$

[135] H. Sallhofer. Elementary derivation of the Dirac equation I, Z. Naturforsch. A., Vol.33, No.11, 1378-1380 (1978).

[136] H. Sallhofer. Elementary derivation of the Dirac equation X, Z. Naturforsch. A., Vol.41, No.3, 468-470 (1986).

[137] V.M. Simulik. Some algebraic properties of MaxwellDirac isomorphism, Z. Naturforsch. A., Vol.49, No.11, 1074-1076 (1994).

[138] V. Christianto. A review of two derivations of MaxwellDirac isomorphism and a few plausible extensions, viXra: 1705.0357v1 [math-ph] 24 May. 2017. 5 p. 
[139] V.M. Simulik. Connection between the symmetry properties of the Dirac and Maxwell equations. Conservation laws, Theor. Math. Phys., Vol.87, No.1, 386-393 (1991).

[140] V.M. Simulik. Solutions of the Maxwell equations describing the spectrum of hydrogen, Ukr. Math. J., Vol.49, No.7, 1075-1088 (1997).

[141] V.M. Simulik, I.Yu. Krivsky. Bosonic symmetries of the massless Dirac equation, Adv. Appl. Cliff. Algebras, Vol.8, No.1, 69-82 (1998).

[142] V.M. Simulik, I.Yu. Krivsky. Slightly generalized Maxwell classical electrodynamics can be applied to inneratomic phenomena, Ann. Fond. L. de Broglie, Vol.27, No.2, 303-328 (2002).

[143] V.M. Simulik, I.Yu. Krivsky. Relationship between the Maxwell and Dirac equations: symmetries, quantization, models of atom, Rep. Math. Phys., Vol.50, No.3, 315-328 (2002).

[144] V.M. Simulik, I.Yu. Krivsky. Classical electrodynamical aspect of the Dirac equation, Electromagnetic Phenomena, Vol.3, No.1(9), 103-114 (2003).

[145] V.M. Simulik. The electron as a system of classical electromagnetic and scalar fields, edit. V.M. Simulik, Apeiron, Montreal, 2005, $282 \mathrm{p}$.

[146] J. Keller. On the electron theory, Adv. Appl. Cliff. Algebras, Vol.7, Special, 3-26 (1997).

[147] F. Gaioli, E. Alvarez. Some remarks about intrinsic parity in Ryder's derivation of the Dirac equation, Am. J. Phys., Vol.63, No.2, 177-178 (1995).

[148] S. Strinivasan, E. Sudarshan. A direct derivation of the Dirac equation via quaternion measures, J. Phys. A., Vol.29, No.16, 5181-5186 (1996).

[149] L. Lerner. Derivation of the Dirac equation from a relativistic representation of spin, Eur. J. Phys., Vol.17, No.4, 172-175 (1996).

[150] T. Kubo, I. Ohba, H. Nitta. A derivation of the Dirac equation in an external field based on the Poisson process, Phys. Lett. A., Vol.286, No.4, 227-230 (2001).

[151] H. Cui. A method for deriving the Dirac equation from the relativistic Newton's second law, arXiv: 0102114v4 [quant-ph] 15 Aug. 2001. 4 p.
[152] Y.J. Ng, H. van Dam. A geometrical derivation of the Dirac equation, Phys. Lett. A., Vol.309, No.5-6, 335-339 (2003).

[153] M. Calerier, L. Nottale. A scale relativistic derivation of the Dirac equation, Electromagnetic Phenomena, Vol.3, No.1(9), 70-80 (2003).

[154] M. Evans. A generally covariant field equation for gravitation and electromagnetism, Found. Phys. Lett., Vol.16, No.4, 369-377 (2003).

[155] M. Evans. Derivation of Dirac's equation from the Evans wave equation, Found. Phys. Lett., Vol.17, No.2, 149-166 (2004).

[156] S. Efthimiades. Physical meaning and derivation of Schrodinger and Dirac equations, arXiv: 0607001v3 [quant-ph] 4 Jul. 2006. 8 p.

[157] E. Santamato, F. de Martini. Derivation of the Dirac equation by conformal differential geometry, Found. Phys, Vol.43, No.5, 631-641 (2013).

[158] G.M. D'Ariano, P. Perinotti. Derivation of the Dirac equation from principles of information processing, Phys. Rev. A., Vol.90, No.6, 062106(1-18) (2014).

[159] H. Bondi. Negative mass in general relativity, Rev. Mod. Phys., Vol.29, No.3, 423-428 (1957).

[160] E. Recami, G. Zino. About new space-time symmetries in relativity and quantum mechanics, Nuovo Cim. A., Vol.33, No.2, 205-215 (1976).

[161] G. Landis. Comments on negative mass propulsion, J. Propulsion and Power, Vol.7, No.2, 304 (1990).

[162] R. Wayne. Symmetry and the order of events in time, a proposed identity of negative mass with antimatter, Turk. J. Phys., Vol.36, No.2, 165-177 (2012).

[163] W. Kuellin et al. Coherent ballistic motion of electrons in a periodic potential, Phys. Rev. Lett., Vol.104, No.14, 146602(1-4) (2010)

[164] V.P. Neznamov. On the theory of interacting fields in the Foldy-Wouthuysen representation, Phys. Part. Nucl., Vol.37, No.1, 86-103 (2006). 
[165] V.M. Simulik. Relativistic canonical quantum mechanics of arbitrary spin, Proc. of the 9-th Internat. Conference "Methods of non-Euclidean geometry in physics and mathematics (BGL-9)", B.I. Stepanov Institute of Physics, Minsk, Belarus, 27-30 November, 2015, p. 396-409.

[166] I.Yu. Krivsky, T.M. Zajac, S. Shpyrko. Extension of the standard CD algebra in the axiomatic approach for spinor field and Fermi-Bose duality, Adv. Appl. Cliff. Algebras, Vol.27, No.2, 1431-1458 (2017).

[167] V.M. Simulik. On the new matrix representations of Clifford algebra and $\mathrm{SO}(8)$ algebra useful in quantum field theory, Abstracts of the 12-th Summer school "Algebra, topology, analysis", Scientific and educational complex of Dragomanov National Pedagogic Institute, Kolochava, Transcarpathian region, Ukraine, 10-23 July, 2017, p. 16-18 (in ukrainian).

[168] A.J. Silenko. Scalar particle in general inertial and gravitational fields and conformal invariance revisited, Phys. Rev. D., Vol.88, No.4, 045004(1-5) (2013).

[169] A.J. Silenko. Foldy-Wouthyusen transformation and semiclassical limit for relativistic particles in strong external fields, Phys. Rev. A., Vol.77, No.1, 012116(1-7) (2008).

[170] A.J. Silenko. Quantum-mechanical description of spin-1 particles with electric dipole moments, Phys. Rev. D., Vol.87, No.7, 073015(1-5) (2013).
[171] A.J. Silenko. High precision description and new properties of a spin-1 particle in a magnetic field, Phys. Rev. D., Vol.89, No.12, 121701(R)(1-6) (2014).

[172] P. Caban, J. Rembielinski, M. Wlodarczyk. Spin operator in the Dirac theory, Phys. Rev. A., Vol.88, No.2, 022119(18) (2013).

[173] I.Yu. Krivsky, V.M. Simulik, T.M. Zajac, I.L. Lamer. Derivation of the Dirac and Maxwell equations from the first principles of relativistic canonical quantum mechanics, Proc. of the 14-th Internat. Conference "Mathematical Methods in Electromagnetic Theory", Institute of Radiophysics and Electronics, Kharkiv, Ukraine, 28-30 August, 2012, p. 201-204.

[174] V.M. Simulik. Electromagnetic field equations, which follow from the relativistic canonical quantum mechanics, Proc. of the 15-th Internat. Conference "Mathematical Methods in Electromagnetic Theory", O. Honchar National University, Dnipropetrovsk, Ukraine, 26-28 August, 2014, p. 9-30.

[175] V.M. Simulik. Relativistic description of the spin $S=3 / 2$ particle-antiparticle doublet interacting with electromagnetic field, Abstracts of the 8-th Symposium on Integrable Systems, Lodz, Poland, 34 July, 2015, p. 23. 